Prepared in cooperation with the U.S. Army Corps of Engineers, The Nature Conservancy, the Real Edwards Conservation and Reclamation District, and the Texas Parks and Wildlife Department

\title{
Streamflow Gain and Loss and Water Quality in the Upper Nueces River Basin, South-Central, Texas, 2008-10
}

Scientific Investigations Report 2012-5181 
Front cover, Frio River at Garner State Park approximately 8 miles upstream from Concan, Texas (June, 2012).

Back cover, Frio River at Garner State Park approximately 8 miles upstream from Concan, Texas (June, 2012). 


\section{Streamflow Gain and Loss and Water Quality in the Upper Nueces River Basin, South-Central Texas, 2008-10}

By J. Ryan Banta, Rebecca B. Lambert, Richard N. Slattery, and Darwin J. Ockerman

Prepared in cooperation with the U.S. Army Corps of Engineers,

The Nature Conservancy, the Real Edwards Conservation and Reclamation District, and the Texas Parks and Wildlife Department

Scientific Investigations Report 2012-5181 


\title{
U.S. Department of the Interior \\ KEN SALAZAR, Secretary \\ U.S. Geological Survey \\ Marcia K. McNutt, Director
}

\section{U.S. Geological Survey, Reston, Virginia: 2012}

\author{
This and other USGS information products are available at http://store.usgs.gov/ \\ U.S. Geological Survey \\ Box 25286, Denver Federal Center \\ Denver, CO 80225 \\ To learn about the USGS and its information products visit http://www.usgs.gov/ \\ 1-888-ASK-USGS
}

\footnotetext{
Any use of trade, product, or firm names is for descriptive purposes only and does not imply endorsement by the U.S. Government.

Although this report is in the public domain, permission must be secured from the individual copyright owners to reproduce any copyrighted materials contained within this report.
}

Suggested citation:

Banta, J.R., Lambert, R.B., Slattery, R.N., and Ockerman, D.J., 2012, Streamflow gain and loss and water quality in the upper Nueces River Basin, south-central Texas, 2008-10: U.S. Geological Survey Scientific Investigations Report 2012-5181, $40 \mathrm{p}$. 


\section{Contents}

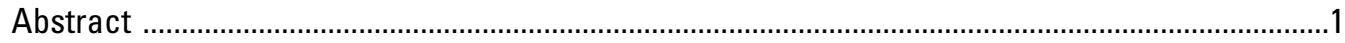

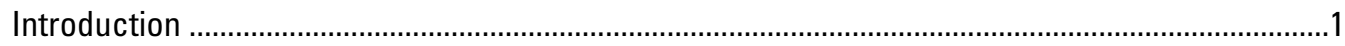

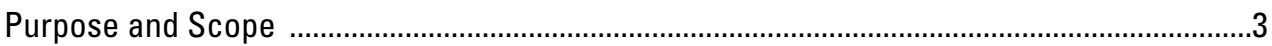

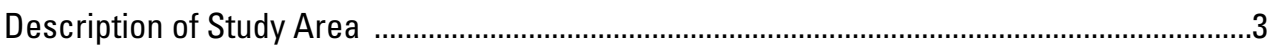

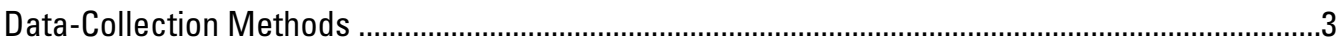

Hydrologic Data Collection ................................................................................................

Quality Assurance of Hydrologic Measurements ..................................................................11

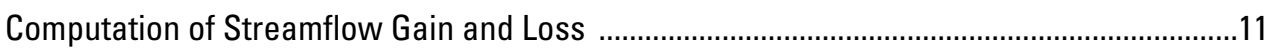

Water-Quality Data Collection .................................................................................................14

Quality Assurance of Water-Quality Samples .....................................................................15

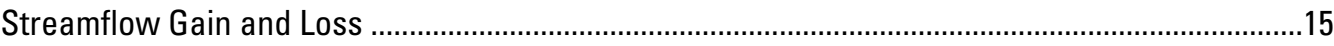

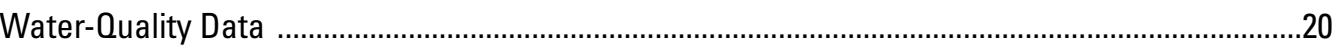

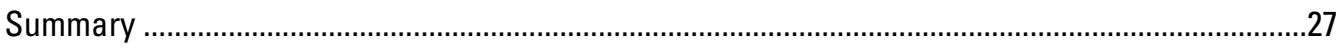

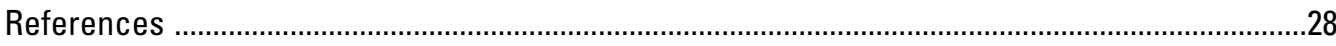

Appendixes 1-3

1. Quality-assurance hydrologic measurements during the July 2008, August 2009, and March 2010 surveys in the upper Nueces River Basin, south-central Texas ..........33

2. Water-quality data collected during the August 2009 and March 2010 surveys in the upper Nueces River Basin, south-central Texas ........................................................34

3. Quality-assurance water-quality data during the August 2009 and March 2010 surveys in the upper Nueces River Basin, south-central Texas

\section{Figures}

1. Map showing location of data-collection sites in the upper Nueces River Basin study area, south-central Texas, 2008-10

2. Map showing Edwards-Trinity (Plateau), Trinity, and Edwards aquifer outcrops and data-collection sites in the upper Nueces River Basin, south-central Texas ..........9

3. Map showing surficial geologic units and data-collection sites in the upper Nueces River Basin, south-central Texas

4. Graphs showing daily mean streamflow at U.S. Geological Survey streamflowgaging stations in south-central Texas during 1950-2010

5. Map showing streamflow measurements in the upper Nueces River Basin, south-central Texas, during July 2008

6. Map showing streamflow and springflow measurements in the upper Nueces River Basin, south-central Texas, during August 2009

7. Map showing streamflow and springflow measurements in the upper Nueces River Basin, south-central Texas, during March 2010

8. Graphs showing comparisons of measured rainfall, streamflow, and groundwater levels in the Nueces River watershed of the upper Nueces River Basin study area, south-central Texas, 2009-10

9. Trilinear diagrams of chemical composition for streamflow, springflow, and groundwater samples collected in the upper Nueces River Basin, south-central Texas, August 2009 
10. Trilinear diagrams of chemical composition for streamflow, springflow, and groundwater samples collected in the upper Nueces River Basin, south-central Texas, March 2010

11. Graphs showing relations of molar ratios to calcium concentrations for streamflow, springflow, and groundwater samples collected in the upper Nueces River Basin, south-central Texas, August 2009 and March 2010

12. Graph showing relation of inverse strontium concentration to strontium-87/ strontium-86 isotopic ratio for streamflow, springflow, and groundwater samples collected in the upper Nueces River Basin, south-central Texas, August 2009 and March 2010

13. Graphs showing relations between molar ratios and strontium-87/strontium-86 isotopic ratios for streamflow, springflow, and groundwater samples collected in the upper Nueces River Basin, south-central Texas, August 2009 and March 2010

\section{Tables}

1. Hydrologic and water-quality data-collection sites and streamflow, springflow, and groundwater measurements in the upper Nueces River Basin, south-central Texas

2. Daily mean streamflow at U.S. Geological Survey streamflow-gaging stations in the upper Nueces River Basin, south-central Texas, during 2008-10 surveys and percentiles of daily mean streamflow from long-term (1950-2010) streamflow records 


\section{Conversion Factors}

\section{Inch/Pound to SI}

\begin{tabular}{lcl}
\hline \multicolumn{1}{c}{ Multiply } & By & \multicolumn{1}{c}{ To obtain } \\
\hline inch (in.) & Length & \\
foot (ft) & 25.4 & millimeter $(\mathrm{mm})$ \\
mile (mi) & 0.3048 & meter $(\mathrm{m})$ \\
& 1.609 & kilometer $(\mathrm{km})$ \\
\hline square mile $\left(\mathrm{mi}^{2}\right)$ & Area & \\
\hline & 2.590 & square kilometer $\left(\mathrm{km}^{2}\right)$ \\
\hline foot per second $(\mathrm{ft} / \mathrm{s})$ & Flow rate & meter per second $(\mathrm{m} / \mathrm{s})$ \\
cubic foot per second $(\mathrm{ft} / \mathrm{s})$ & 0.3048 & cubic meter per second $\left(\mathrm{m}^{3} / \mathrm{s}\right)$ \\
\hline
\end{tabular}

\section{SI to Inch/Pound}

\begin{tabular}{lcl}
\hline \multicolumn{1}{c}{ Multiply } & By & \multicolumn{1}{c}{ To obtain } \\
\hline centimeter $(\mathrm{cm})$ & Length & \\
\hline & 0.3937 & inch (in.) \\
\hline liter $(\mathrm{L})$ & Volume & \\
\hline & 61.02 & cubic inch $\left(\mathrm{in}^{3}\right)$ \\
\hline gram $(\mathrm{g})$ & Mass & \\
kilogram $(\mathrm{kg})$ & 0.03527 & ounce, avoirdupois $(\mathrm{oz})$ \\
\hline
\end{tabular}

Temperature in degrees Celsius $\left({ }^{\circ} \mathrm{C}\right)$ may be converted to degrees Fahrenheit $\left({ }^{\circ} \mathrm{F}\right)$ as follows:

$$
{ }^{\circ} \mathrm{F}=\left(1.8 \times{ }^{\circ} \mathrm{C}\right)+32
$$

Temperature in degrees Fahrenheit $\left({ }^{\circ} \mathrm{F}\right)$ may be converted to degrees Celsius $\left({ }^{\circ} \mathrm{C}\right)$ as follows:

$$
{ }^{\circ} \mathrm{C}=\left({ }^{\circ} \mathrm{F}-32\right) / 1.8
$$

\section{Datums}

Vertical coordinate information is referenced to the North American Vertical Datum of 1988 (NAVD 88).

Horizontal coordinate information is referenced to the North American Datum of 1983 (NAD 83).

\section{Water-Quality Units}

Specific conductance is given in microsiemens per centimeter at 25 degrees Celsius $(\mu \mathrm{S} / \mathrm{cm}$ at $\left.25^{\circ} \mathrm{C}\right)$.

Concentration of chemical constituents in water are given either in milligrams per liter ( $\mathrm{mg} / \mathrm{L}$ ) or in micrograms per liter $(\mu \mathrm{g} / \mathrm{L})$. 


\section{Isotope Units}

Per mil: A unit expressing the ratio of stable-isotope abundances of an element in a sample to those of a standard material. Per mil units are equivalent to parts per thousand. Stable-isotope ratios, also known as delta values, are computed as follows (Kehew, 2001):

$$
\delta X=\left[\left(R_{\text {sample }}-R_{\text {standard }}\right) / R_{\text {standard }}\right] \times 1,000
$$

where

$$
\begin{aligned}
\delta= & \text { delta, } \\
\mathrm{X}= & \text { heavier stable isotope, and } \\
\mathrm{R}= & \text { ratio of rare (heavier) isotope to common (lighter) isotope in sample or } \\
& \text { standard. }
\end{aligned}
$$

The $\delta$ values for stable-isotope ratios discussed in this report are referenced to the following standard materials:

\section{Element}

Hydrogen

Oxygen
$\mathbf{R}$

Hydrogen-2/hydrogen-1

Oxygen-18/oxygen-16

\section{Standard identity and reference}

Vienna Standard Mean Ocean Water (Coplen, 1994)

Vienna Standard Mean Ocean Water (Coplen, 1994) 


\title{
Streamflow Gain and Loss and Water Quality in the Upper Nueces River Basin, South-Central Texas, 2008-10
}

\author{
By J. Ryan Banta, Rebecca B. Lambert, Richard N. Slattery, and Darwin J. Ockerman
}

\section{Abstract}

The U.S. Geological Survey - in cooperation with the U.S. Army Corps of Engineers, The Nature Conservancy, the Real Edwards Conservation and Reclamation District, and the Texas Parks and Wildlife Department - investigated streamflow gain and loss and water quality in the upper Nueces River Basin, south-central Texas, specifically in the watersheds of the West Nueces, Nueces, Dry Frio, Frio, and Sabinal Rivers upstream from the Edwards aquifer outcrop. Streamflow in these rivers is sustained by groundwater contributions (for example, from springs) and storm runoff from rainfall events. To date (2012), there are few data available that describe streamflow and water-quality conditions of the rivers within the upper Nueces River Basin. This report describes streamflow gain-loss characteristics from three reconnaissance-level synoptic measurement surveys (hereinafter referred to as "surveys") during 2008-10 in the upper Nueces River Basin. To help characterize the hydrology, groundwater-level measurements were made, and water-quality samples were collected from both surface-water and groundwater sites in the study area from two surveys during 2009-10. The hydrologic (streamflow, springflow, and groundwater) measurements were made during three reconnaissance-level synoptic measurement surveys occurring in July 21-23, 2008; August 8-18, 2009; and March 22-24, 2010. These survey periods were selected to represent different hydrologic conditions. Streamflow gains and losses were based on streamflow and springflow measurements made at 74 sites in the study area, although not all sites were measured during each survey. Possible water chemistry relations among sample types (streamflow, springflow, or groundwater), between surveys, and among watersheds were examined using water-quality samples collected from as many as 20 sites in the study area.

During the three surveys, reaches of gaining, losing, or no verifiable change in streamflow were observed in the watersheds in the study area. Reaches of generally consistent gaining or losing streamflow were identified in the Nueces, Frio, and Sabinal River watersheds. The water-quality data indicate that the streamflow, springflow, and groundwater have similar major ion chemical characteristics and generally can be categorized as a calcium-carbonate water type. Those data also indicate that the major ion chemistry was similar during the 2009 and 2010 surveys. Graphical comparisons among ratios of major ions, trace elements, and isotopes (for example, magnesium/calcium ratios to strontium isotopic ratios) indicate that samples collected from each watershed generally clustered together. Determining the source areas and other possible contributors on the basis of these data is not possible because of the small sample size of the water-quality dataset (both in number of samples and spatial distribution of samples). The different relations among the water-quality data indicate that the surface water in the different watersheds is likely influenced by differences in source areas, geochemical evolution, groundwater flow paths and residence time, local stratigraphy, or some combination thereof.

\section{Introduction}

The U.S. Geological Survey (USGS) - in cooperation with the U.S. Army Corps of Engineers, The Nature Conservancy, the Real Edwards Conservation and Reclamation District, and the Texas Parks and Wildlife Department - investigated streamflow gain and loss and water quality in the upper Nueces River Basin, south-central Texas, from 2008-10 (fig. 1). The upper Nueces River Basin, defined here as the part of the Nueces River Basin north of the Edwards aquifer outcrop, includes the West Nueces, Nueces, Dry Frio, Frio, and Sabinal Rivers. Streamflow in these rivers is sustained by groundwater contributions (for example, from springs) and storm runoff from rainfall events. The upper Nueces River Basin is an area of particular interest to water managers in south-central Texas because appreciable streamflow gains and losses are observed along various reaches in the area. Gains and losses in the study area have been documented in previous studies (Slade and others, 2002), and anecdotal observations indicate that streamflow might be present at the headwaters of a river, then the river channel may be dry a few miles downstream, and then streamflow reemerges farther downstream. Additionally, rivers originating in the upper Nueces River Basin lose streamflow as they cross the Edwards aquifer outcrop, thus providing recharge to the 


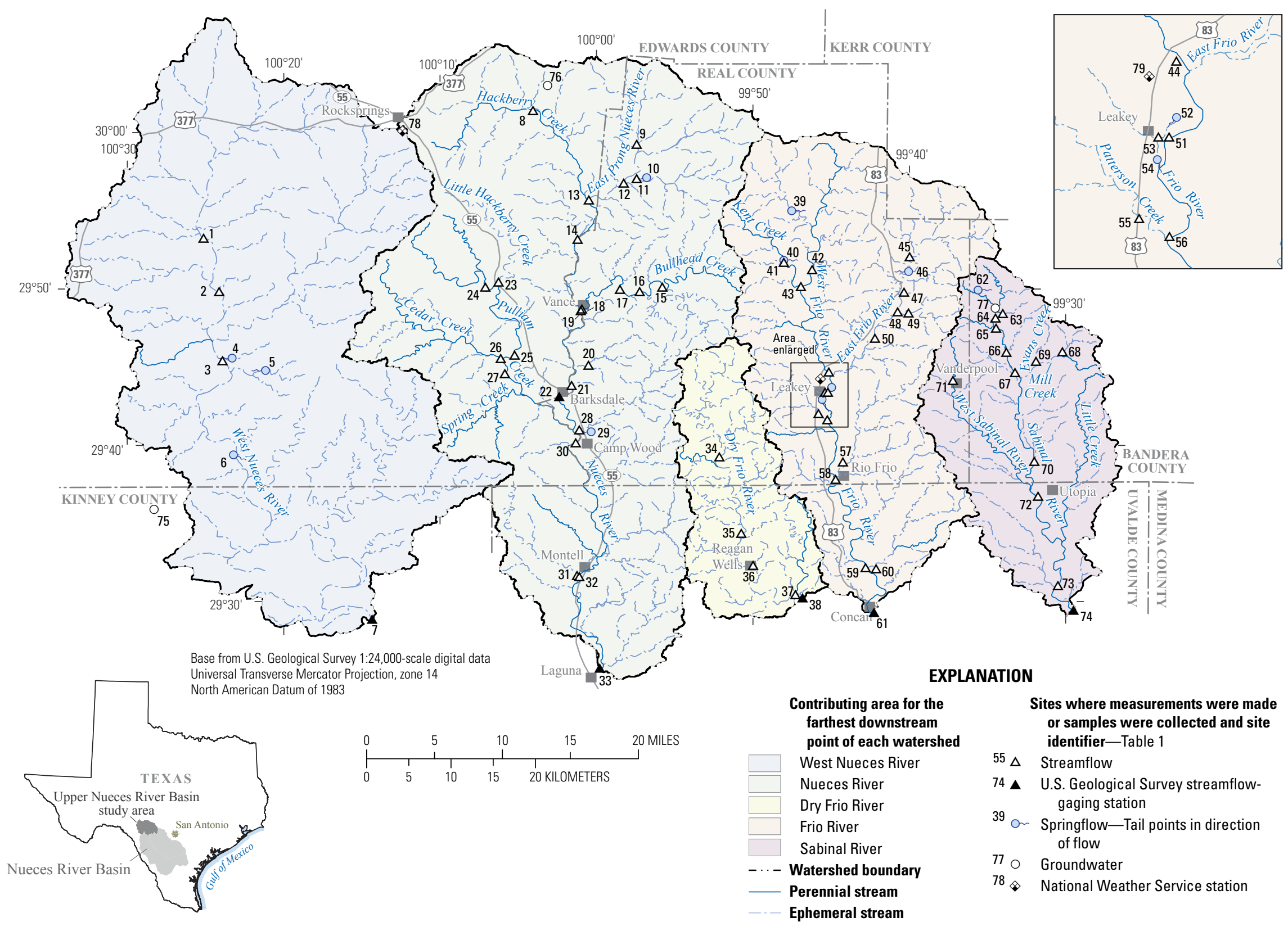

N

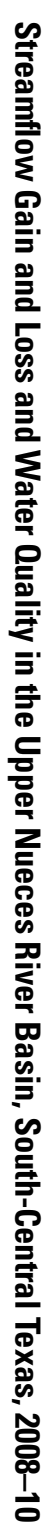

Figure 1. Location of data-collection sites in the upper Nueces River Basin study area, south-central Texas, 2008-10. 
Edwards aquifer (Maclay, 1995). The Edwards aquifer is the primary source of drinking water for the greater San Antonio, Tex., area.

Currently (2012) for the upper Nueces River Basin, few data are available that describe streamflow and water-quality conditions of the West Nueces, Nueces, Dry Frio, Frio, and Sabinal Rivers. Slade and others (2002) identified previous studies of streamflow gains and losses in the upper Nueces River Basin. The earliest streamflow gain-loss synoptic measurement survey was done in 1924 by the Texas Board of Water Engineers (TWBE) (the name and function of TWBE were changed several times by the Texas Legislature, and most functions related to hydrology were transferred to a new agency, the Texas Water Development Board in 1965). Synoptic measurements are defined as "those done concurrently over a broad area at a set time to give a 'snap shot' of hydrologic conditions" (Beck and Wilson, 2006, p. 1). Several streamflow synoptic measurement surveys were done by the Texas Board of Water Engineers in the 1950s, during a period of pronounced drought in central Texas. These 1950s streamflow measurement surveys demonstrated that the upper Nueces River Basin is an area with both streamflow gains and losses (Slade and others, 2002). Since the 1950s, there has been little additional work to measure streamflow gains and losses, and only a few water-quality samples in the study area have been collected for chemical analysis (for example, Fahlquist and Ardis, 2004).

\section{Purpose and Scope}

The purpose of this report is to describe streamflow gainloss characteristics from three reconnaissance-level synoptic measurement surveys in 2008, 2009, and 2010 in the upper Nueces River Basin, south-central Texas. Information obtained from groundwater levels that were measured and water-quality samples that were collected as part of the 2009 and 2010 surveys was used to better understand hydrologic conditions in the study area. Streamflow gains and losses were based on streamflow and springflow measurements made at 74 sites in the study area, although not all sites were measured during each survey. Possible water chemistry relations among sample types (streamflow, springflow, or groundwater), between surveys, and among watersheds were examined using waterquality samples collected from as many as 20 sites in the study area.

\section{Description of Study Area}

The upper Nueces River Basin covers 2,152 square miles in south-central Texas and is composed of the contributing areas for five USGS streamflow-gaging stations (sites 7, 33, 38,61 , and 74 ; fig. 1, table 1). These contributing areas are hereinafter referred to as the West Nueces, Nueces, Dry Frio, Frio, and Sabinal River watersheds, respectively. The study area is predominantly rural and hilly, with an approximate 1,000 -foot ( $\mathrm{ft}$ ) range from the highest to lowest land-surface elevations in the study area (North American Vertical Datum of 1988 [NAVD 88]). The climate of the study area transitions from subtropical steppe in the western part of the study area to subtropical subhumid in the eastern part (Larkin and Bomar, 1983). The rainfall pattern increases from west to east with the average annual rainfall (1951-80) ranging from approximately 22 inches (in.) per year in the western part of the study area to 28 in. per year in the eastern part (Larkin and Bomar, 1983). Daily rainfall amounts greater than 1 in. can occur anytime during the year (Larkin and Bomar, 1983).

The Edwards-Trinity (Plateau) and Trinity aquifers are the primary aquifers in the upper Nueces River Basin area (Ashworth and Hopkins, 1995) (fig. 2). Information regarding surficial geologic formations in the study area was obtained from the "Geologic Atlas of Texas" by the University of Texas, Bureau of Economic Geology: San Antonio Sheet and Del Rio Sheet (Brown, 1963; Brown, 1965; Rose, 1972). The main geologic units outcropping in the study area include the upper member of the Glen Rose Limestone of the Trinity Group, the Devils River Limestone, and the Fort Terrett and Segovia Formations of the Edwards Group (fig. 3). The river channels generally consist of the Fort Terrett Formation of the Edwards Group and the upper member of the Glen Rose Limestone of the Trinity Group in the upper reaches of the study area, and of Quaternary-age deposits (for example, alluvium deposits) in the middle and lower reaches.

\section{Data-Collection Methods}

Hydrologic (streamflow, springflow, and groundwater) measurements were made during three reconnaissance-level synoptic measurement surveys occurring in July 21-23, 2008; August 8-18, 2009; and March 22-24, 2010. Additionally, continuous groundwater levels were measured at site 76 (fig. 1, table 1) during 2009-10. Water-quality samples were collected during the August 2009 and March 2010 surveys.

\section{Hydrologic Data Collection}

In the five watersheds composing the study area (the West Nueces, Nueces, Dry Frio, Frio, and Sabinal Rivers) streamflow or springflow measurements were made at 74 sites, although not all sites were measured during each survey (48 measurement sites during July 2008, 52 measurement sites during August 2009, and 33 measurement sites during March 2010) (fig. 1, table 1). The 74 sites included five USGS streamflow-gaging stations (sites 7, 33, 38, 61, and 74) where streamflow was monitored continuously during the study and which represented the downstream boundary of each of the five watershed drainage areas and at one additional USGS continuous streamflow-gaging station in the Nueces River watershed at site 22 (fig. 1, table 1). The survey measurement locations were selected where access was readily available; for example, considerations of physical constraints and landowner permissions often prevented access to the streams. 
Table 1. Hydrologic and water-quality data-collection sites and streamflow, springflow, and groundwater measurements in the upper Nueces River Basin, south-central Texas.

[dd, degrees; mm, minutes; ss, seconds; ft³/s, cubic foot per second; SW, surface-water streamflow; E, excellent; --, not measured; P, poor; NR, information not released per landowner privacy request; Spring, springflow; QW, water quality; shaded, water-quality sample collected; F, fair; Well, groundwater depth to water; G, good; RF, rainfall]

\begin{tabular}{|c|c|c|c|c|c|c|c|c|c|c|c|c|}
\hline \multirow{2}{*}{$\begin{array}{l}\text { Site } \\
\text { identi- } \\
\text { fier } \\
\text { (fig. 1) }\end{array}$} & \multirow[b]{2}{*}{ Watershed } & \multirow[b]{2}{*}{$\begin{array}{l}\text { U.S. Geological } \\
\text { Survey station } \\
\text { number }\end{array}$} & \multirow[b]{2}{*}{$\begin{array}{l}\text { U.S. Geological } \\
\text { Survey station name }\end{array}$} & \multirow[b]{2}{*}{$\begin{array}{l}\text { Latitude } \\
\text { (dd mm ss) }\end{array}$} & \multirow[b]{2}{*}{$\begin{array}{l}\text { Longitude } \\
\text { (dd mm ss) }\end{array}$} & \multirow[b]{2}{*}{$\begin{array}{l}\text { Type } \\
\text { of data }\end{array}$} & \multicolumn{2}{|c|}{ July 21-23, 2008} & \multicolumn{2}{|c|}{ August 8-18, 2009} & \multicolumn{2}{|c|}{ March 22-24, 2010} \\
\hline & & & & & & & $\begin{array}{l}\text { Measure- } \\
\text { ment } \\
\left(\mathrm{ft}^{3} / \mathrm{s}\right)\end{array}$ & $\begin{array}{l}\text { Measure- } \\
\text { ment } \\
\text { rating }\end{array}$ & $\begin{array}{l}\text { Measure- } \\
\text { ment } \\
\left(\mathrm{ft}^{3} / \mathrm{s}\right)\end{array}$ & $\begin{array}{l}\text { Measure- } \\
\text { ment } \\
\text { rating }\end{array}$ & $\begin{array}{l}\text { Measure- } \\
\text { ment } \\
\left(\mathrm{ft}^{3} / \mathrm{s}\right)\end{array}$ & $\begin{array}{c}\text { Measure- } \\
\text { ment } \\
\text { rating }\end{array}$ \\
\hline 1 & $\begin{array}{l}\text { West Nueces } \\
\text { River }\end{array}$ & 08190100 & $\begin{array}{l}\text { West Nueces River near Rock- } \\
\text { springs, Tex. }\end{array}$ & $29^{\circ} 53^{\prime} 17^{\prime \prime}$ & $100^{\circ} 25^{\prime} 08^{\prime \prime}$ & SW & no flow & $\mathrm{E}$ & -- & -- & -- & -- \\
\hline 2 & $\begin{array}{l}\text { West Nueces } \\
\text { River }\end{array}$ & 08190150 & $\begin{array}{l}\text { West Nueces River at Farm Road } \\
674 \text { near Rocksprings, Tex. }\end{array}$ & $29^{\circ} 49^{\prime} 50^{\prime \prime}$ & $100^{\circ} 24^{\prime} 08^{\prime \prime}$ & SW & no flow & $\mathrm{E}$ & -- & -- & -- & -- \\
\hline 3 & $\begin{array}{l}\text { West Nueces } \\
\text { River }\end{array}$ & 08190195 & $\begin{array}{l}\text { West Nueces River downstream } \\
\text { from Kickapoo Spring near } \\
\text { Rocksprings, Tex. }\end{array}$ & $29^{\circ} 45^{\prime} 24^{\prime \prime}$ & $100^{\circ} 23^{\prime} 55^{\prime \prime}$ & SW & -- & -- & 4.46 & $\mathrm{P}$ & -- & -- \\
\hline 4 & $\begin{array}{l}\text { West Nueces } \\
\text { River }\end{array}$ & 294529100234701 & Spring 1 on West Nueces River ${ }^{1}$ & NR & NR & $\begin{array}{l}\text { Spring, } \\
\text { QW }\end{array}$ & -- & -- & 1.84 & $\mathrm{P}$ & 1.91 & $\mathrm{~F}$ \\
\hline 5 & $\begin{array}{l}\text { West Nueces } \\
\text { River }\end{array}$ & 294443100213701 & Spring 2 on West Nueces River ${ }^{1}$ & NR & NR & $\begin{array}{l}\text { Spring, } \\
\text { QW }\end{array}$ & -- & -- & 0.06 & $P$ & -- & -- \\
\hline 6 & $\begin{array}{l}\text { West Nueces } \\
\text { River }\end{array}$ & 293913100230301 & Spring 3 on West Nueces River ${ }^{1}$ & NR & NR & $\begin{array}{l}\text { Spring, } \\
\text { QW }\end{array}$ & -- & -- & ${ }^{2} 0.01$ & $P$ & -- & -- \\
\hline 7 & $\begin{array}{l}\text { West Nueces } \\
\text { River }\end{array}$ & 08190500 & $\begin{array}{l}\text { West Nueces River near Brackett- } \\
\text { ville, Tex. }\end{array}$ & $29^{\circ} 28^{\prime} 52^{\prime \prime}$ & $100^{\circ} 14^{\prime} 21^{\prime \prime}$ & SW & no flow ${ }^{3}$ & $\mathrm{~F}$ & no flow ${ }^{3}$ & F & ${ }^{3} 0.31$ & $\mathrm{~F}$ \\
\hline 8 & Nueces River & 08189979 & $\begin{array}{l}\text { Hackberry Creek at Farm Road } \\
335 \text { near Vance, Tex. }\end{array}$ & $30^{\circ} 01^{\prime} 25^{\prime \prime}$ & $100^{\circ} 04^{\prime} 04^{\prime \prime}$ & SW, QW & 0.73 & $\mathrm{P}$ & 1.96 & $P$ & 0.19 & $P$ \\
\hline 9 & Nueces River & 0818998070 & $\begin{array}{l}\text { East Prong Nueces River up- } \\
\text { stream of Eagle Ranch near } \\
\text { Rocksprings, Tex. }\end{array}$ & $29^{\circ} 58^{\prime} 59^{\prime \prime}$ & $99^{\circ} 57^{\prime} 24^{\prime \prime}$ & SW, QW & -- & -- & 5.48 & $\mathrm{P}$ & 6.81 & $\mathrm{~F}$ \\
\hline 10 & Nueces River & 295702099571801 & $\begin{array}{l}\text { Spring on East Prong Nueces } \\
\text { River near Vance, Tex. }{ }^{1}\end{array}$ & NR & NR & $\begin{array}{l}\text { Spring, } \\
\text { QW }\end{array}$ & -- & -- & -- & -- & -- & -- \\
\hline 11 & Nueces River & 0818998008 & $\begin{array}{l}\text { Unnamed spring run of East } \\
\text { Prong Nueces River near Rock- } \\
\text { springs, Tex. }\end{array}$ & $29^{\circ} 57^{\prime} 03^{\prime \prime}$ & $99^{\circ} 57^{\prime} 27^{\prime \prime}$ & SW & -- & -- & 1.44 & $\mathrm{P}$ & -- & -- \\
\hline 12 & Nueces River & 08189981 & $\begin{array}{l}\text { East Prong Nueces River down- } \\
\text { stream from East Rose Draw } \\
\text { near Vance, Tex. }\end{array}$ & $29^{\circ} 56^{\prime} 46^{\prime \prime}$ & $99^{\circ} 58^{\prime} 16^{\prime \prime}$ & SW & 11.6 & $P$ & 7.12 & $\mathrm{P}$ & -- & -- \\
\hline 13 & Nueces River & 08189983 & $\begin{array}{l}\text { Nueces River downstream from } \\
\text { Hackberry Creek near Vance, } \\
\text { Tex. }\end{array}$ & $29^{\circ} 55^{\prime} 43^{\prime \prime}$ & $100^{\circ} 00^{\prime} 30^{\prime \prime}$ & SW & 13.3 & $\mathrm{~F}$ & ${ }^{2} 9.63$ & $\mathrm{P}$ & 15.4 & $\mathrm{~F}$ \\
\hline 14 & Nueces River & 08189984 & $\begin{array}{l}\text { Nueces River upstream of Vance, } \\
\text { Tex. }\end{array}$ & $29^{\circ} 53^{\prime} 12^{\prime \prime}$ & $100^{\circ} 01^{\prime} 14^{\prime \prime}$ & SW & 12.4 & $\mathrm{~F}$ & -- & -- & 7.20 & $\mathrm{P}$ \\
\hline
\end{tabular}


Table 1. Hydrologic and water-quality data-collection sites and streamflow, springflow, and groundwater measurements in the upper Nueces River Basin, south-central Texas.-Continued

[dd, degrees; mm, minutes; ss, seconds; $\mathrm{ft}^{3} / \mathrm{s}$, cubic foot per second; SW, surface-water streamflow; E, excellent; --, not measured; P, poor; NR, information not released per landowner privacy request; Spring, springflow; QW, water quality; shaded, water-quality sample collected; F, fair; Well, groundwater depth to water; G, good; RF, rainfall]

\begin{tabular}{|c|c|c|c|c|c|c|c|c|c|c|c|c|}
\hline \multirow{2}{*}{$\begin{array}{l}\text { Site } \\
\text { identi- } \\
\text { fier } \\
\text { (fig. 1) }\end{array}$} & \multirow[b]{2}{*}{ Watershed } & \multirow[b]{2}{*}{$\begin{array}{l}\text { U.S. Geological } \\
\text { Survey station } \\
\text { number }\end{array}$} & \multirow[b]{2}{*}{$\begin{array}{l}\text { U.S. Geological } \\
\text { Survey station name }\end{array}$} & \multirow[b]{2}{*}{$\begin{array}{l}\text { Latitude } \\
\text { (dd mm ss) }\end{array}$} & \multirow[b]{2}{*}{$\begin{array}{l}\text { Longitude } \\
\text { (dd mm ss) }\end{array}$} & \multirow[b]{2}{*}{$\begin{array}{l}\text { Type } \\
\text { of data }\end{array}$} & \multicolumn{2}{|c|}{ July 21-23, 2008} & \multicolumn{2}{|c|}{ August 8-18, 2009} & \multicolumn{2}{|c|}{ March 22-24, 2010} \\
\hline & & & & & & & $\begin{array}{l}\text { Measure- } \\
\text { ment } \\
\left(\mathrm{ft}^{3} / \mathrm{s}\right)\end{array}$ & $\begin{array}{c}\text { Measure- } \\
\text { ment } \\
\text { rating }\end{array}$ & $\begin{array}{l}\text { Measure- } \\
\text { ment } \\
\left(\mathrm{ft}^{3} / \mathrm{s}\right)\end{array}$ & $\begin{array}{c}\text { Measure- } \\
\text { ment } \\
\text { rating }\end{array}$ & $\begin{array}{l}\text { Measure- } \\
\text { ment } \\
\left(\mathrm{ft}^{3} / \mathrm{s}\right)\end{array}$ & $\begin{array}{c}\text { Measure- } \\
\text { ment } \\
\text { rating }\end{array}$ \\
\hline 15 & Nueces River & 08189987 & $\begin{array}{l}\text { Bullhead Creek downstream from } \\
\text { Cord Spring Draw near Vance, } \\
\text { Tex. }\end{array}$ & $29^{\circ} 50^{\prime} 07^{\prime \prime}$ & $99^{\circ} 55^{\prime} 48^{\prime \prime}$ & SW & -- & -- & 0.42 & $\mathrm{P}$ & -- & -- \\
\hline 16 & Nueces River & 0818998770 & $\begin{array}{l}\text { Bullhead Creek at Ranch Road } \\
2631 \text { near Vance, Tex. }\end{array}$ & $29^{\circ} 49^{\prime} 51^{\prime \prime}$ & $99^{\circ} 57^{\prime} 15^{\prime \prime}$ & SW & -- & -- & 0.68 & $\mathrm{P}$ & 0.91 & $\mathrm{P}$ \\
\hline 17 & Nueces River & 08189988 & $\begin{array}{l}\text { Bullhead Creek upstream of } \\
\text { Brushy Draw near Vance, Tex. }\end{array}$ & $29^{\circ} 49^{\prime} 59^{\prime \prime}$ & $99^{\circ} 58^{\prime} 31^{\prime \prime}$ & SW & 1.89 & $\mathrm{P}$ & 1.70 & $\mathrm{P}$ & 4.90 & $\mathrm{P}$ \\
\hline 18 & Nueces River & 08189989 & Bullhead Creek at Vance, Tex. & $29^{\circ} 48^{\prime} 43^{\prime \prime}$ & $100^{\circ} 00^{\prime} 57^{\prime \prime}$ & SW & no flow & $\mathrm{E}$ & 3.79 & $\mathrm{P}$ & -- & -- \\
\hline 19 & Nueces River & 08189985 & Nueces River at Vance, Tex. & $29^{\circ} 48^{\prime} 37^{\prime \prime}$ & $100^{\circ} 01^{\prime} 01^{\prime \prime}$ & SW & 9.12 & $\mathrm{~F}$ & ${ }^{4} 2.19$ & G & -- & -- \\
\hline 20 & Nueces River & 0818998970 & Dry Creek near Barksdale, Tex. & $29^{\circ} 45^{\prime} 08^{\prime \prime}$ & $100^{\circ} 00^{\prime} 31^{\prime \prime}$ & SW & -- & -- & 0.57 & $\mathrm{P}$ & -- & -- \\
\hline 21 & Nueces River & 08189990 & Nueces River at Barksdale, Tex. & $29^{\circ} 43^{\prime} 49^{\prime \prime}$ & $100^{\circ} 01^{\prime} 36^{\prime \prime}$ & SW & 14.8 & $\mathrm{~F}$ & 2.64 & $\mathrm{~F}$ & 36.9 & $\mathrm{~F}$ \\
\hline 22 & Nueces River & 0818999010 & Nueces River near Barksdale, Tex. & $29^{\circ} 43^{\prime} 08^{\prime \prime}$ & $100^{\circ} 02^{\prime} 22^{\prime \prime}$ & SW, QW & -- & -- & ${ }^{3} 16$ & $\mathrm{~F}$ & 350 & $\mathrm{~F}$ \\
\hline 23 & Nueces River & 08189992 & $\begin{array}{l}\text { Little Hackberry Creek at State } \\
\text { Highway } 55 \text { near Barksdale, } \\
\text { Tex. }\end{array}$ & $29^{\circ} 50^{\prime} 27^{\prime \prime}$ & $100^{\circ} 06^{\prime} 17^{\prime \prime}$ & SW & no flow & $\mathrm{P}$ & no flow & $\mathrm{E}$ & -- & -- \\
\hline 24 & Nueces River & 08189991 & $\begin{array}{l}\text { Pulliam Creek upstream of Little } \\
\text { Hackberry Creek near Barks- } \\
\text { dale, Tex. }\end{array}$ & $29^{\circ} 50^{\prime} 06^{\prime \prime}$ & $100^{\circ} 07^{\prime} 07^{\prime \prime}$ & SW & 4.56 & $\mathrm{~F}$ & 1.17 & $\mathrm{~F}$ & -- & -- \\
\hline 25 & Nueces River & 08189993 & $\begin{array}{l}\text { Pulliam Creek upstream of Cedar } \\
\text { Creek near Barksdale, Tex. }\end{array}$ & $29^{\circ} 45^{\prime} 47^{\prime \prime}$ & $100^{\circ} 05^{\prime} 15^{\prime \prime}$ & SW & -- & -- & no flow & $\mathrm{E}$ & 5.48 & $\mathrm{~F}$ \\
\hline 26 & Nueces River & 08189994 & Cedar Creek near Barksdale, Tex. & $29^{\circ} 45^{\prime} 33^{\prime \prime}$ & $100^{\circ} 06^{\prime} 08^{\prime \prime}$ & SW & 0.67 & $\mathrm{P}$ & no flow & $\mathrm{E}$ & 2.70 & $\mathrm{~F}$ \\
\hline 27 & Nueces River & 08189995 & $\begin{array}{l}\text { Pulliam Creek downstream from } \\
\text { Cedar Creek near Barksdale, } \\
\text { Tex. }\end{array}$ & $29^{\circ} 44^{\prime} 36^{\prime \prime}$ & $100^{\circ} 05^{\prime} 52^{\prime \prime}$ & SW & 2.85 & $\mathrm{P}$ & -- & -- & 6.69 & $\mathrm{P}$ \\
\hline 28 & Nueces River & 08189996 & $\begin{array}{l}\text { Camp Wood Creek at State High- } \\
\text { way } 55 \text { near Camp Wood, Tex. }\end{array}$ & $29^{\circ} 41^{\prime} 01^{\prime \prime}$ & $100^{\circ} 01^{\prime} 06^{\prime \prime}$ & SW & no flow & $\mathrm{E}$ & -- & -- & -- & -- \\
\hline 29 & Nueces River & 0818999640 & $\begin{array}{l}\text { Old Faithful Spring near Camp } \\
\text { Wood, Tex. }\end{array}$ & $29^{\circ} 40^{\prime} 46^{\prime \prime}$ & $100^{\circ} 00^{\prime} 48^{\prime \prime}$ & $\begin{array}{l}\text { Spring, } \\
\text { QW }\end{array}$ & -- & -- & -- & -- & 2.49 & $\mathrm{P}$ \\
\hline 30 & Nueces River & 08189997 & Nueces River at Camp Wood, Tex. & $29^{\circ} 40^{\prime} 09^{\prime \prime}$ & $100^{\circ} 01^{\prime} 20^{\prime \prime}$ & SW & 34.7 & $\mathrm{~F}$ & -- & -- & 79.7 & $\mathrm{~F}$ \\
\hline 31 & Nueces River & 0818999850 & Montell Creek near Montell, Tex. & $29^{\circ} 31^{\prime} 41^{\prime \prime}$ & $100^{\circ} 01^{\prime} 15^{\prime \prime}$ & SW & no flow & $\mathrm{E}$ & -- & -- & -- & -- \\
\hline 32 & Nueces River & 08189998 & $\begin{array}{l}\text { Nueces River at County Road } 414 \\
\text { at Montell, Tex. }\end{array}$ & $29^{\circ} 31^{\prime} 35^{\prime \prime}$ & $100^{\circ} 01^{\prime} 06^{\prime \prime}$ & SW & 12.4 & $\mathrm{P}$ & ${ }^{2} 0.71$ & $\mathrm{~F}$ & -- & -- \\
\hline 33 & Nueces River & 08190000 & Nueces River at Laguna, Tex. & $29^{\circ} 25^{\prime} 42^{\prime \prime}$ & $99^{\circ} 59^{\prime} 49^{\prime \prime}$ & SW & ${ }^{3} 31$ & $\mathrm{G}$ & ${ }^{3} 20$ & G & ${ }^{3} 98$ & $\mathrm{G}$ \\
\hline
\end{tabular}


Table 1. Hydrologic and water-quality data-collection sites and streamflow, springflow, and groundwater measurements in the upper Nueces River Basin, south-central Texas.-Continued

[dd, degrees; mm, minutes; ss, seconds; fts/s, cubic foot per second; SW, surface-water streamflow; E, excellent; --, not measured; P, poor; NR, information not released per landowner privacy request; Spring, springflow; QW, water quality; shaded, water-quality sample collected; F, fair; Well, groundwater depth to water; G, good; RF, rainfall]

\begin{tabular}{|c|c|c|c|c|c|c|c|c|c|c|c|c|}
\hline \multirow{2}{*}{$\begin{array}{l}\text { Site } \\
\text { identi- } \\
\text { fier } \\
\text { (fig. 1) }\end{array}$} & \multirow[b]{2}{*}{ Watershed } & \multirow[b]{2}{*}{$\begin{array}{l}\text { U.S. Geological } \\
\text { Survey station } \\
\text { number }\end{array}$} & \multirow[b]{2}{*}{$\begin{array}{l}\text { U.S. Geological } \\
\text { Survey station name }\end{array}$} & \multirow[b]{2}{*}{$\begin{array}{l}\text { Latitude } \\
\text { (dd mm ss) }\end{array}$} & \multirow[b]{2}{*}{$\begin{array}{l}\text { Longitude } \\
\text { (dd mm ss) }\end{array}$} & \multirow[b]{2}{*}{$\begin{array}{l}\text { Type } \\
\text { of data }\end{array}$} & \multicolumn{2}{|c|}{ July 21-23, 2008} & \multicolumn{2}{|c|}{ August 8-18, 2009} & \multicolumn{2}{|c|}{ March 22-24, 2010} \\
\hline & & & & & & & $\begin{array}{l}\text { Measure- } \\
\text { ment } \\
\left(\mathrm{ft}^{3} / \mathrm{s}\right)\end{array}$ & $\begin{array}{c}\text { Measure- } \\
\text { ment } \\
\text { rating }\end{array}$ & $\begin{array}{c}\text { Measure- } \\
\text { ment } \\
\left(\mathrm{ft}^{3} / \mathrm{s}\right)\end{array}$ & $\begin{array}{c}\text { Measure- } \\
\text { ment } \\
\text { rating }\end{array}$ & $\begin{array}{l}\text { Measure- } \\
\text { ment } \\
\left(\mathrm{ft}^{3} / \mathrm{s}\right)\end{array}$ & $\begin{array}{c}\text { Measure- } \\
\text { ment } \\
\text { rating }\end{array}$ \\
\hline 34 & $\begin{array}{c}\text { Dry Frio } \\
\text { River }\end{array}$ & 08195850 & $\begin{array}{l}\text { Dry Frio River upstream of Lee } \\
\text { Creek near Reagan Wells, Tex. }\end{array}$ & $29^{\circ} 39^{\prime} 16^{\prime \prime}$ & $99^{\circ} 52^{\prime} 09^{\prime \prime}$ & SW & -- & -- & no flow & $\mathrm{E}$ & -- & -- \\
\hline 35 & $\begin{array}{l}\text { Dry Frio } \\
\text { River }\end{array}$ & 08195900 & $\begin{array}{l}\text { Dry Frio River downstream from } \\
\text { Lick Creek near Reagan Wells, } \\
\text { Tex. }\end{array}$ & $29^{\circ} 34^{\prime} 22^{\prime \prime}$ & $99^{\circ} 50^{\prime} 45^{\prime \prime}$ & SW & -- & -- & 0.22 & $\mathrm{P}$ & -- & -- \\
\hline 36 & $\begin{array}{l}\text { Dry Frio } \\
\text { River }\end{array}$ & 08195935 & $\begin{array}{l}\text { Dry Frio River upstream of } \\
\text { Brushy Creek at Reagan Wells, } \\
\text { Tex. }\end{array}$ & $29^{\circ} 32^{\prime} 19^{\prime \prime}$ & $99^{\circ} 49^{\prime} 60^{\prime \prime}$ & SW & 0.40 & $\mathrm{P}$ & -- & -- & -- & -- \\
\hline 37 & $\begin{array}{l}\text { Dry Frio } \\
\text { River }\end{array}$ & 08195970 & $\begin{array}{l}\text { Dry Frio River upstream of Fush } \\
\text { Creek near Reagan Wells, Tex. }\end{array}$ & $29^{\circ} 30^{\prime} 27^{\prime \prime}$ & $99^{\circ} 47^{\prime} 17^{\prime \prime}$ & SW & no flow & $\mathrm{E}$ & -- & -- & -- & -- \\
\hline 38 & $\begin{array}{l}\text { Dry Frio } \\
\text { River }\end{array}$ & 08196000 & $\begin{array}{l}\text { Dry Frio River near Reagan } \\
\text { Wells, Tex. }\end{array}$ & $29^{\circ} 30^{\prime} 16^{\prime \prime}$ & $99^{\circ} 46^{\prime} 52^{\prime \prime}$ & SW & ${ }^{3} 1.3$ & $\mathrm{G}$ & ${ }^{3} 0.24$ & G & ${ }^{3} 16$ & $\mathrm{G}$ \\
\hline 39 & Frio River & 295454099473101 & Spring 1 on West Frio River, Tex. ${ }^{1}$ & NR & NR & $\begin{array}{l}\text { Spring, } \\
\text { QW }\end{array}$ & -- & -- & 0.15 & $P$ & -- & -- \\
\hline 40 & Frio River & 295144099480201 & Spring 2 on West Frio River, Tex. ${ }^{1}$ & NR & NR & $\begin{array}{l}\text { Spring, } \\
\text { QW }\end{array}$ & -- & -- & -- & -- & -- & -- \\
\hline 41 & Frio River & 08194695 & $\begin{array}{l}\text { Kent Creek downstream from Ev- } \\
\text { ans Springs near Leakey, Tex. }\end{array}$ & $29^{\circ} 51^{\prime} 43^{\prime \prime}$ & $99^{\circ} 48^{\prime} 02^{\prime \prime}$ & SW & -- & -- & 0.68 & $\mathrm{P}$ & 2.23 & $\mathrm{P}$ \\
\hline 42 & Frio River & 08194690 & $\begin{array}{l}\text { West Frio River upstream of Kent } \\
\text { Creek near Leakey, Tex. }\end{array}$ & $29^{\circ} 51^{\prime} 15^{\prime \prime}$ & $99^{\circ} 46^{\prime} 13^{\prime \prime}$ & SW, QW & 10.4 & $\mathrm{~F}$ & 6.00 & $\mathrm{~F}$ & 7.67 & $P$ \\
\hline 43 & Frio River & 08194700 & $\begin{array}{l}\text { Kent Creek at Farm Road } 336 \\
\text { near Leakey, Tex. }\end{array}$ & $29^{\circ} 50^{\prime} 10^{\prime \prime}$ & $99^{\circ} 46^{\prime} 57^{\prime \prime}$ & SW & 1.34 & $\mathrm{P}$ & 0.45 & $\mathrm{P}$ & 1.94 & $\mathrm{P}$ \\
\hline 44 & Frio River & 08194830 & $\begin{array}{l}\text { West Frio River at U.S. Highway } \\
83 \text { near Leakey, Tex. }\end{array}$ & $29^{\circ} 44^{\prime} 41^{\prime \prime}$ & $99^{\circ} 45^{\prime} 09^{\prime \prime}$ & SW & no flow & $\mathrm{E}$ & -- & -- & 0.50 & $\mathrm{P}$ \\
\hline 45 & Frio River & 08194750 & $\begin{array}{l}\text { East Frio River upstream of Circle } \\
\text { Bluff near Leakey, Tex. }\end{array}$ & $29^{\circ} 52^{\prime} 03^{\prime \prime}$ & $99^{\circ} 39^{\prime} 59^{\prime \prime}$ & SW & -- & -- & 0.58 & $\mathrm{P}$ & -- & -- \\
\hline 46 & Frio River & 295100099403001 & Spring on East Frio River, Tex. ${ }^{1}$ & NR & NR & $\begin{array}{l}\text { Spring, } \\
\text { QW }\end{array}$ & -- & -- & 0.24 & $P$ & -- & -- \\
\hline 47 & Frio River & 08194760 & $\begin{array}{l}\text { Bee Creek at U.S. Highway } 83 \\
\text { near Leaky, Tex. }\end{array}$ & $29^{\circ} 49^{\prime} 48^{\prime \prime}$ & $99^{\circ} 40^{\prime} 21^{\prime \prime}$ & SW & no flow & $\mathrm{E}$ & -- & -- & -- & -- \\
\hline 48 & Frio River & 08194770 & $\begin{array}{l}\text { East Frio River upstream of Cy- } \\
\text { press Creek near Leakey, Tex. }\end{array}$ & $29^{\circ} 48^{\prime} 33^{\prime \prime}$ & $99^{\circ} 40^{\prime} 45^{\prime \prime}$ & SW & 15.2 & $\mathrm{~F}$ & 8.35 & G & -- & -- \\
\hline 49 & Frio River & 08194780 & $\begin{array}{l}\text { Cypress Creek at Cypress Creek } \\
\text { Road near Leakey, Tex. }\end{array}$ & $29^{\circ} 40^{\prime} 29^{\prime \prime}$ & $99^{\circ} 40^{\prime} 06^{\prime \prime}$ & SW & -- & -- & 1.50 & $\mathrm{P}$ & 1.50 & $\mathrm{P}$ \\
\hline
\end{tabular}


Table 1. Hydrologic and water-quality data-collection sites and streamflow, springflow, and groundwater measurements in the upper Nueces River Basin, south-central Texas.-Continued

[dd, degrees; mm, minutes; ss, seconds; $\mathrm{ft}^{3} / \mathrm{s}$, cubic foot per second; SW, surface-water streamflow; E, excellent; --, not measured; P, poor; NR, information not released per landowner privacy request; Spring, springflow; QW, water quality; shaded, water-quality sample collected; F, fair; Well, groundwater depth to water; G, good; RF, rainfall]

\begin{tabular}{|c|c|c|c|c|c|c|c|c|c|c|c|c|}
\hline \multirow{2}{*}{$\begin{array}{c}\text { Site } \\
\text { identi- } \\
\text { fier } \\
\text { (fig. 1) }\end{array}$} & \multirow[b]{2}{*}{ Watershed } & \multirow[b]{2}{*}{$\begin{array}{l}\text { U.S. Geological } \\
\text { Survey station } \\
\text { number }\end{array}$} & \multirow[b]{2}{*}{$\begin{array}{l}\text { U.S. Geological } \\
\text { Survey station name }\end{array}$} & \multirow[b]{2}{*}{$\begin{array}{l}\text { Latitude } \\
\text { (dd mm ss) }\end{array}$} & \multirow[b]{2}{*}{$\begin{array}{l}\text { Longitude } \\
\text { (dd mm ss) }\end{array}$} & \multirow[b]{2}{*}{$\begin{array}{l}\text { Type } \\
\text { of data }\end{array}$} & \multicolumn{2}{|c|}{ July 21-23, 2008} & \multicolumn{2}{|c|}{ August 8-18, 2009} & \multicolumn{2}{|c|}{ March 22-24, 2010} \\
\hline & & & & & & & $\begin{array}{l}\text { Measure- } \\
\text { ment } \\
\left(\mathrm{ft}^{3} / \mathrm{s}\right)\end{array}$ & $\begin{array}{l}\text { Measure- } \\
\text { ment } \\
\text { rating }\end{array}$ & $\begin{array}{l}\text { Measure- } \\
\text { ment } \\
\left(\mathrm{ft}^{3} / \mathrm{s}\right)\end{array}$ & $\begin{array}{l}\text { Measure- } \\
\text { ment } \\
\text { rating }\end{array}$ & $\begin{array}{l}\text { Measure- } \\
\text { ment } \\
\left(\mathrm{ft}^{3} / \mathrm{s}\right)\end{array}$ & $\begin{array}{l}\text { Measure- } \\
\text { ment } \\
\text { rating }\end{array}$ \\
\hline 50 & Frio River & 08194800 & $\begin{array}{l}\text { East Frio River upstream of In- } \\
\text { dian Creek near Leakey, Tex. }\end{array}$ & $29^{\circ} 46^{\prime} 52^{\prime \prime}$ & $99^{\circ} 42^{\prime} 13^{\prime \prime}$ & SW & ${ }^{4} 13.3$ & G & 7.26 & $\mathrm{~F}$ & 18.7 & $\mathrm{~F}$ \\
\hline 51 & Frio River & 08194840 & Frio River at Leakey, Tex. & $29^{\circ} 43^{\prime} 23^{\prime \prime}$ & $99^{\circ} 45^{\prime} 12^{\prime \prime}$ & SW & 4.61 & $\mathrm{P}$ & ${ }^{4} 1.03$ & $\mathrm{~F}$ & 21.7 & $\mathrm{~F}$ \\
\hline 52 & Frio River & 08194845 & Leakey Spring at Leakey, Tex. & $29^{\circ} 43^{\prime} 36^{\prime \prime}$ & $99^{\circ} 45^{\prime} 13^{\prime \prime}$ & Spring & -- & -- & 2.86 & $\mathrm{~F}$ & -- & -- \\
\hline 53 & Frio River & 08194850 & $\begin{array}{l}\text { Leakey Spring Run at Leakey, } \\
\text { Tex. }\end{array}$ & $29^{\circ} 43^{\prime} 23^{\prime \prime}$ & $99^{\circ} 45^{\prime} 24^{\prime \prime}$ & SW, QW & 6.96 & G & 3.61 & G & 9.56 & G \\
\hline 54 & Frio River & 08194860 & Brooks Spring at Leakey, Tex. & $29^{\circ} 42^{\prime} 54^{\prime \prime}$ & $99^{\circ} 45^{\prime} 30^{\prime \prime}$ & Spring & -- & -- & 0.58 & $\mathrm{P}$ & -- & -- \\
\hline 55 & Frio River & 08194900 & $\begin{array}{l}\text { Patterson Creek at U.S. Highway } \\
83 \text { near Leakey, Tex. }\end{array}$ & $29^{\circ} 42^{\prime} 01^{\prime \prime}$ & $99^{\circ} 45^{\prime} 49^{\prime \prime}$ & SW & no flow & $\mathrm{E}$ & -- & -- & 0.40 & $\mathrm{P}$ \\
\hline 56 & Frio River & 08194880 & $\begin{array}{l}\text { Frio River at Farm Road } 1120 \\
\text { downstream from Leakey, Tex. }\end{array}$ & $29^{\circ} 41^{\prime} 39^{\prime \prime}$ & $99^{\circ} 45^{\prime} 15^{\prime \prime}$ & SW & 20.1 & G & -- & -- & -- & -- \\
\hline 57 & Frio River & 08194920 & $\begin{array}{l}\text { Little Dry Frio Creek at Rio Frio, } \\
\text { Tex. }\end{array}$ & $29^{\circ} 38^{\prime} 55^{\prime \prime}$ & $99^{\circ} 44^{\prime} 15^{\prime \prime}$ & SW & no flow & $\mathrm{E}$ & -- & -- & -- & -- \\
\hline 58 & Frio River & 08194930 & Frio River at Rio Frio, Tex. & $29^{\circ} 37^{\prime} 49^{\prime \prime}$ & $99^{\circ} 44^{\prime} 44^{\prime \prime}$ & SW & 10.3 & $\mathrm{~F}$ & -- & -- & -- & -- \\
\hline 59 & Frio River & 08194970 & $\begin{array}{l}\text { Frio River upstream of Concan, } \\
\text { Tex. }\end{array}$ & $29^{\circ} 32^{\prime} 11^{\prime \prime}$ & $99^{\circ} 42^{\prime} 48^{\prime \prime}$ & SW & 19.8 & $\mathrm{~F}$ & -- & -- & -- & -- \\
\hline 60 & Frio River & 08194980 & Bear Creek near Concan, Tex. & $29^{\circ} 32^{\prime} 06^{\prime \prime}$ & $99^{\circ} 42^{\prime} 08^{\prime \prime}$ & SW & no flow & $\mathrm{E}$ & -- & -- & -- & -- \\
\hline 61 & Frio River & 08195000 & Frio River at Concan, Tex. & $29^{\circ} 29^{\prime} 18^{\prime \prime}$ & $99^{\circ} 42^{\prime} 16^{\prime \prime}$ & SW, QW & ${ }^{3} 24$ & G & 37.2 & $\mathrm{G}$ & ${ }^{3} 64$ & G \\
\hline 62 & Sabinal River & 294950099353801 & AS-69-12-401 & $29^{\circ} 49^{\prime} 50^{\prime \prime}$ & $99^{\circ} 35^{\prime} 38^{\prime \prime}$ & $\begin{array}{l}\text { Spring, } \\
\text { QW }\end{array}$ & -- & -- & 0.01 & $\mathrm{P}$ & -- & -- \\
\hline 63 & Sabinal River & 08197905 & $\begin{array}{l}\text { Wedgeworth Creek at Farm Road } \\
187 \text { near Vanderpool, Tex. }\end{array}$ & $29^{\circ} 48^{\prime} 26^{\prime \prime}$ & $99^{\circ} 34^{\prime} 02^{\prime \prime}$ & SW & 3.12 & $\mathrm{P}$ & 1.40 & $\mathrm{P}$ & 1.75 & $\mathrm{P}$ \\
\hline 64 & Sabinal River & 08197907 & $\begin{array}{l}\text { Sabinal River downstream from } \\
\text { Wedgeworth Creek near Van- } \\
\text { derpool, Tex. }\end{array}$ & $29^{\circ} 48^{\prime} 10^{\prime \prime}$ & $99^{\circ} 34^{\prime} 30^{\prime \prime}$ & SW, QW & 1.33 & $\mathrm{P}$ & 1.48 & $\mathrm{~F}$ & 3.37 & $\mathrm{P}$ \\
\hline 65 & Sabinal River & 08197910 & $\begin{array}{l}\text { Sabinal River downstream from } \\
\text { Marler Creek near Vanderpool, } \\
\text { Tex. }\end{array}$ & $29^{\circ} 47^{\prime} 31^{\prime \prime}$ & $99^{\circ} 34^{\prime} 28^{\prime \prime}$ & SW & ${ }^{2} 2.48$ & $\mathrm{~F}$ & 1.25 & $\mathrm{P}$ & -- & -- \\
\hline 66 & Sabinal River & 08197915 & $\begin{array}{l}\text { Brushy Creek near Vanderpool, } \\
\text { Tex. }\end{array}$ & $29^{\circ} 45^{\prime} 58^{\prime \prime}$ & $99^{\circ} 33^{\prime} 48^{\prime \prime}$ & SW & 0.51 & $\mathrm{P}$ & -- & -- & -- & -- \\
\hline 67 & Sabinal River & 08197925 & Sabinal River at Vanderpool, Tex. & $29^{\circ} 44^{\prime} 40^{\prime \prime}$ & $99^{\circ} 33^{\prime} 14^{\prime \prime}$ & SW & 2.65 & $\mathrm{~F}$ & 1.07 & $\mathrm{P}$ & -- & -- \\
\hline 68 & Sabinal River & 08197930 & $\begin{array}{l}\text { Mill Creek at Farm Road } 337 \text { near } \\
\text { Vanderpool, Tex. }\end{array}$ & $29^{\circ} 45^{\prime} 59^{\prime \prime}$ & $99^{\circ} 30^{\prime} 13^{\prime \prime}$ & SW & 0.72 & $\mathrm{P}$ & 0.30 & $\mathrm{P}$ & 2.11 & $\mathrm{~F}$ \\
\hline
\end{tabular}


Table 1. Hydrologic and water-quality data-collection sites and streamflow, springflow, and groundwater measurements in the upper Nueces River Basin, south-central Texas.-Continued

[dd, degrees; mm, minutes; ss, seconds; fts/s, cubic foot per second; SW, surface-water streamflow; E, excellent; --, not measured; P, poor; NR, information not released per landowner privacy request; Spring, springflow; QW, water quality; shaded, water-quality sample collected; F, fair; Well, groundwater depth to water; G, good; RF, rainfall]

\begin{tabular}{|c|c|c|c|c|c|c|c|c|c|c|c|c|}
\hline \multirow{2}{*}{$\begin{array}{l}\text { Site } \\
\text { identi- } \\
\text { fier } \\
\text { (fig. 1) }\end{array}$} & \multirow[b]{2}{*}{ Watershed } & \multirow[b]{2}{*}{$\begin{array}{c}\text { U.S. Geological } \\
\text { Survey station } \\
\text { number }\end{array}$} & \multirow[b]{2}{*}{$\begin{array}{l}\text { U.S. Geological } \\
\text { Survey station name }\end{array}$} & \multirow[b]{2}{*}{$\begin{array}{c}\text { Latitude } \\
\text { (dd mm ss) }\end{array}$} & \multirow[b]{2}{*}{$\begin{array}{l}\text { Longitude } \\
\text { (dd mm ss) }\end{array}$} & \multirow[b]{2}{*}{$\begin{array}{l}\text { Type } \\
\text { of data }\end{array}$} & \multicolumn{2}{|c|}{ July 21-23, 2008} & \multicolumn{2}{|c|}{ August 8-18, 2009} & \multicolumn{2}{|c|}{ March 22-24, 2010} \\
\hline & & & & & & & $\begin{array}{c}\text { Measure- } \\
\text { ment } \\
\left(\mathrm{ft}^{3} / \mathrm{s}\right)\end{array}$ & $\begin{array}{c}\text { Measure- } \\
\text { ment } \\
\text { rating }\end{array}$ & $\begin{array}{c}\text { Measure- } \\
\text { ment } \\
\left(\mathrm{ft}^{3} / \mathrm{s}\right) \\
\end{array}$ & $\begin{array}{c}\text { Measure- } \\
\text { ment } \\
\text { rating }\end{array}$ & $\begin{array}{c}\text { Measure- } \\
\text { ment } \\
\left(\mathrm{ft}^{3} / \mathrm{s}\right)\end{array}$ & $\begin{array}{c}\text { Measure- } \\
\text { ment } \\
\text { rating } \\
\end{array}$ \\
\hline 69 & Sabinal River & 08197933 & $\begin{array}{l}\text { Evans Creek at Farm Road } 337 \\
\text { near Vanderpool, Tex. }\end{array}$ & $29^{\circ} 45^{\prime} 22^{\prime \prime}$ & $99^{\circ} 31^{\prime} 54^{\prime \prime}$ & SW & -- & -- & no flow & $\mathrm{E}$ & -- & -- \\
\hline 70 & Sabinal River & 08197945 & $\begin{array}{l}\text { Sabinal River upstream of Utopia, } \\
\text { Tex. }\end{array}$ & $29^{\circ} 38^{\prime} 59^{\prime \prime}$ & $99^{\circ} 32^{\prime} 01^{\prime \prime}$ & SW & 0.81 & $\mathrm{~F}$ & -- & -- & -- & -- \\
\hline 71 & Sabinal River & 08197955 & $\begin{array}{l}\text { West Sabinal River at Farm Road } \\
337 \text { near Vanderpool, Tex. }\end{array}$ & $29^{\circ} 44^{\prime} 11^{\prime \prime}$ & $99^{\circ} 37^{\prime} 14^{\prime \prime}$ & SW & -- & -- & no flow & $\mathrm{E}$ & 1.72 & $\mathrm{P}$ \\
\hline 72 & Sabinal River & 08197970 & Sabinal River at Utopia, Tex. & $29^{\circ} 36^{\prime} 44^{\prime \prime}$ & $99^{\circ} 31^{\prime} 46^{\prime \prime}$ & SW, QW & no flow & $\mathrm{E}$ & no flow & $\mathrm{E}$ & 23.3 & $P$ \\
\hline 73 & Sabinal River & 08197995 & $\begin{array}{l}\text { Sabinal River upstream of Onion } \\
\text { Creek near Utopia, Tex. }\end{array}$ & $29^{\circ} 31^{\prime} 01^{\prime \prime}$ & $99^{\circ} 30^{\prime} 31^{\prime \prime}$ & SW & 1.17 & $\mathrm{P}$ & 0.12 & $\mathrm{P}$ & -- & -- \\
\hline 74 & Sabinal River & 08198000 & Sabinal River near Sabinal, Tex. & $29^{\circ} 29^{\prime} 27^{\prime \prime}$ & $99^{\circ} 29^{\prime} 33^{\prime \prime}$ & SW & ${ }^{3} 1.4$ & $\mathrm{~F}$ & no flow $^{3}$ & $\mathrm{~F}$ & 337 & $\mathrm{~F}$ \\
\hline
\end{tabular}

\begin{tabular}{|c|c|c|c|c|c|c|c|c|c|c|c|c|}
\hline \multirow[b]{2}{*}{$\begin{array}{l}\text { Site } \\
\text { identi- } \\
\text { fier } \\
\text { (fig. 1) }\end{array}$} & \multirow[b]{2}{*}{ Watershed } & \multirow[b]{2}{*}{$\begin{array}{c}\text { U.S. Geological } \\
\text { Survey station } \\
\text { number }\end{array}$} & \multirow[b]{2}{*}{$\begin{array}{c}\text { State well } \\
\text { number }\end{array}$} & \multirow[b]{2}{*}{$\begin{array}{c}\text { Latitude } \\
\text { (dd mm ss) }\end{array}$} & \multirow[b]{2}{*}{$\begin{array}{l}\text { Longitude } \\
\text { (dd mm ss) }\end{array}$} & \multirow[b]{2}{*}{$\begin{array}{c}\text { Type of } \\
\text { data }\end{array}$} & \multicolumn{2}{|c|}{ July 21-23, 2008} & \multicolumn{2}{|c|}{ August 8-18, 2009} & \multicolumn{2}{|c|}{ March 22-24, 2010} \\
\hline & & & & & & & $\begin{array}{c}\text { Measure- } \\
\text { ment, } \\
\text { depth to } \\
\text { water } \\
\text { (feet) }\end{array}$ & $\begin{array}{c}\text { Measure- } \\
\text { ment } \\
\text { rating }\end{array}$ & $\begin{array}{c}\text { Measure- } \\
\text { ment, } \\
\text { depth to } \\
\text { water } \\
\text { (feet) }\end{array}$ & $\begin{array}{c}\text { Measure- } \\
\text { ment } \\
\text { rating }\end{array}$ & $\begin{array}{c}\text { Measure- } \\
\text { ment, } \\
\text { depth to } \\
\text { water } \\
\text { (feet) }\end{array}$ & $\begin{array}{c}\text { Measure- } \\
\text { ment } \\
\text { rating }\end{array}$ \\
\hline 75 & West Nueces River & 293551100281701 & RP-70-29-101 & $29^{\circ} 35^{\prime} 51^{\prime \prime}$ & $100^{\circ} 28^{\prime} 17^{\prime \prime}$ & Well, QW & -- & -- & 237.37 & -- & 245.34 & -- \\
\hline 76 & Nueces River & 300259100030801 & JJ-55-64-503 & $30^{\circ} 02^{\prime} 59^{\prime \prime}$ & $100^{\circ} 03^{\prime} 08^{\prime \prime}$ & Well, QW & -- & -- & 330.35 & -- & ${ }^{5} 330.49$ & -- \\
\hline 77 & Sabinal River & 294815099343801 & AS-69-12-501 & $29^{\circ} 48^{\prime} 41^{\prime \prime}$ & $99^{\circ} 34^{\prime} 29^{\prime \prime}$ & Well, QW & -- & -- & -- & -- & -- & -- \\
\hline
\end{tabular}

\begin{tabular}{|c|c|c|c|c|c|c|}
\hline $\begin{array}{l}\text { Site } \\
\text { identi- } \\
\text { fier } \\
\text { (fig. 1) }\end{array}$ & Watershed & $\begin{array}{c}\text { National Weather } \\
\text { Service station } \\
\text { number }\end{array}$ & $\begin{array}{c}\text { National Weather } \\
\text { Service station } \\
\text { name }\end{array}$ & $\begin{array}{c}\text { Latitude } \\
\text { (dd mm ss) }\end{array}$ & $\begin{array}{l}\text { Longitude } \\
\text { (dd mm ss) }\end{array}$ & $\begin{array}{c}\text { Type } \\
\text { of data }\end{array}$ \\
\hline 78 & Nueces River & 417706 & Rocksprings 1S & $30^{\circ} 00^{\prime} 11^{\prime \prime}$ & $100^{\circ} 15^{\prime} 36^{\prime \prime}$ & $\mathrm{RF}$ \\
\hline 79 & Frio River & 415113 & Leakey & $29^{\circ} 44^{\prime} 20^{\prime \prime}$ & $99^{\circ} 45^{\prime} 40^{\prime \prime}$ & $\mathrm{RF}$ \\
\hline
\end{tabular}

${ }^{1}$ Official U.S. Geological Survey station name not used here per landowner privacy request.

${ }^{2}$ Average of replicate measurements.

${ }^{3}$ Average of daily mean discharge from continuous measurements during synpotic measurement survey period.

${ }^{4}$ Better rated measurement reported.

${ }^{5}$ Average of daily depth to water (in feet) from continuous measurements during synpotic measurement survey period. 


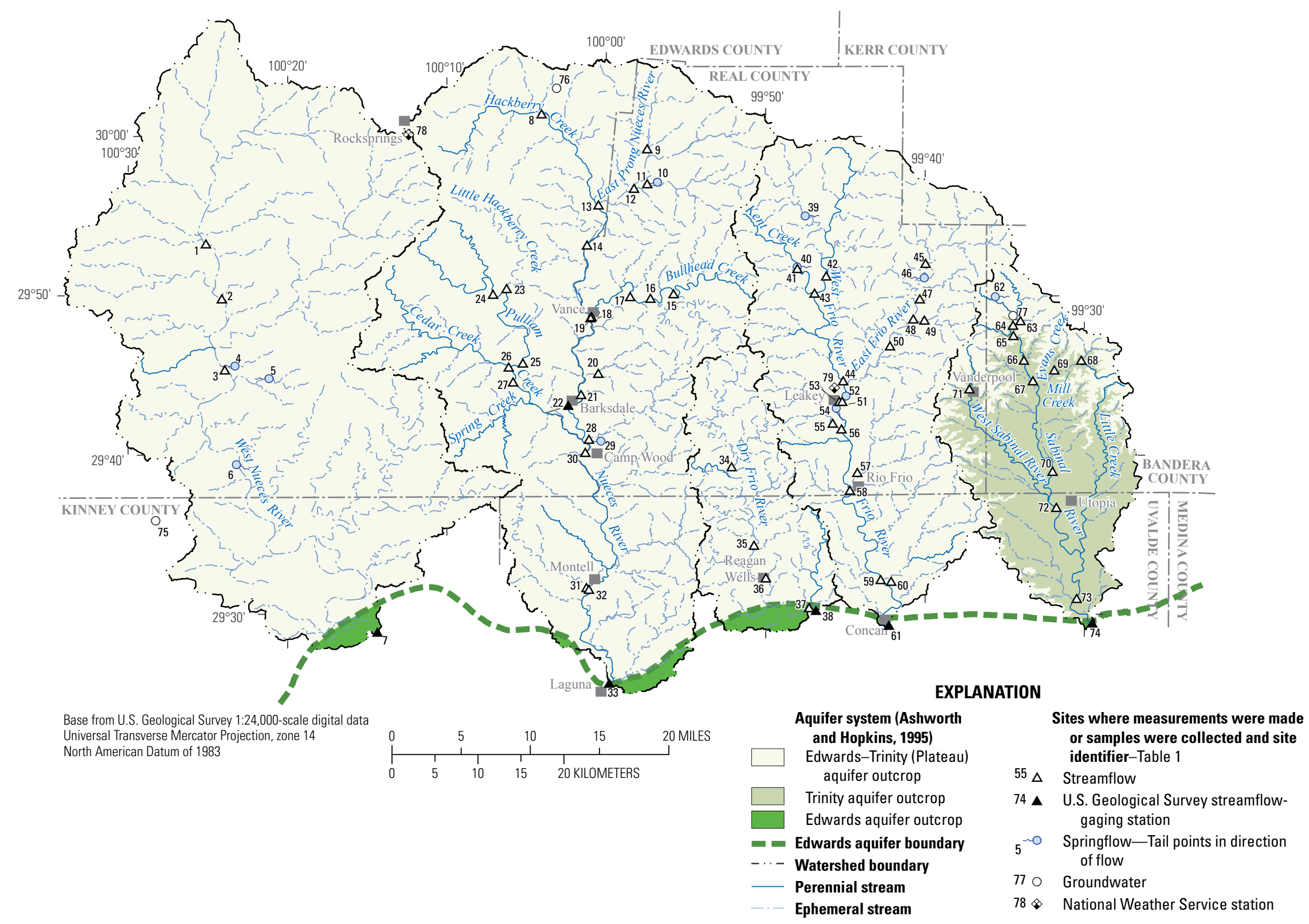

Figure 2. Edwards-Trinity (Plateau), Trinity, and Edwards aquifer outcrops and data-collection sites in the upper Nueces River Basin, south-central Texas. 


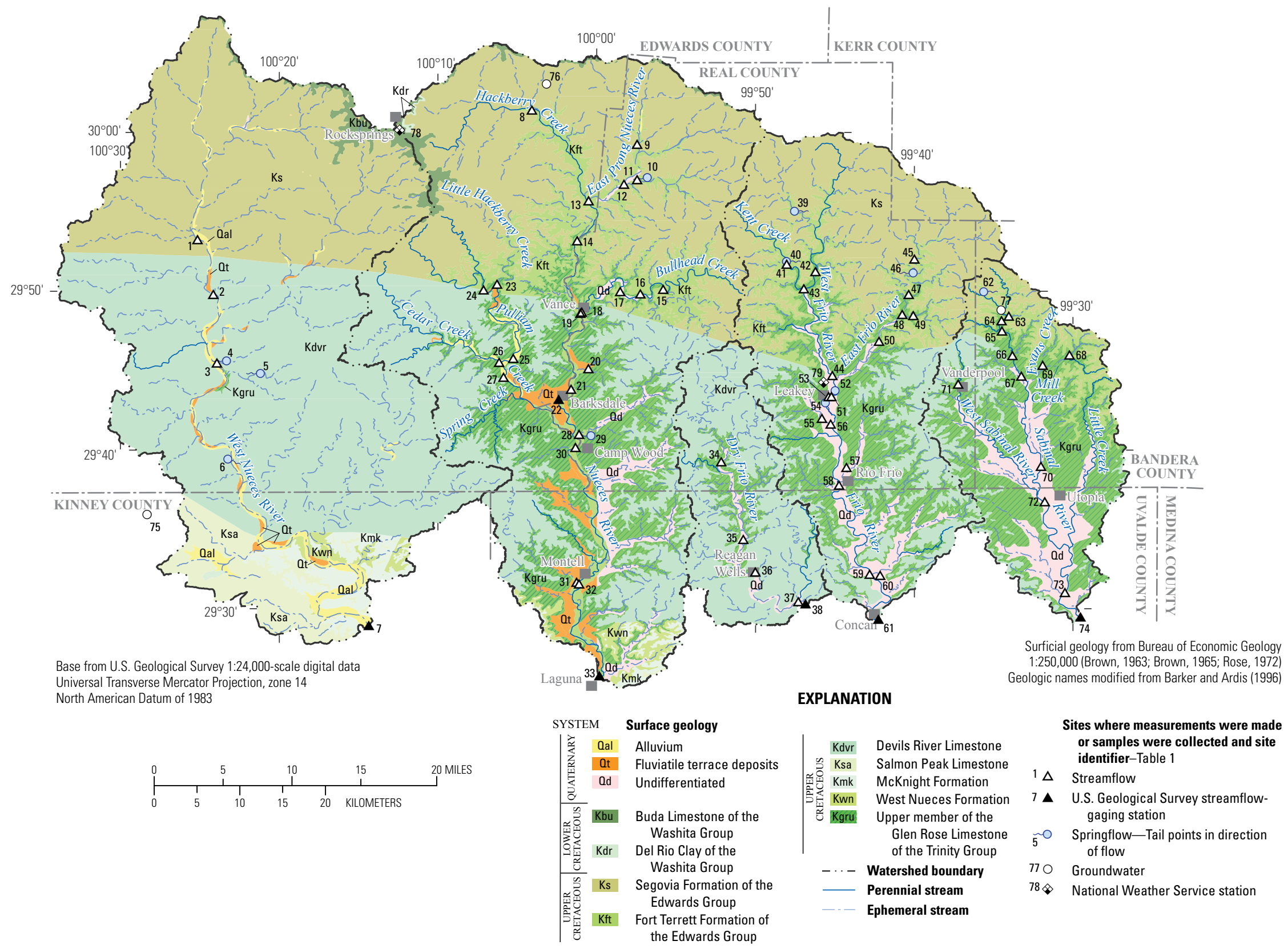

Figure 3. Surficial geologic units and data-collection sites in the upper Nueces River Basin, south-central Texas. 
Streamflow and springflow measurements were made by using USGS methods described by Rantz and others (1982a, b) and Turnipseed and Sauer (2010). The streamflow and springflow measurements were made by wading through the stream and using either a rod-mounted acoustic velocity meter or a pygmy current meter. Some low flows (for example, less than 1 cubic feet per second, $\mathrm{ft}^{3} / \mathrm{s}$ ) were measured by using a portable Parshall flume. Continuous streamflow was measured at the six USGS streamflow-gaging stations in the study area by following methods described by Turnipseed and Sauer (2010) and Rantz and others (1982a, b).

Flow conditions in each of the five watersheds composing the study area were evaluated by using the longterm (1950-2010) streamflow records from the farthest downstream USGS streamflow-gaging station in each of the watersheds (U.S. Geological Survey, 2012a). The surveys were done during conditions chosen to represent three different streamflow conditions: "low-flow," "very low flow," and "medium-flow" conditions during July 2008, August 2009, and March 2010, respectively. Specifically, in July 2008 , when streamflow at the farthest downstream station in each of the five contributing watersheds was generally less than the 25th percentile of long-term streamflow measured at these stations, the streamflow conditions were referred to as "low flow"; "very low flow" refers to conditions that existed in August 2009 when streamflow of the five rivers generally was less than the 10th percentile of the respective long-term streamflows; and "medium flow" refers to conditions that existed in March 2010 when streamflow was generally around the 50th percentile of long-term streamflow. For example, during the 1950-2010 period of record at site 33 (fig. 1), the farthest downstream gage in the Nueces River watershed within the study area, the daily mean streamflow was $31 \mathrm{ft}^{3} / \mathrm{s}$ (low flow), $20 \mathrm{ft}^{3} / \mathrm{s}$ (very low flow), and $98 \mathrm{ft}^{3} / \mathrm{s}$ (medium flow) during the 2008, 2009, and 2010 surveys, respectively (fig. 4, table 2). While the three surveys do not capture the full range of historical streamflows, they do represent a range of different hydrologic conditions.

To better understand the gain-loss characteristics of stream reaches in the study area, each survey was done during periods of stable flow, selected to minimize the possible influence of storm runoff on streamflow. For example, in the 7 days prior to the 2008 and 2009 surveys, no rainfall was measured at Rocksprings, Tex., near the headwaters of Nueces River, and in the 7 days prior to the 2010 survey, a total of only 0.5 in. of rainfall was measured (National Weather Service [NWS] cooperative station number 417706, Rocksprings 1S; National Climatic Data Center, 2011). Rainfall amounts were similar at Leakey, Tex., in the central region of the study area (NWS cooperative station number 415113; National Climatic Data Center, 2011). Because scant rainfall occurred prior to each survey, the effect of potential storm runoff on streamflow conditions was considered minimal.

Groundwater-level measurements were collected at two sites (75-76) by using a graduated electric tape and following methods described by Cunningham and Schalk (2011). Groundwater-level measurements were reported as depth to water (in feet) below the land surface at the well. Continuous groundwater-level measurements were made at site 76 every 15 minutes with a pressure transducer and transmitted every 4 hours by a Geostationary Operational Environmental Satellite (GOES) to the USGS National Water Information System (NWIS) database (U.S. Geological Survey, 2012a). The groundwater-level data were verified and corrected as necessary by periodic measurements using a graduated electric tape, as described by Freeman and others (2004).

\section{Quality Assurance of Hydrologic Measurements}

Quality-assurance measurements (replicate measurements) were made for selected streamflow and springflow measurements. Quality-assurance measurements were compared to the associated primary measurements by calculating the relative percent difference (RPD) (app. 1). The RPD was computed by using the equation

$$
\mathrm{RPD}=\left[\left|\mathrm{X}_{1}-\mathrm{X}_{2}\right|\right] /\left[\left(\mathrm{X}_{1}+\mathrm{X}_{2}\right) / 2\right] \times 100,
$$

where

$$
\begin{aligned}
& \mathrm{X}_{1} \quad \text { = primary measurement for the variable of } \\
& \text { interest, and } \\
& \mathrm{X}_{2} \quad=\text { replicate measurement for the variable of }
\end{aligned}
$$

Generally, the RPDs for streamflow and springflow measurements were within 5 percent. Larger RPDs were measured during low-flow conditions in tributary streams and when springflow amounts were relatively small. For instance, the largest RPD (51 percent) was calculated for two replicate streamflow measurements ( 1.03 and $\left.0.61 \mathrm{ft}^{3} / \mathrm{s}\right)$ made at site 51 (app. 1). The percent difference in this example might be in part because the streamflow measurement was rated fair and the quality assurance measurement was rated poor. Where replicate measurements were made, the measurement with the better quality rating assigned by the hydrographer was used in the analyses and reported in table 1. For example, if measurements were rated good and fair, the good measurement was used for the analyses, or if the measurements were rated the same, the average of the two measurements was reported (table 1) and used in subsequent analyses. At streamflowgaging stations where continuous daily mean streamflows were available, the average of the daily mean streamflows during the survey period was reported.

\section{Computation of Streamflow Gain and Loss}

Stream reaches can be characterized as having a gain, a loss, or no change in streamflow. Possible sources of gains or losses can be attributed to groundwater inflow or outflow, inflow from tributaries and return flows, outflow from diversions, and evaporation (Slade and others, 2002; 

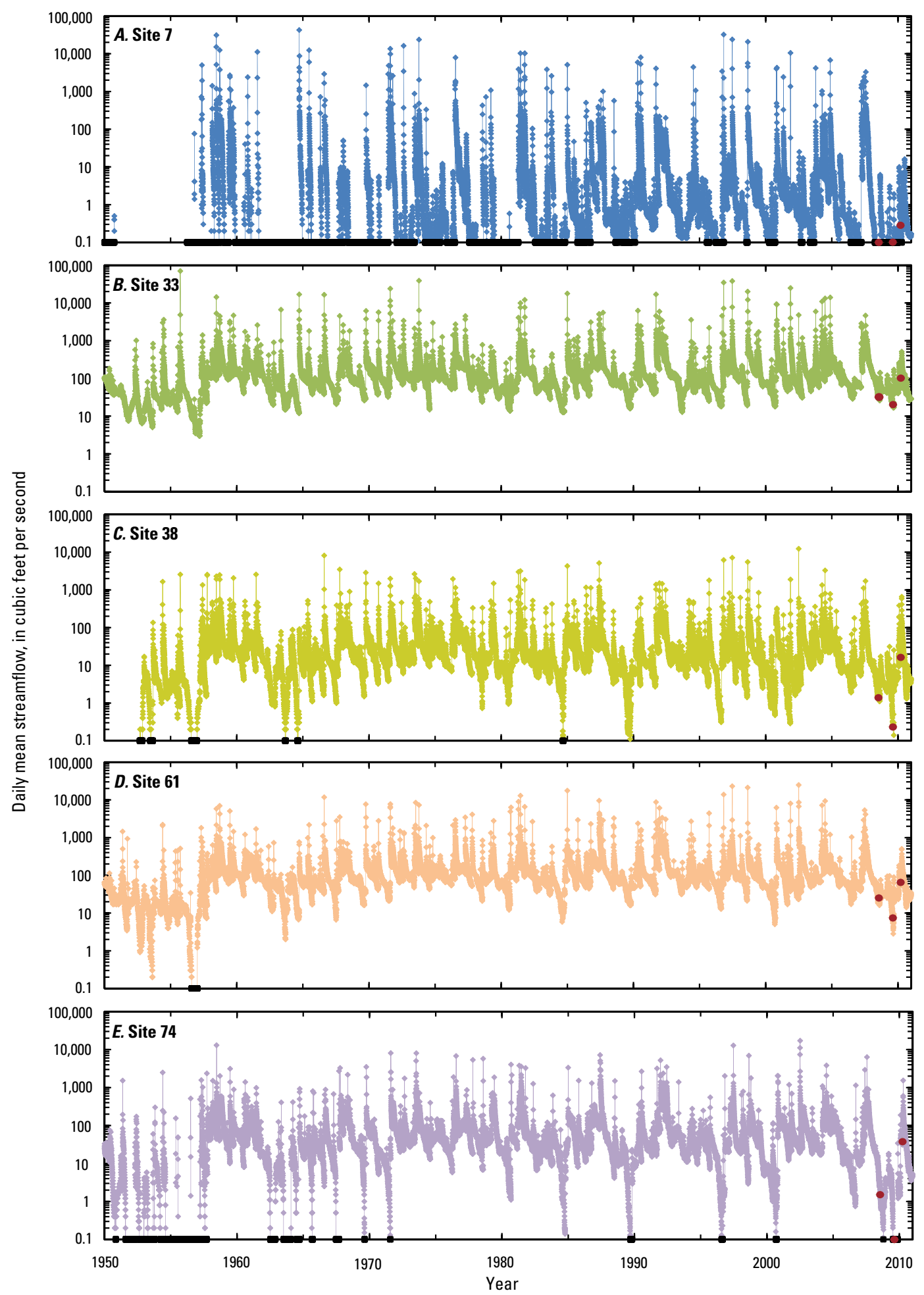

EXPLANATION

- Daily mean streamflow during survey

- Daily mean streamflow less than 0.1 cubic foot per second

Figure 4. Daily mean streamflow at U.S. Geological Survey streamflow-gaging stations in south-central Texas during 1950-2010. A, 08190500 West Nueces River near Brackettville, Tex. (site 7). B, 08190000 Nueces River at Laguna, Tex. (site 33). C, 08196000 Dry Frio River near Reagan Wells, Tex. (site 38). D, 08195000 Frio River at Concan, Tex. (site 61). E, 08198000 Sabinal River near Sabinal, Tex. (site 74). 
Table 2. Daily mean streamflow at U.S. Geological Survey streamflow-gaging stations in the upper Nueces River Basin, south-central Texas, during 2008-10 surveys and percentiles of daily mean streamflow from long-term (1950-2010) streamflow records.

[Data are from U.S. Geological Survey, 2012a]

\begin{tabular}{|c|c|c|c|c|c|}
\hline \multirow[b]{2}{*}{ Survey } & \multicolumn{5}{|c|}{ Daily mean streamflow during 2008-10 surveys } \\
\hline & $\begin{array}{c}\text { U.S. Geological } \\
\text { Survey station } \\
08190500 \\
\text { West Nueces River } \\
\text { near Brackettville, Tex. } \\
\text { (site 7) }\end{array}$ & $\begin{array}{c}\text { U.S. Geological } \\
\text { Survey station } \\
08190000 \\
\text { Nueces River at } \\
\text { Laguna, Tex. } \\
\text { (site 33) }\end{array}$ & $\begin{array}{c}\text { U.S. Geological } \\
\text { Survey station } \\
08196000 \\
\text { Dry Frio River near } \\
\text { Reagan Wells, Tex. } \\
\text { (site 38) }\end{array}$ & $\begin{array}{c}\text { U.S. Geological } \\
\text { Survey station } \\
08195000 \\
\text { Frio River at } \\
\text { Concan, Tex. } \\
\text { (site 61) }\end{array}$ & $\begin{array}{c}\text { U.S. Geological } \\
\text { Survey station } \\
08198000 \\
\text { Sabinal River } \\
\text { near Sabinal, Tex. } \\
\text { (site 74) }\end{array}$ \\
\hline March 22-24, 2010 & 0.31 & 98 & 16 & 64 & 37 \\
\hline
\end{tabular}

\begin{tabular}{|c|c|c|c|c|c|}
\hline \multirow{2}{*}{ Percentile } & \multicolumn{5}{|c|}{ Daily mean streamflow from long-term streamflow records ${ }^{1}$} \\
\hline & Site 7 & Site 33 & Site 38 & Site 61 & Site 74 \\
\hline 10th percentile & no flow & 26 & 2.3 & 18 & no flow \\
\hline 25 th percentile & no flow & 50 & 6.3 & 44 & 8.8 \\
\hline Median & 0.22 & 87 & 14 & 74 & 28 \\
\hline 75th percentile & 1.9 & 156 & 31 & 125 & 63 \\
\hline Maximum & 42,500 & 70,300 & 12,200 & 24,700 & 17,100 \\
\hline
\end{tabular}

${ }^{1}$ Long-term streamflow record for site 38 is $1952-2010$.

Ockerman, 2002; Turco, 2007; Braun, 2011). The methods used for computation of streamflow gain and loss followed those described in Ockerman (2002, p. 7):

Groundwater inflow to the streams or outflow to groundwater are not directly measurable because the inflow and outflow processes usually cannot be observed. Even visible inflow from springs and seeps cannot always be measured accurately. Therefore, inflow from or outflow to groundwater in subreaches of the streams was measured indirectly by measuring the difference in streamflow at the upstream and downstream ends of the subreaches. Groundwater inflow and outflow are not the only sources of gain or loss along the streams. Other sources are tributary inflow, diversions, return flows, and evaporation. By accounting for these factors, groundwater inflow or outflow can be estimated. Groundwater inflow, $G$, is estimated as

$$
\mathrm{G}=\mathrm{Q}_{\mathrm{D}}-\mathrm{Q}_{\mathrm{U}}-\mathrm{I}+\mathrm{D}-\mathrm{R}+\mathrm{E},
$$

where

$$
\begin{aligned}
& \mathrm{Q}_{\mathrm{D}} \quad=\begin{array}{l}
\text { measured streamflow at the downstream } \\
\text { boundary of the subreach, }
\end{array} \\
& \mathrm{Q}_{\mathrm{U}} \quad \begin{array}{l}
\text { measured streamflow at the upstream } \\
\text { boundary of the subreach, }
\end{array}
\end{aligned}
$$

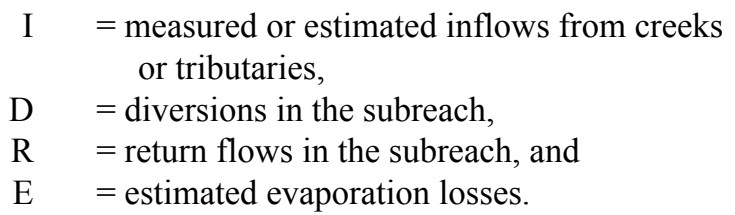

Positive groundwater inflow indicates the reach is gaining streamflow, whereas negative groundwater inflow indicates the reach is losing streamflow. During this study, some tributary inflows were measured when field staff had access to the tributary site, however, there might have been unmeasured contributing inflows in some reaches. For this report, unquantified inflows from tributaries were not estimated. For example, a downstream reach might indicate a gain in streamflow, but this gain could also be a function of an unquantified tributary contributing to the streamflow. These unknown contributions might affect the interpretation of reaches gaining or losing streamflow, but a reach identified as losing streamflow that has a potential unmeasured contributing tributary is still a losing reach, regardless of the magnitude of contribution from the tributary. Streamflow gain-loss was not calculated for tributary reaches where the tributary was not measured at the confluence with the main stem. Although there are several permitted withdrawals in the study area, during the study there was only one reported diversion of streamflow (averaging $1.1 \mathrm{ft}^{3} / \mathrm{s}$ ), and this diversion occurred during the 2009 survey, about 4 miles upstream from site 32 . 
Accounting for this diversion in the calculation of streamflow gain-loss (eq. 2) did not change the resulting classification of "losing" in this reach. No other known diversions occurred during the 2008-10 surveys (Angela Sander, Texas Commission of Environmental Quality, written commun., 2012). There are no known return flows in the study area (for example, effluent from wastewater treatment plants). Evaporation was not measured. As explained by Turco (2007, p. 7), "the magnitude of error associated with [excluding evaporation] from equation 1 is assumed to be minor when compared to the potential errors associated with the flow measurements."

The streamflow gain or loss was then classified as "verifiable" as described in Braun and Lambert (2011, p. 14-15):

A stream reach is classified as verifiably gaining or losing only if the difference in streamflow between the upstream and downstream measuring sites exceeds the potential error associated with the flow measurements made at those locations. Measurement error is a function of the streamflow measurement rating (excellent, good, fair, or poor) as determined by the hydrographer (streamgager) (Sauer and Meyer, 1992). The rating is based on streambed conditions, velocity homogeneity, crosssection uniformity, and any additional factors that affect the measurement accuracy. Measurements rated as excellent are believed to be within 2 percent of the actual flow, good are believed to be within 5 percent of flow, fair are believed to be within 8 percent of flow, and poor are believed to differ from actual flow by greater than 8 percent (set at 10 percent for the purposes of error calculations in this report).

\section{Water-Quality Data Collection}

Water-quality and associated quality-control samples were collected from streamflow, springflow, and groundwater sites in the West Nueces, Nueces, Frio, and Sabinal River watersheds during two surveys in August 2009 and March 2010 (app. 2). Water-quality samples were collected by following standard USGS methods (U.S. Geological Survey, variously dated). Depth-integrated streamflow samples were collected during base-flow conditions either by multiple verticals using a hand-held sampler when stream velocities were less than about 1.5 feet per second (ft/s) or by the flow-weighted, equal-width increment method (Edwards and Glysson, 1999) when stream velocities were greater than about $1.5 \mathrm{ft} / \mathrm{s}$. Depending on springflow rates, springflow samples were collected by using a hand-held sampler or a grab sample from the spring orifice (U.S. Geological Survey, variously dated). Groundwater samples were collected from a spigot on top of the well if a submersible pump was already installed in the well. If the well did not have an installed pump, the well was purged and the groundwater sample was collected by using a submersible piston pump (U.S. Geological Survey, variously dated).

Physical properties including water temperature, dissolved oxygen, specific conductance, $\mathrm{pH}$, turbidity, and alkalinity were measured in the field at the time of sample collection during both surveys in August 2009 and March 2010. Samples collected in August 2009 were analyzed for major ions and strontium isotopes (strontium-87/strontium-86 $\left.\left[{ }^{87} \mathrm{Sr} /{ }^{86} \mathrm{Sr}\right]\right)$. Samples collected in March 2010 were analyzed for major ions, trace elements, and environmental isotopes including hydrogen (hydrogen-2/hydrogen- $1\left[{ }^{2} \mathrm{H} /{ }^{1} \mathrm{H}\right]$ ), oxygen (oxygen-18/oxygen-16 $\left[{ }^{18} \mathrm{O} /{ }^{16} \mathrm{O}\right]$ ), and strontium $\left({ }^{87} \mathrm{Sr} /{ }^{86} \mathrm{Sr}\right.$ ).

Samples collected for major ion and trace element analyses were filtered in the field by using a 0.45 -micron filter, preserved with nitric acid to a $\mathrm{pH}$ of less than 2 , and shipped at 4 degrees Celsius to the USGS National Water Quality Laboratory (NWQL) in Denver, Colorado, for analysis. Major ions, silica, dissolved solids, and trace elements were analyzed by following the methods described in Fishman and Friedman (1989), Fishman (1993), American Public Health Association (1998), Garbarino (1999), and Garbarino and others (2006).

The ratio of the naturally occurring, stable isotopes of strontium $\left({ }^{87} \mathrm{Sr} r{ }^{86} \mathrm{Sr}\right)$ was measured in the August 2009 and March 2010 samples by the USGS National Research Program Laboratory in Menlo Park, California, by following the methods described in Bullen and others (1996) and is reported as a dimensionless ratio. The laboratory reported ${ }^{87} \mathrm{Sr} /{ }^{86} \mathrm{Sr}$ ratio for standard reference material (SRM) 987 from the National Institute of Standards and Technology as $0.71024(0.00002$ 2-sigma, 95 percent confidence level). Samples collected in March 2010 also were analyzed for oxygen and hydrogen isotopes $\left({ }^{18} \mathrm{O} /{ }^{16} \mathrm{O},{ }^{2} \mathrm{H} /{ }^{1} \mathrm{H}\right)$ by the USGS Stable Isotope Laboratory in Reston, Virginia, by following the methods described in Révész and Coplen (2008a, b) and are reported in per mil relative to Vienna Standard Mean Ocean Water (VSMOW).

The reporting of results for major ion and trace elements is described by Oden and others (2011, p. 9):

The analytical quantification procedure used by the NWQL for reporting results is based on the long-term method detection level (LT-MDL) and laboratory reporting level (LRL). The LT-MDL concentrations are defined as a censoring limit for most analytical methods at the NWQL, and its purpose is to limit the false positive rate to less than or equal to 1 percent. An LT-MDL is a modification of the USEPA 40 Code of Federal Regulations Part 136 definition of the method detection limit (MDL). The LRL is defined as twice the LT-MDL and is established to limit the occurrence of false negative detections to less than or equal to 1 percent (Childress and others, 1999). A constituent concentration is considered estimated by the laboratory when results are greater than the LT-MDL and less than the LRL; that is, a detection 
is considered likely, but quantification is considered questionable. The remark code of " $\mathrm{E}$ " (estimated) is assigned by the laboratory for these results.

\section{Quality Assurance of Water-Quality Samples}

Quality-control samples were collected at streamflow and springflow sites during August 2009 and March 2010 by following USGS procedures for the collection and processing of water-quality samples (U.S. Geological Survey, variously dated). The purpose of collecting quality-control samples is to evaluate results from the water-quality samples with respect to quality, precision, accuracy, and completeness. The types of quality-control samples collected for this study included an equipment blank, field blanks, and sequential replicates (app. 3).

An equipment blank sample was collected on August 10,2009 , prior to the start of sampling. The equipment blank was identified with U.S. Geological Survey station number 294424099454101 (app. 3), though an environmental sample was not collected from this site. Analytical results from the equipment blank on August 10, 2009, indicate that only manganese was detected, with an estimated concentration of 0.2 milligrams liter $(\mathrm{mg} / \mathrm{L})$. All other analyzed constituent concentrations were less than the LRL.

Two field blank samples were collected during the sampling period (August 14, 2009, and March 22, 2010). For the August 14, 2009 sample, the estimated concentration of $0.07 \mathrm{mg} / \mathrm{L}$ for chloride was approximately two orders of magnitude less than the chloride concentrations measured in the August 14, 2009, environmental samples (apps. 2 and 3). All other analyzed constituent concentrations were less than the LRL in the August 14, 2009, sample. Calcium, chloride, silica, cobalt, manganese, nickel, zinc, and antimony were detected in the field blank sample collected on March 22, 2010 (app. 3). The concentrations of calcium, chloride, silica, nickel, and antimony were less than concentrations measured in the environmental samples, indicating that possible contamination likely did not affect the concentrations of these constituents in the environmental samples. Cobalt, manganese, and zinc were detected in the field blank sample at concentrations that exceeded the concentrations measured in some of the environmental samples (app. 3). For these constituents, the laboratory reported concentrations for the environmental samples are included in appendix 2 for completeness; however, the concentrations might be affected by possible contamination.

Sequential replicate samples were collected from site 64 in August 2009 and site 62 in March 2010 (app. 3).

Sequential replicate samples were compared to the associated environmental samples by calculating the RPD for each pair of detected constituents, where the variable of interest was concentration (eq. 1; app. 3). The RPD was not computed for a constituent pair if either of the values was reported as less than the LRL or if either of the values was reported as estimated values. RPDs of 10 percent or less were used to indicate good agreement between analytical results if the concentrations were sufficiently large compared to the LRL. RPDs were calculated for 19 constituents by using environmental and sequential replicate samples collected from site 64 in August 2009 (app. 3). The calculated RPDs for August 2009 were 10 percent or less for all constituents, indicating that there was good agreement and reproducibility between the environmental sample values and the sequential-replicate values. RPDs were calculated for 30 constituents by using environmental and sequential replicate samples collected from site 62 in March 2010. The calculated RPDs for March 2010 that exceeded 10 percent were fluoride (17 percent), cobalt (21 percent), nickel (11 percent), and selenium (26 percent) (app. 3).

\section{Streamflow Gain and Loss}

During the 2008, 2009, and 2010 surveys, reaches of gaining, losing, or no change in streamflow were identified on the West Nueces, Nueces, Frio, Dry Frio, and Sabinal Rivers (figs. 5-7, table 1). On the basis of the three surveys, reaches of generally consistent gaining or losing streamflow were identified in the Nueces, Frio, and Sabinal River watersheds; the streamflow measurement data collected in the West Nueces and Dry Frio watersheds were insufficient to identify consistently gaining or losing reaches. Identifying reaches with consistent gains and losses during the three surveys is related in part to the measurement site locations, and differences in some site locations among surveys were considered when identifying specific reaches where streamflow gains or losses occurred. Changes in hydrologic conditions also might contribute to differences in observed streamflow gains and loss patterns. For example, several alternating reaches of gains and losses were observed during the 2008 and 2009 surveys (low-flow and very low flow conditions, respectively). During the 2010 survey (mediumflow conditions), some reaches exhibited similar patterns compared to those observed in 2008 and 2009; however, there were fewer losing reaches and more gaining reaches in 2010 compared to the previous two surveys (possibly because of increase in springflows during medium-flow conditions as compared to low-flow and very low flow conditions). Because of differences in streamflow during each survey and because different measurement sites were used in each survey, general patterns of streamflow gains and losses are emphasized rather than absolute magnitudes of streamflow gains and losses.

The headwaters of the Nueces River include Hackberry Creek and East Prong Nueces River. From site 13, downstream from the confluence of Hackberry Creek and East Prong Nueces River, to site 14, Nueces River streamflow decreased during the 2008 survey (fig. 5), though the difference was smaller than the sum of the potential measurement errors and thus is not a verifiable loss. The reach from site 14 to site 19 


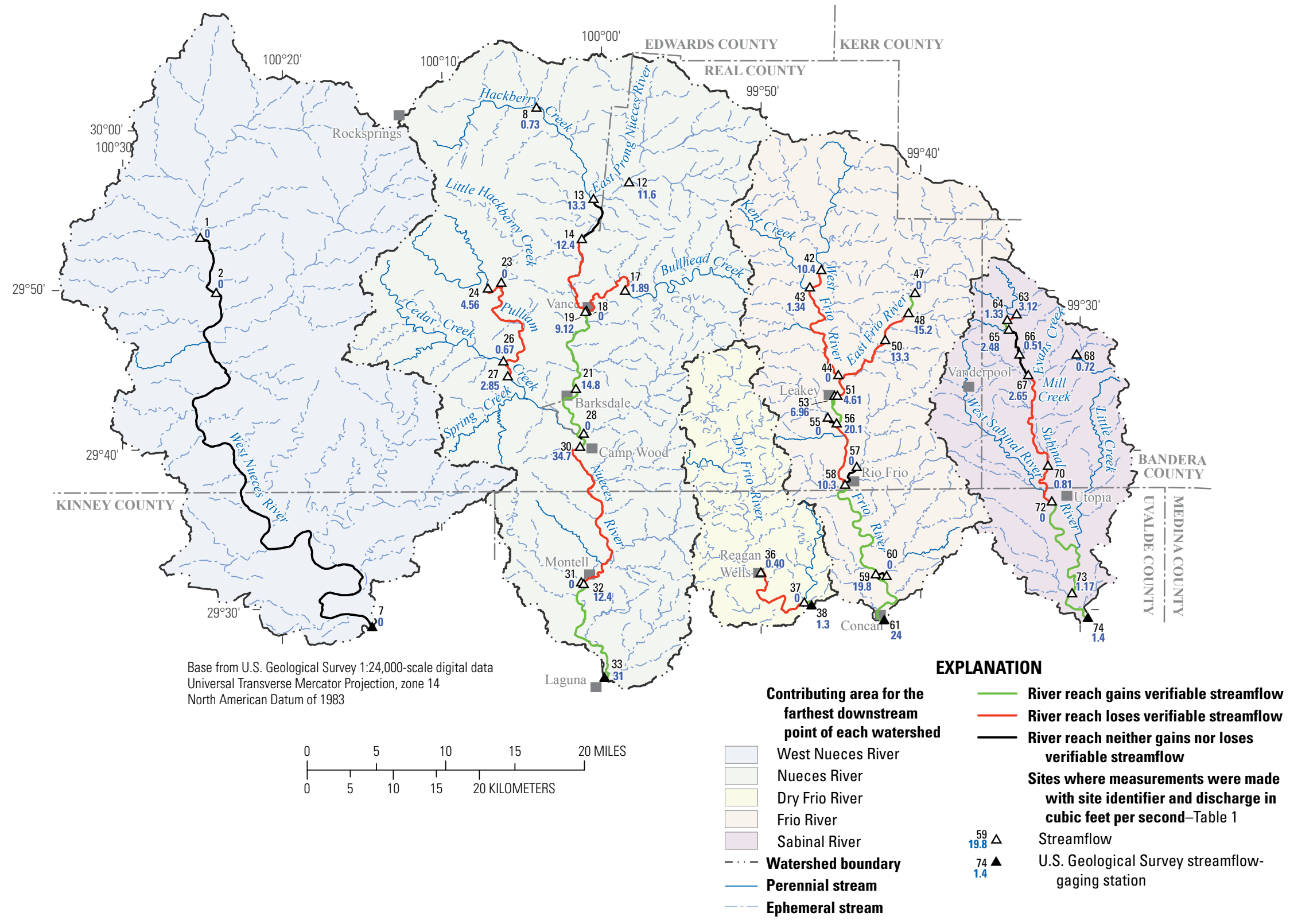

Figure 5. Streamflow measurements in the upper Nueces River Basin, south-central Texas, during July 2008. 


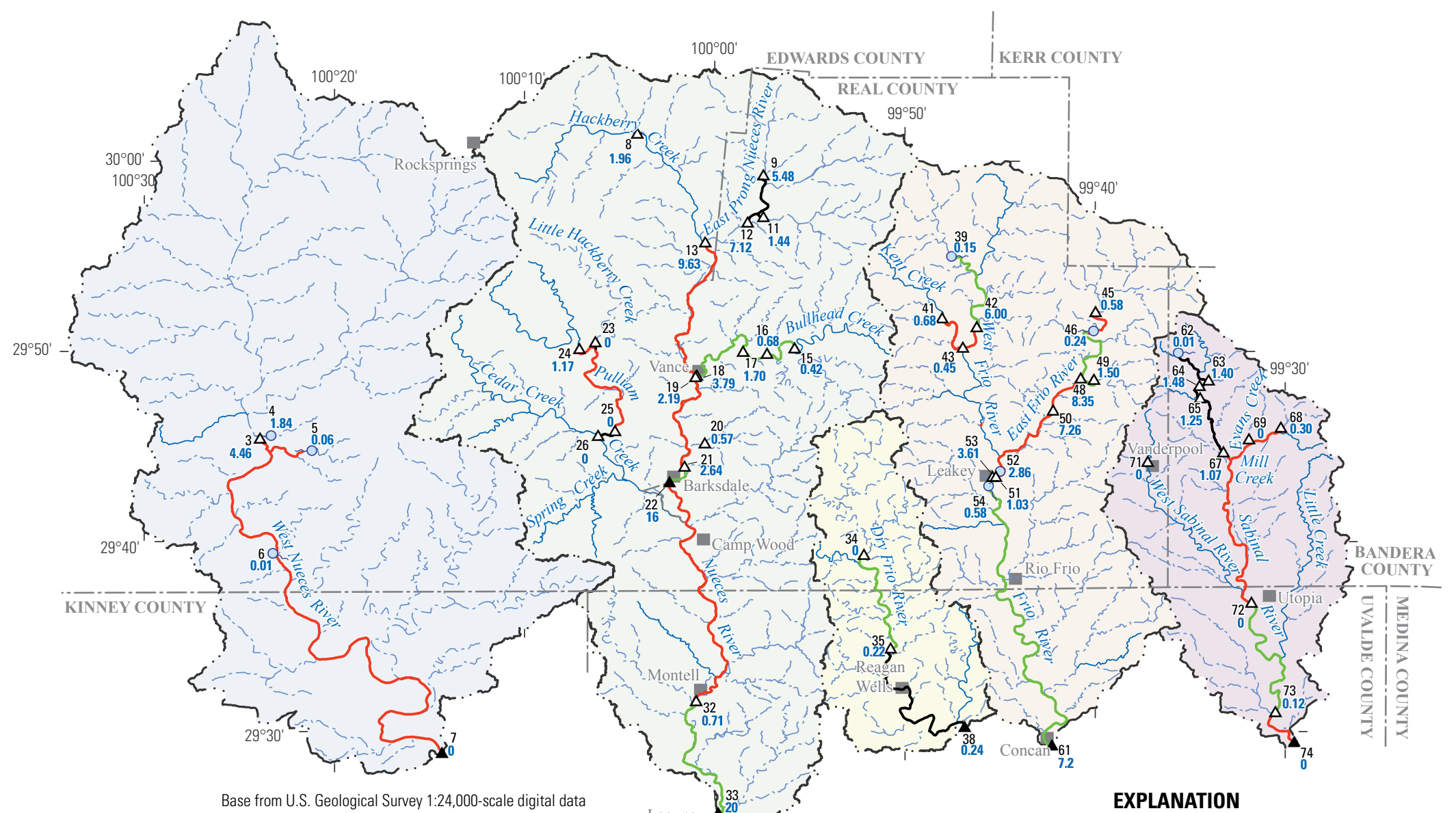

Base from U.S. Geological Survey 1:24,000-scale digital data Universal Transverse Mercator Projection, zone 14

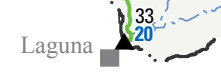

EXPLANATION

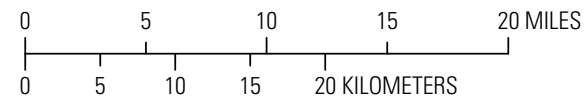

Contributing area for the most downstream point of each watershed

West Nueces River

Nueces River

Dry Frio River

Frio River

Sabinal River

-.. - Watershed boundary

Perennial stream

Ephemeral stream
River reach gains verifiable streamflow River reach loses verifiable streamflow River reach neither gains nor loses verifiable streamflow

Sites where measurements were made or samples were collected with site identifier and discharge in cubic feet per second-Table 1

${ }_{7.26}^{50} \Delta \quad$ Streamflow

${ }_{72}^{61}$ U.S. Geological Survey streamflowgaging station

${ }_{1.84}^{4}$ on Springflow-Tail points in direction of flow 


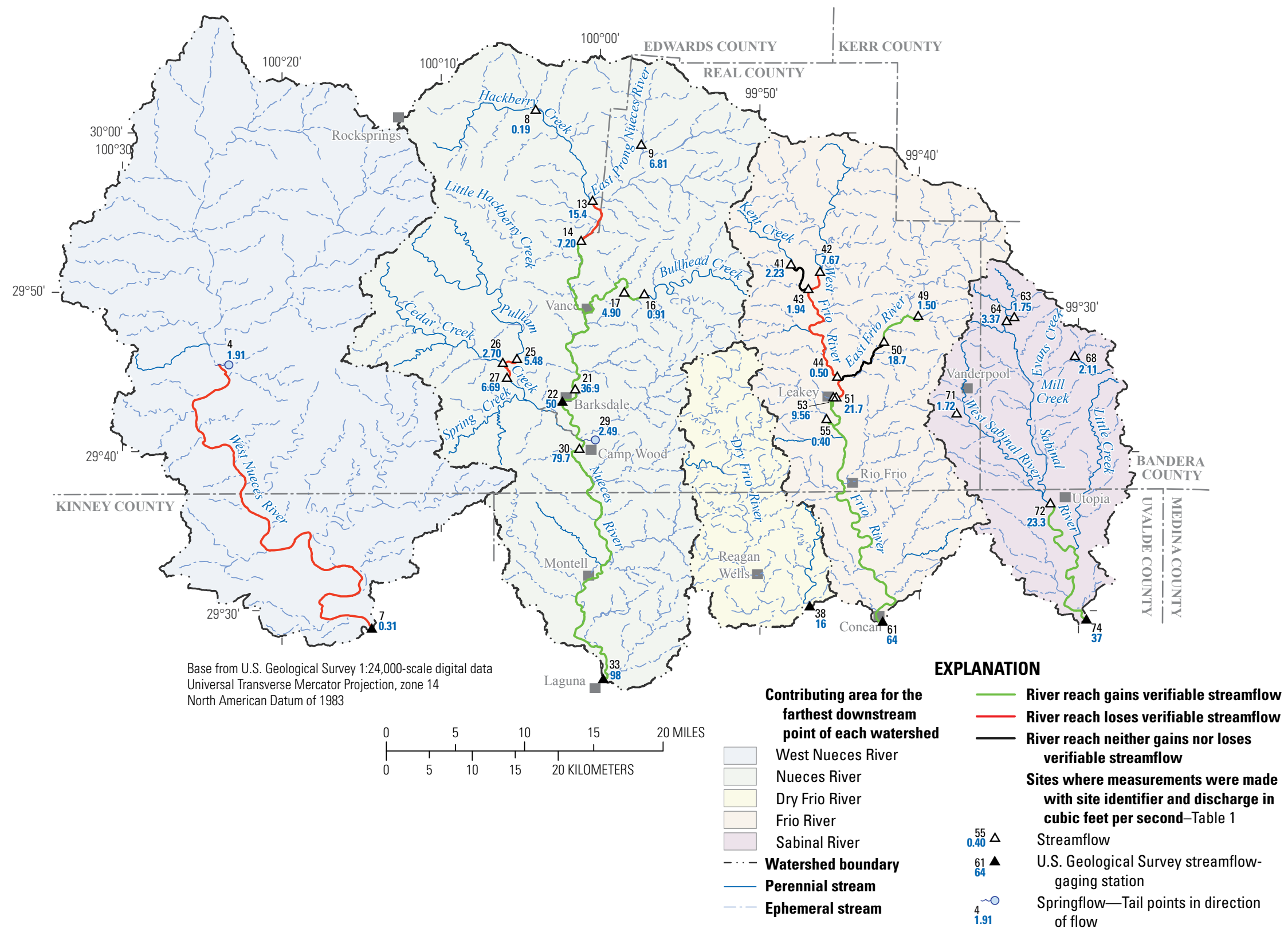

Figure 7. Streamflow and springflow measurements in the upper Nueces River Basin, south-central Texas, during March 2010. 
during the 2008 survey did exhibit a verifiable loss. During the 2009 survey (fig. 6), a verifiable loss was identified between sites 13 and 19, though measurements were not made at site 14. During the 2010 survey (fig. 7), measurements were not made at sites 18 or 19 , so it is not possible to confirm this pattern. A verifiable loss was identified, however, between sites 13 and 14 during the 2010 survey (similar to the 2008 survey), so it is possible that a consistent loss in streamflow occurs from site 13 to 19 .

From site 19 to site 21, the Nueces River was gaining streamflow during the 2008 survey and losing during the 2009 survey. Differences in measurement locations between surveys prevent direct comparisons with the 2010 survey, but measurements made at site 14 (upstream from site 19) to site 21 indicate that this was a gaining reach during the 2010 survey. Additionally, the 2009 and 2010 surveys indicate a substantial gain in streamflow occurring between sites 21 and 22.

From site 22 to site 30 , streamflow might be affected by potential inflows from Pulliam Creek. Pulliam Creek is identified as a perennial stream in the National Hydrography Dataset (U.S. Geological Survey, 2012b), and some measurements were made in the upper reaches but not at the confluence with the Nueces River during the surveys.

From site 30 downstream to site 32, the Nueces River was losing streamflow during the 2008 survey. Of the calculated streamflow losses, the largest streamflow loss between any two sites occurred in this reach during the 2008 survey $\left(22.3 \mathrm{ft}^{3} / \mathrm{s}\right)$, when $34.7 \mathrm{ft}^{3} / \mathrm{s}$ was measured at site 30 and $12.4 \mathrm{ft}^{3} / \mathrm{s}$ was measured at site 32 . Site 30 was not measured during the 2009 survey, so it is not possible to assess the consistency of loss under different hydrologic conditions; however, the reach from site 22 to 32 did exhibit a loss during this survey. The farthest downstream reach of the Nueces River in the study area, from site 32 to site 33, exhibited gains in streamflow during the 2008 and 2009 surveys. During the 2010 survey, a gain in streamflow was identified between sites 30 and 33, though it is not known where in that reach the gains occurred because a measurement was not made at site 32 .

Similar to the Nueces River, the Frio River exhibited patterns of reaches alternating between gaining and losing streamflow. The West Frio River and East Frio River originate from springs in the headwaters and then exhibit gaining and losing reaches downstream. Notably, the West Frio River was a losing stream from site 42 to site 44 during the 2008 and 2010 surveys. During the 2009 survey (very low flow conditions), site 44 was not measured. Because no flow was observed at site 44 during the 2008 survey (low-flow conditions), and because the reach from site 42 to site 44 during the 2010 survey (medium-flow conditions) exhibited a loss, it is likely that a loss was also present in 2009 (very low flow conditions). The East Frio River was a losing stream from site 50 to site 51 during the 2008 and 2009 surveys and neither gaining nor losing during the 2010 survey. In the reaches near Leakey (approximately site 51 to site 56), springs contribute to the Frio River streamflow (for example, Leakey Springs). During each of the surveys, the Frio River was a gaining stream from site 51 to the farthest downstream measurement site in the Frio River watershed study area (site 61). Along this reach, between the upstream and downstream sites used to characterize a gain or loss in streamflow in 2009 and 2010, there were additional sites where streamflow was measured during the 2008 survey, one of which was identified as a losing reach (site 56 to site 58 ). Because these additional sites were not measured during the 2009 and 2010 surveys, it is not possible to confirm these additional gaining or losing reaches identified in 2008.

Compared to the streamflow measured at the USGS streamflow-gaging stations on the Nueces and Frio Rivers (sites 33 and 61, respectively), measured streamflow was lower at the USGS streamflow-gaging station on the Sabinal River (site 74) during the three surveys. Upstream from site 72 on the Sabinal River, the reaches were generally losing or were neither gaining nor losing streamflow during the 2008 and 2009 surveys. No flow was observed at site 72 , and the Sabinal River was gaining in the reach between site 72 and site 73 during the 2008 and 2009 surveys. The increase in flow between these sites might be caused by inflows from Little Creek, by groundwater inflows, or by both. Little Creek is identified as a perennial stream in the National Hydrography Dataset (U.S. Geological Survey, 2012b), but it is not known if Little Creek was flowing at the confluence with the Sabinal River during the surveys. During the 2010 survey, the reach between site 72 and site 74 was gaining, though again, not all of the same sites were measured as in previous surveys, and potential contributions from Little Creek are not known.

To help understand streamflow gains and losses, groundwater levels (which might be indicative of groundwater contributions to streamflow) near the headwaters of the Nueces River (site 76) and streamflow in the Nueces River watershed (site 22) were examined for possible relations. A continuous groundwater-level monitor was installed at site 76, north of the northernmost measured streamflow and spring locations, in July 2009. Although a hydrologic connection between site 76 (a $398 \mathrm{ft}$ deep well) and the surface water has not been identified, some springs in the study area likely originate from the Edwards-Trinity (Plateau) aquifer (Long, 1962, Broad, 2011) - the same aquifer in which site 76 is completed. Hence, groundwater levels measured at site 76 might provide information pertaining to groundwater input to the springs in the area. In February 2009, a USGS continuous streamflow-gaging station was installed on the Nueces River near Barksdale, Tex. (site 22; fig. 1, table 1). This streamflow-gaging station is approximately halfway between the headwaters of the Nueces River and the downstream extent of the Nueces River watershed. A coefficient of determination $\left(\mathrm{R}^{2}\right)$ of 0.15 was calculated between the daily mean streamflow measured at site 22 and the daily mean groundwater levels at site 76 for the period of record. Though it is statistically significant (p-value was less than 0.01 ), the small $\mathrm{R}^{2}$ value indicates a relatively weak relation, and other processes might be contributing to the 
streamflow and groundwater measurements (the p-value is the probability of obtaining the computed $\mathrm{R}^{2}$ value if the null hypothesis of no relation between the streamflow at site 22 and groundwater levels measured at site 76 were true [Helsel and Hirsch, 2002]). It is important to note that there likely are several springs and tributaries contributing to the streamflow measured at site 22 . Additionally, the $\mathrm{R}^{2}$ does not take into account other factors, such as different lag times between surface water and groundwater in response to rainfall events and recession curves. Graphical comparisons of streamflow measured at site 22 and groundwater levels measured at site 76 indicate that, at times, streamflow at site 22 and groundwater levels at site 76 respond similarly to rainfall events measured at Rocksprings (site 78, National Climate Data Center, 2011), but more gradual responses and longer recession curves were observed for groundwater levels compared to streamflow (fig. 8). Although the results indicate a possible relation between groundwater levels and streamflow, additional data representing a larger range of hydrologic conditions are needed to confirm this relation.

\section{Water-Quality Data}

Water-quality samples were collected during the August 2009 and March 2010 surveys at selected sites in each watershed, except from the Dry Frio watershed (table 1, app. 2). The water-quality data were examined for possible relations among sample type (streamflow, springflow, or groundwater), between synoptic measurement surveys (to see if temporal differences were observed), and among watersheds in which the samples were collected.

The median specific conductance measured in the laboratory for all samples collected in the study area during 2008-10 was 410 microsiemens per centimeter $(\mu \mathrm{S} / \mathrm{cm})$, and the specific conductance ranged from 311 to $509 \mu \mathrm{S} / \mathrm{cm}$ for all but one sample (the exception was the specific conductance of $1,180 \mu \mathrm{S} / \mathrm{cm}$ measured in the groundwater sample collected from site 77). These results are consistent with previous USGS water-quality studies in south-central Texas (for example, Mahler, 2008; Musgrove and others, 2010).

Trilinear (Piper) diagrams were constructed for the waterquality samples collected during the 2009 and 2010 surveys (figs. 9 and 10) (Piper, 1944). These diagrams indicate that the streamflow, springflow, and groundwater have similar major ion chemical characteristics and generally can be categorized as a calcium-carbonate water type. The trilinear diagrams also indicate that the major ion chemistry was similar during the 2009 and 2010 surveys. The sampling events occurred during relatively stable hydrologic conditions (for example, no rain for 7 days prior to sampling) to reduce the potential influence of storm runoff on the water chemistry. During periods of base-flow conditions, the chemical characteristics of the streamflow, springflow, and groundwater samples are likely similar because streamflow in the study area is composed primarily from springs throughout the upper
Nueces River Basin, some of which likely originate from the Edwards-Trinity (Plateau) aquifer (Long, 1962; Broad, 2011). Similarities in water quality might indicate possible connectivity between groundwater and springs that originate in the study area, though the wells at sites 75 and 76 are deep (502 and $398 \mathrm{ft}$ deep, respectively) and known mechanisms of hydrologic connection between the groundwater at sites 75 and 76 with the springs in the study area are unknown.

Site 75 is just outside the study area (approximately 3 miles to the west). Site 75 is completed in the EdwardsTrinity (Plateau) aquifer, which might be contributing to springflow in the study area (Long, 1962; Broad, 2011). The trilinear diagrams (and subsequent isotopic ratio figs. 11-13) indicate that the water chemistry in the groundwater sample collected from site 75 is similar to the water chemistry from nearby springflow samples collected in the West Nueces River watershed. Hence, site 75 is included with samples from the West Nueces River watershed in subsequent discussions for this report.

The trilinear diagram depicting the chemical concentrations for the groundwater sample collected from site 77 in August 2009 indicates different water-quality characteristics compared to those for the remaining waterquality samples (fig. 9). This difference might be in part because the well is completed in the Trinity aquifer, which is a different aquifer from the Edwards-Trinity (Plateau) aquifer in which the other wells were completed. Hence, water-quality data collected from site 77 are not included in the subsequent analyses but are included in appendix 2 and figure 9 for completeness.

To further evaluate temporal (between surveys) and spatial (among watersheds) comparisons, the water chemistry can be examined in regard to elemental and isotopic ratios. Relations among ratios of major ions, trace elements, or isotopes have be used in previous studies to evaluate groundwater geochemical processes of water-rock interactions such as geochemical evolution (Trudgill, 1995; Oetting and others, 1996; Clark and Fritz, 1997; Sharp and Banner, 1997; Musgrove and Banner, 2004; Musgrove and others, 2010). Water-rock interactions are the geochemical processes between the fluid and host rock matrix. For example, Musgrove and others (2010) assessed geochemical evolution in carbonate groundwater systems and found that increased residence times or longer flow paths were often associated with increased magnesium to calcium $(\mathrm{Mg} / \mathrm{Ca})$ molar ratio and strontium to calcium $(\mathrm{Sr} / \mathrm{Ca})$ molar ratio, and decreased strontium isotopic ratios.

The relations of $\mathrm{Mg} / \mathrm{Ca}$ molar ratios to Ca concentrations from all available samples (streamflow, springflow, and groundwater samples) collected from four of the watersheds in the study area during two surveys (August 2009 and March 2010) are depicted in figure 11 . The $\mathrm{Mg} / \mathrm{Ca}$ molar ratio to calcium concentrations from the samples generally plotted in clusters according to the watershed from which the samples were collected. In general, lower $\mathrm{Mg} / \mathrm{Ca}$ molar ratios were measured in samples from the West Nueces River watershed 

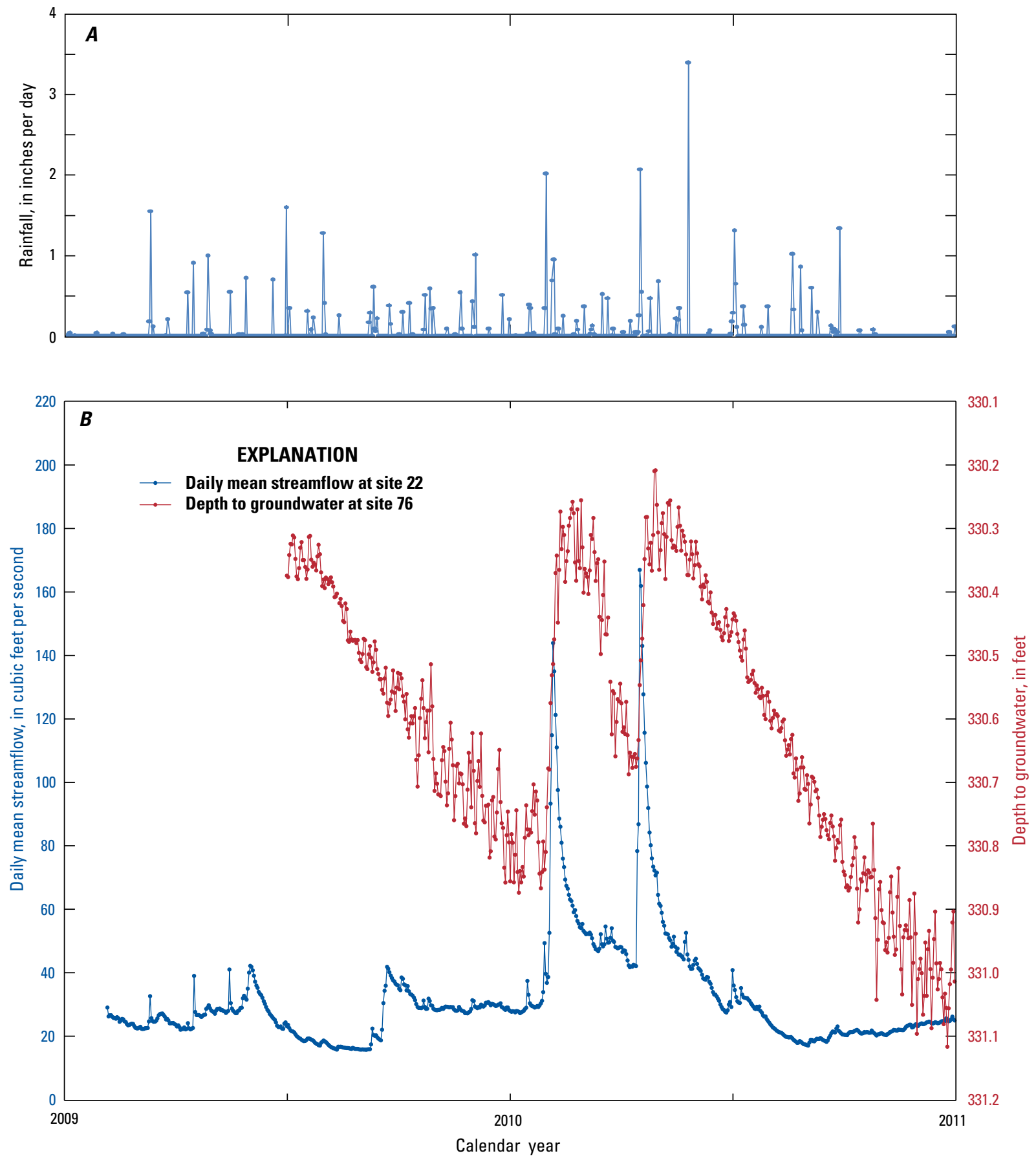

Figure 8. Comparisons of measured rainfall, streamflow, and groundwater levels in the Nueces River watershed of the upper Nueces River Basin study area, south-central Texas, 2009-10. A, Rainfall at site 78 (National Weather Service station 417706, Rocksprings 1S), in Rocksprings, January 2009-December 2010. B, Daily mean streamflow at site 22 (U.S. Geological Survey streamflow-gaging station 0818999010) and groundwater levels, reported as depth to water, at site 76 (U.S. Geological Survey station 300259100030801), July 2009December 2010. 


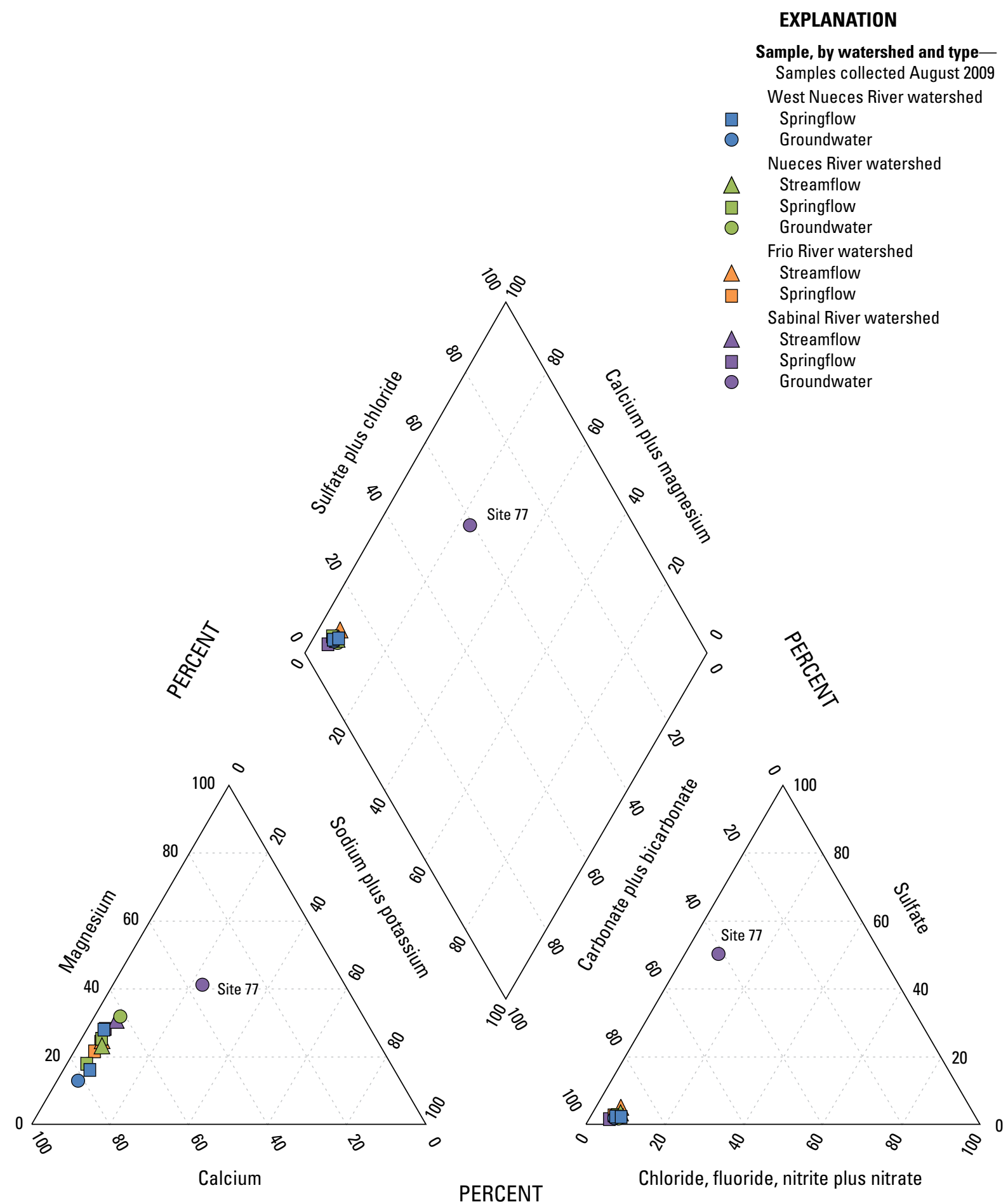

Note: Site 77 represents U.S. Geological Survey station 294815099343801

Figure 9. Trilinear diagrams of chemical composition for streamflow, springflow, and groundwater samples collected in the upper Nueces River Basin, south-central Texas, August 2009. 


\section{EXPLANATION}

Sample, by watershed and typeSamples collected March 2010

West Nueces River watershed

Springflow

Groundwater

Nueces River watershed

Streamflow

Springflow

Frio River watershed

Streamflow

Springflow

Sabinal River watershed

Streamflow

Springflow

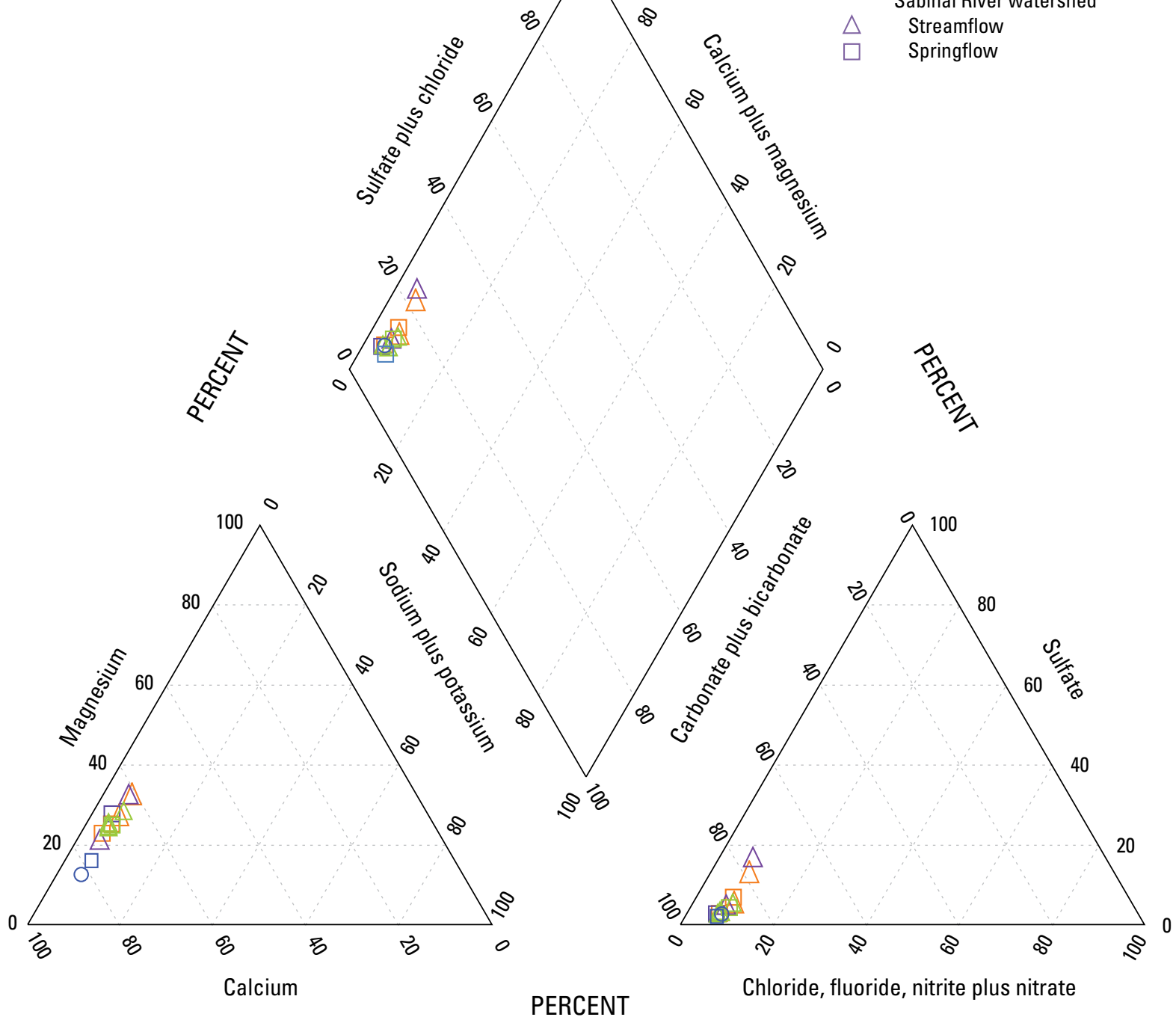

Figure 10. Trilinear diagrams of chemical composition for streamflow, springflow, and groundwater samples collected in the upper Nueces River Basin, south-central Texas, March 2010. 


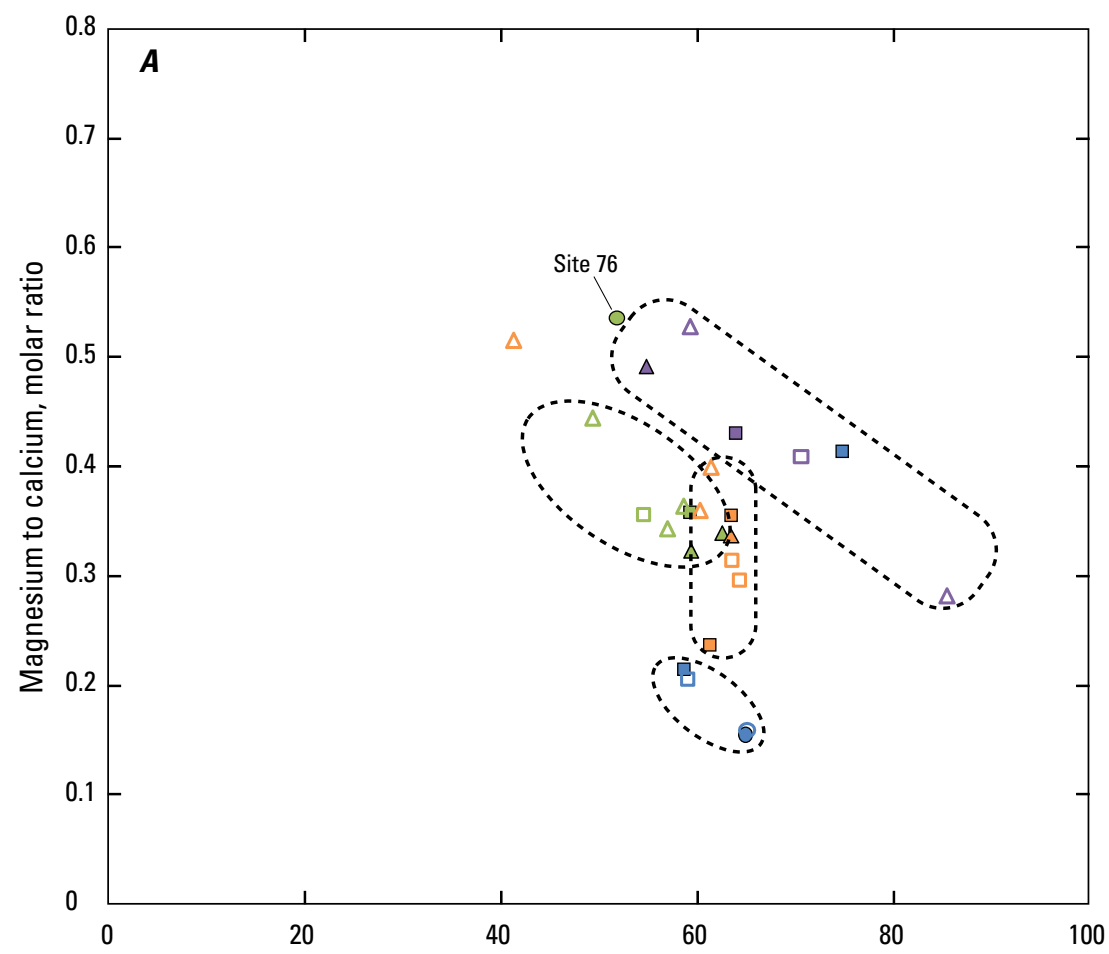

\section{EXPLANATION}

-...- Cluster

Sample, by watershed and typeFilled symbols represent samples collected August 2009.

Open symbols represent samples collected March 2010

West Nueces River watershed Springflow

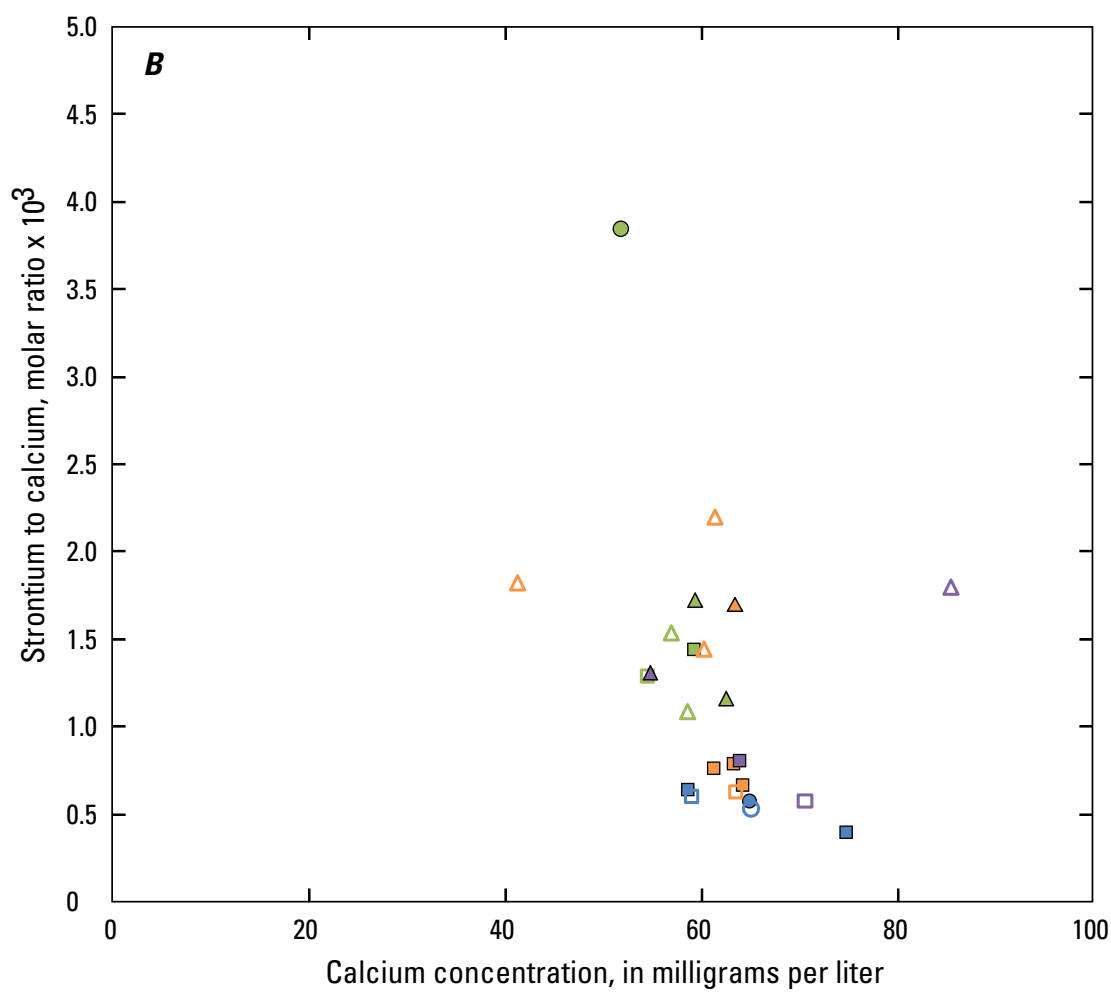

- Groundwater

Nueces River watershed

Streamflow

Springflow

Groundwater

Frio River watershed

$\Delta \quad$ Streamflow

$\square \quad$ Springflow

Sabinal River watershed

$\Delta \quad$ Streamflow

$\square \quad$ Springflow

Note: Site 76 represents U.S. Geological Survey station 300259100030801

Figure 11. Relations of molar ratios to calcium concentrations for streamflow, springflow, and groundwater samples collected in the upper Nueces River Basin, south-central Texas, August 2009 and March 2010. $A$, Relation between magnesium to calcium molar ratio and calcium concentration. $B$, Relation between strontium to calcium molar ratio and calcium concentration. 


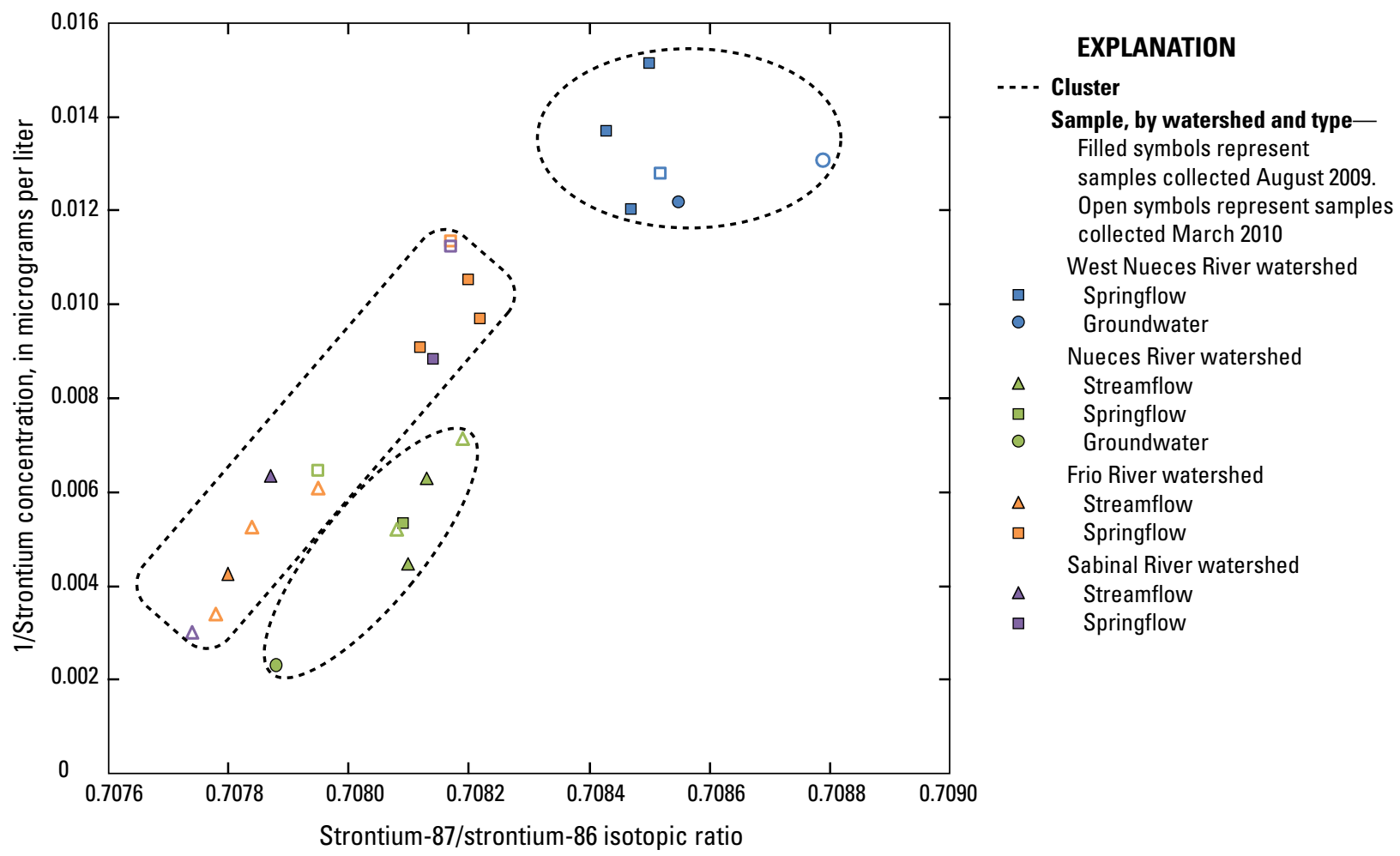

Figure 12. Relation of inverse strontium concentration to strontium-87/strontium-86 isotopic ratio for streamflow, springflow, and groundwater samples collected in the upper Nueces River Basin, south-central Texas, August 2009 and March 2010.

compared to those from the other watersheds. This indicates the water samples obtained from the West Nueces River watershed might be less geochemically evolved. Compared to the other watersheds, samples from the Sabinal River watershed generally exhibited higher $\mathrm{Mg} / \mathrm{Ca}$ molar ratios. $\mathrm{The} \mathrm{Mg} / \mathrm{Ca}$ molar ratio to calcium concentration relation for samples did not always plot within the corresponding watershed from which the sample was collected. These differences might be in part related to the media type of the sample. For example, the $\mathrm{Mg} / \mathrm{Ca}$ molar ratio of the groundwater sample collected in the Nueces River watershed (site 76) plotted close to the $\mathrm{Mg} / \mathrm{Ca}$ molar ratios measured in samples collected from Sabinal River watershed (fig. 11A). Given the small sample size, it is difficult to identify the source of discrepancies in $\mathrm{Mg} / \mathrm{Ca}$ molar ratio to calcium concentrations for samples collected from a given watershed. Additionally, while plots of molar ratios sometimes exhibited general clusters by watershed, a clear spatial pattern was not always observed. For example, the $\mathrm{Sr} / \mathrm{Ca}$ molar ratio to calcium concentrations from samples did not cluster by watershed (fig. $11 B$ ). The different relations among the water-quality data indicate that the surface water in the different watersheds is likely influenced by a combination of differences in water-rock interactions and geochemical evolution (Trudgill, 1995; Oetting and others, 1996; Clark and Fritz, 1997; Musgrove and Banner, 2004; Musgrove and others, 2010).
Strontium isotopes $\left({ }^{87} \mathrm{Sr} /{ }^{86} \mathrm{Sr}\right)$ can also be an indicator of water-rock interactions and geochemical evolution (Trudgill, 1995; Clark and Fritz, 1997; Banner, 2004; Musgrove and others, 2010). Figure 12 depicts the relation of the inverse strontium concentration (1/Strontium) to the ${ }^{87} \mathrm{Sr} /{ }^{86} \mathrm{Sr}$ ratio for samples collected from different watersheds in the study area during the 2009 and 2010 surveys. Samples collected from the West Nueces River and Nueces River watersheds generally clustered separately. Samples collected from the Frio and Sabinal River watersheds plotted in a single cluster. Although the reasons for these differences are not fully understood, they might be caused in part by groundwater water-rock interactions which can vary with local lithology (Trudgill, 1995; Clark and Fritz, 1997; Musgrove and Banner, 2004; Musgrove and others, 2010). The relations between $\mathrm{Mg} / \mathrm{Ca}$ molar ratio and ${ }^{87} \mathrm{Sr} /{ }^{86} \mathrm{Sr}$ isotopic ratio (fig. $13 A$ ) and $\mathrm{Sr} / \mathrm{Ca}$ molar ratio and ${ }^{87} \mathrm{Sr} /{ }^{86} \mathrm{Sr}$ isotopic ratio (fig. $13 B$ ) for samples collected from different watersheds in the study area during the August 2009 and March 2010 surveys are depicted in figure 13. Compared to samples from the other watersheds, higher ${ }^{87} \mathrm{Sr} /{ }^{86} \mathrm{Sr}$ isotopic ratios were measured in the samples collected in the West Nueces River watershed. The relation between the $\mathrm{Mg} / \mathrm{Ca}$ molar ratio and ${ }^{87} \mathrm{Sr} /{ }^{86} \mathrm{Sr}$ isotopic ratio indicates that the samples collected from the West Nueces River watershed were chemically different from the others; however, samples from the other watersheds do not form distinct clusters (fig. 13A). Similar to the clusters of analytical 

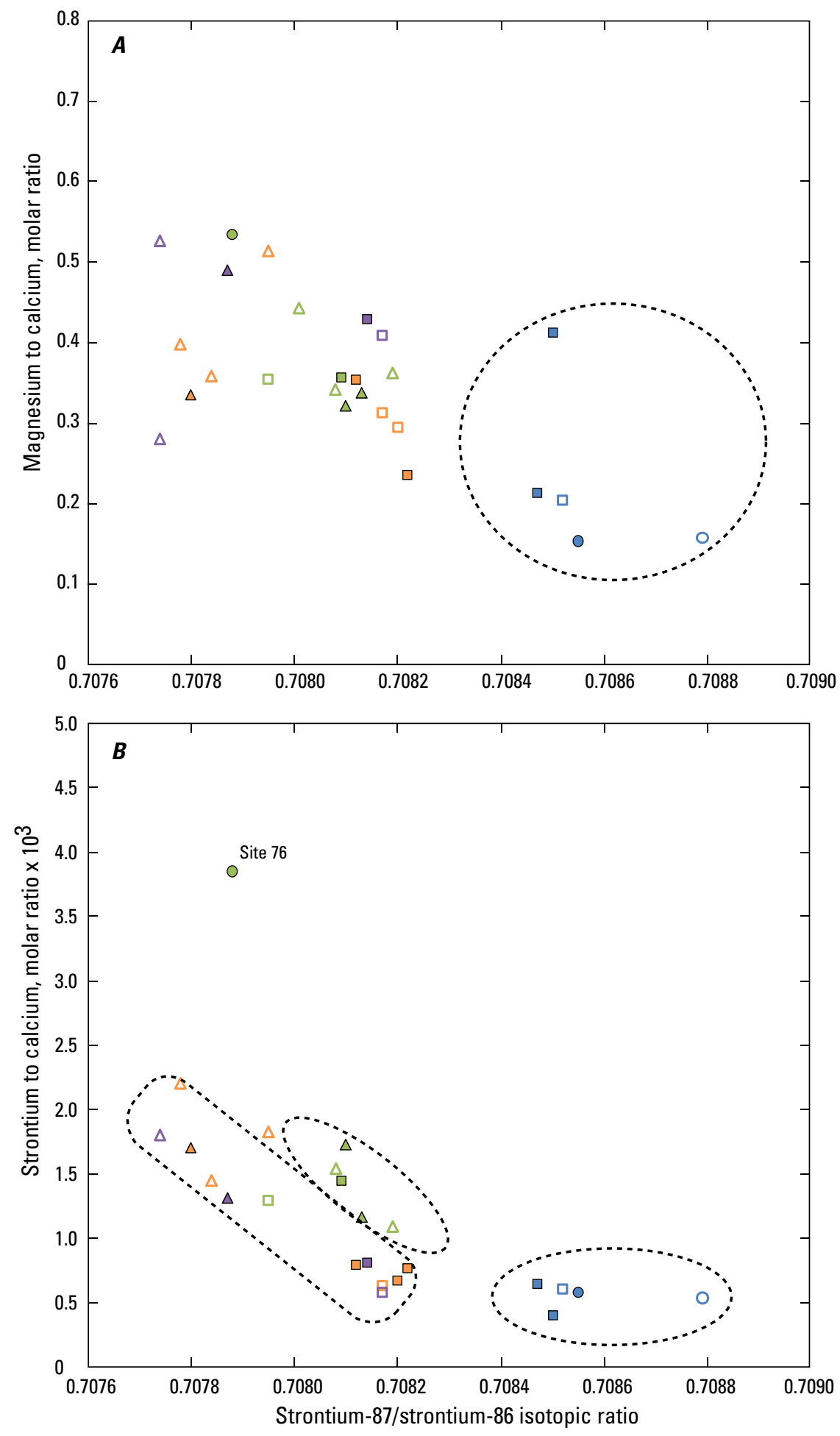

\section{EXPLANATION}

.... Cluster

Sample, by watershed and type-

Filled symbols represent

samples collected August 2009.

Open symbols represent samples collected March 2010

West Nueces River watershed

Springflow

Groundwater

Nueces River watershed

Streamflow

Springflow

Groundwater

Frio River watershed

$\triangle \quad$ Streamflow

$\square \quad$ Springflow

Sabinal River watershed

$\triangle \quad$ Streamflow

- Springflow

Note: Site 76 represents U.S. Geological Survey station 300259100030801

Figure 13. Relations between molar ratios and strontium-87/strontium-86 isotopic ratios for streamflow, springflow, and groundwater samples collected in the upper Nueces River Basin, south-central Texas, August 2009 and March 2010. A, Magnesium to calcium molar ratio to strontium-87/strontium- 86 isotopic ratio. $B$, Strontium to calcium molar ratio and strontium-87/strontium- 86 isotopic ratio. 
results depicted in figures 11 and 12, the Nueces, Frio, and Sabinal River watershed samples generally plotted in clusters in fig. $13 B$. The groundwater sample collected in August 2009 from the Nueces River watershed (site 76) plots notably outside of the cluster for this watershed-likely because the groundwater was more geochemically evolved compared to the streamflow and springflow samples from the Nueces River watershed.

The different relations among the water-quality data (as shown in figs. 11-13) indicate that the water quality in the different watersheds is likely influenced by differences in water-rock interactions which can vary with local lithology, and geochemical evolution which is linked with groundwater flow paths and residence time, or some combination thereof. Determining the source areas and other possible contributors on the basis of these data is not possible because of the small sample size of the water-quality dataset (both in number of samples and spatial distribution of samples).

\section{Summary}

The U.S. Geological Survey (USGS) - in cooperation with the U.S. Army Corps of Engineers, The Nature Conservancy, the Real Edwards Conservation and Reclamation District, and the Texas Parks and Wildlife Department - investigated streamflow gain and loss and water quality in the upper Nueces River Basin, south-central Texas. The study area (2,152 square miles) consists of the contributing areas (watersheds) of five streams (equipped with a total of six USGS streamflow-gaging stations) in the upper Nueces River Basin, north of the Edwards aquifer outcrop. These contributing areas are referred to as the West

Nueces, Nueces, Dry Frio, Frio, and Sabinal River watersheds. Streamflow in these rivers is sustained by groundwater contributions (for example, from springs) and by storm runoff from rainfall events. To date (2012), there are few data available that describe streamflow conditions of these major rivers within the upper Nueces River Basin.

This report describes the results of streamflow sampling during three reconnaissance-level synoptic measurement surveys (hereafter, surveys) in the upper Nueces River Basin in south-central Texas during July 2008, August 2009, and March 2010 and water-quality sampling during August 2009 and March 2010. Streamflow and springflow measurements were collected at as many as 74 sites during each of the three survey periods, although not all sites were measured during each survey. These survey periods were selected to represent different hydrologic conditions - low flow, very low flow, and medium flow. In July 2008, when streamflow at the farthest downstream station in each of the five contributing watersheds was generally less than the 25 th percentile of long-term streamflow measured at these stations, the streamflow conditions were referred to as "low flow"; "very low flow" refers to conditions that existed in August 2009 when streamflow of the five rivers generally was less than the 10th percentile of the respective long-term streamflows; and "medium flow" refers to conditions that existed in March 2010 when streamflow was generally around the 50th percentile of long-term streamflow.

During the 2008, 2009, and 2010 surveys, reaches of gaining, losing, or no verifiable change in streamflow were identified on the West Nueces, Nueces, Frio, Dry Frio, and Sabinal Rivers. Reaches of generally consistent gaining or losing streamflow were identified in the Nueces, Frio, and Sabinal River watersheds. Identifying reaches with consistent gains and losses during the three surveys is related in part to the measurement site locations, some of which were different between the surveys, as well as to changes in hydrologic conditions. For example, several alternating reaches of gains and losses were observed during the 2008 and 2009 surveys (low-flow and very low flow conditions, respectively). During the 2010 survey (medium-flow conditions), some reaches exhibited similar patterns; however, there were fewer losing reaches and more gaining reaches.

To help understand streamflow gains and losses, groundwater levels (which might be indicative of groundwater contributions to streamflow) near the headwaters of the Nueces River (site 76, U.S. Geological Survey station 300259100030801) and streamflow in the Nueces River watershed (site 22, U.S. Geological Survey station 0818999010) were examined for possible relations. Graphical comparisons of streamflow measured at site 22 and groundwater levels measured at site 76 indicate that, at times, streamflow at site 72 and groundwater levels at site 76 respond similarly to rainfall events measured at Rocksprings, Tex. (site 78, National Weather Service cooperative station number 417706), but more gradual responses and longer recession curves were observed for groundwater levels compared to streamflow.

The chemical data were examined for possible relations among sample types (streamflow, springflow, or groundwater), the timing of the synoptic measurement surveys (to see if temporal differences were observed), and the locations of the watersheds in which the samples were collected. Trilinear diagrams indicate that the streamflow, springflow, and groundwater have similar major ion chemical characteristics and can be categorized as a calcium-carbonate water type. The diagrams also indicate that the major ion chemistry was similar during the 2009 and 2010 surveys.

Relations between chemical concentrations and ratios can be used to evaluate groundwater geochemical processes of water-rock interaction. Graphical comparisons among ratios of major ions, trace elements, and isotopes (for example, magnesium/calcium ratios to strontium isotopic ratios) indicate that samples collected from each watershed generally clustered together. Determining the source areas and other possible contributors on the basis of these data is not possible because of the small sample size of the water-quality dataset (both in number of samples and spatial distribution of samples). The relations among the water-quality data indicate that the water quality in the different watersheds is likely influenced by differences in water-rock interactions, which 
can vary with local lithology and geochemical evolution, or some combination thereof.

\section{References}

American Public Health Association, 1998, Standard methods for the examination of water and wastewater (20th ed.): Washington, D.C., v. 2130, p. 2-8 to 2-11.

Ashworth, J.B., and Hopkins, Janie, 1995, Aquifers of Texas: Austin, Tex., Texas Water Development Board, Report 345.

Banner, J.L., 2004, Radiogenic isotopes-Systematics and applications to earth surface processes and chemical stratigraphy: Earth Science Reviews, v. 65, p. 141-194.

Barker, R.A., and Ardis, A.F., 1996, Hydrogeologic framework of the Edwards-Trinity aquifer system, west-central Texas, in Regional aquifer-system analysis-Edwards-Trinity aquifer system: U.S. Geological Survey Professional Paper 1421-B, 61 p., 8 pl.

Bayless, E.R., Bullen, T.D., and Fitzpatrick, J.A., 2004, Use of $87 \mathrm{Sr} / 86 \mathrm{Sr}$ and $\delta 11 \mathrm{~B}$ to identify slag-affected sediment in southern Lake Michigan: Environmental Science and Technology, v. 38, no. 5, p. 1330-1337.

Beck, D.A., and Wilson, J.W., 2006, Discharge and physicalproperty measurements from Virgin River Narrows, Arizona, to Lake Mead, Nevada, February 12, 2003: U.S. Geological Survey Scientific Investigations Report 20055286, accessed July 17, 2012, at http://pubs.water.usgs.gov/ sir2005-5286.

Braun, C.L., and Lambert, R.B., 2011, Streamflow, groundwater hydrology, and water quality in the upper Coleto Creek watershed in southeast Texas, 2009-10: U.S. Geological Survey Scientific Investigations Report 2011$5157,53 \mathrm{p}$.

Broad, Tyson, 2011, Water resources of the Bandera Canyonlands, Texas: Bandera Canyonlands Alliance, Vanderpool, Texas, $24 \mathrm{p}$.

Brown, N.K., 1965, Stratigraphy of Upper Cretaceous beds in the vicinity of D'Hanis, Medina County, Texas: Corpus Christi Geological Society Annual Field Trip Guidebook, p. 23-30.

Brown, T.E., 1963, Index to areal geologic maps in Texas, 1891-1961: The University of Texas at Austin, Bureau of Economic Geology, 20 p.

Bullen, T.D., Krabbenhoft, David, and Kendall, Carol, 1996, Kinetic and mineralogic controls on the evolution of groundwater chemistry and ${ }^{87} \mathrm{Sr} /{ }^{86} \mathrm{Sr}$ in a sandy silicate aquifer, northern Wisconsin: Geochimica et Cosmochimica Acta, v. 60, n. 10, p. 1807-1821.
Childress, C.J.O., Foreman, W.T., Conner, B.F., and Maloney, T.J., 1999, New reporting procedures based on long-term method detection levels and some considerations for interpretations of water-quality data provided by the U.S. Geological Survey National Water Quality Laboratory: U.S. Geological Survey Open-File Report 99-193, 19 p.

Clark, Ian, and Fritz, Peter, 1997, Environmental isotopes in hydrogeology: Boca Raton, Fla., CRC Press, 328 p.

Coplen, T.B., 1994, Reporting of stable hydrogen, carbon, and oxygen isotopic abundances: Pure and Applied Chemistry, v. 66, p. $273-276$.

Cunningham, W.L., and Schalk, C.W., comps., 2011, Groundwater technical procedures of the U.S. Geological Survey: U.S. Geological Survey Techniques and Methods 1-A1, $151 \mathrm{p}$.

Edwards, T.K., and Glysson, D.G., 1999, Field methods for measurement of fluvial sediment: U.S. Geological Survey Techniques of Water-Resources Investigations, book 3, chap. C2, 89 p.

Fahlquist, Lynne, and Ardis, A.F., 2004, Quality of water in the Trinity and Edwards aquifers, south-central Texas, 1996-98: U.S. Geological Survey Scientific Investigations Report 2004-5201, 17 p.

Fishman, M.J., ed., 1993, Methods of analysis by the U.S. Geological Survey National Water Quality LaboratoryDetermination of inorganic and organic constituents in water and fluvial sediments: U.S. Geological Survey OpenFile Report 93-125, 217 p.

Fishman, M.J., and Friedman, L.C., eds., 1989, Methods for determination of inorganic substances in water and fluvial sediments: U.S. Geological Survey Techniques of WaterResources Investigations, book 5, chap. A1, 545 p.

Freeman, L.A., Carpenter, M.C., Rosenberry, D.O., Rousseau, J.P., Unger, Randy, and McLean, J.S., 2004, Use of submersible pressure transducers in water-resources investigations: U.S. Geological Survey Techniques of Water-Resources Investigations, book 8, chap. A3, 52 p., accessed July 23, 2012, at http://pubs.usgs.gov/twri/twri8a3/ pdf/twri8-a3.pdf.

Garbarino, J.R., 1999, Methods of analysis by the U.S. Geological Survey National Water Quality LaboratoryDetermination of dissolved arsenic, boron, lithium, selenium, strontium, thallium, and vanadium using inductively coupled plasma-mass spectrometry: U.S. Geological Survey Open-File Report 99-093, 31 p.

Garbarino, J.R., Kanagy, L.K., and Cree, M.E., 2006, Determination of elements in natural-water, biota, sediment and soil samples using collision/reaction cell inductively coupled plasma-mass spectrometry: U.S. Geological Survey Techniques and Methods, book 5, sec. B, chap. 1, 88 p. 
Helsel, D.R., and Hirsch, R.M., 2002, Statistical methods in water resources: U.S. Geological Survey Techniques of Water-Resources Investigations, book 4, chap. A3, 510 p., accessed August 7, 2010, at http://pubs.usgs.gov/twri/ twri4a3/.

Kehew, Alan, 2001, Applied chemical hydrogeology: Upper Saddle River, N.J., Prentice-Hall, 368 p.

Larkin, T.J., and Bomar, G.W., 1983, Climatic atlas of Texas: Texas Department of Water Resources, Limited Printing Report LP-192, 151 p.

Long, A.T., 1962, Groundwater geology of Edwards County, Texas: Texas Water Commission, Bulletin 6208, 123 p.

Maclay, R.W., 1995, Geology and hydrology of the Edwards aquifer in the San Antonio area, Texas: U.S. Geological Survey Water-Resources Investigations Report 95-4186, $69 \mathrm{p}$.

Mahler, B.J., 2008, Statistical analysis of major ion and trace element geochemistry of water, 1986-2006, at seven wells transecting the freshwater/saline-water interface of the Edwards aquifer, San Antonio, Texas: U.S. Geological Survey Scientific Investigations Report 2008-5224, 46 p.

Musgrove, M., and Banner, J.L., 2004, Controls on the spatial and temporal variability of vadose dripwater geochemistry -Edwards aquifer, central Texas: Geochimica et Cosmochimica Acta, v. 68, p. 1007-1020.

Musgrove, M., Fahlquist, Lynne, Houston, N.A., Lindgren, R.J., and Ging, P.B., 2010, Geochemical evolution processes and water-quality observations based on results of the National Water-Quality Assessment Program in the San Antonio segment of the Edwards aquifer, 1996-2006: U.S. Geological Survey Scientific Investigations Report 2010-5129, 93 p.

National Climatic Data Center, 2011, Annual climatological summary for Rocksprings Station 1S: National Oceanic and Atmospheric Administration, accessed October 1, 2011, at http://cdo.ncdc.noaa.gov/dly/DLY.

Ockerman, D.J., 2002, Gain-loss study of lower San Pedro Creek and the San Antonio River, San Antonio, Texas, May-October 1999: U.S. Geological Survey Open-File Report 2002-023, 15 p.

Oden, J.H., Brown, D.W., and Oden, T.D., 2011, Groundwater quality of the Gulf Coast aquifer system, Houston, Texas, 2010: U.S. Geological Survey Data Series 598, 64 p.

Oetting, G.C., Banner, J.L., and Sharp, J.M., Jr., 1996, Geochemical evolution of saline groundwaters in the Edwards aquifer, central Texas - Regional stratigraphic, tectonic, and hydrodynamic controls: Journal of Hydrology, v. 181 , p. 251-283.
Piper, A.M., 1944, A graphic procedure in the geochemical interpretation of water analyses: Transactions, American Geophysical Union, v. 25, p. 914-923.

Rantz, S.E., and others, 1982a, Measurement and computation of streamflow-Volume 1 measurement of stage and discharge: U.S. Geological Survey Water-Supply Paper 2175, 284 p., accessed March 17, 2011, at http://pubs.usgs. gov/wsp/wsp2175/pdf/WSP2175_volla.pdf.

Rantz, S.E., and others, 1982b, Measurement and computation of streamflow-Volume 2 computation of discharge: U.S. Geological Survey Water-Supply Paper 2175, 631 p., accessed March 17, 2011, at http://pubs.usgs.gov/wsp/ wsp2175/pdf/WSP2175_vol2a.pdf.

Révész, Kinga, and Coplen, T.B., 2008a, Determination of the $\delta\left({ }^{18} 0 /{ }^{16} 0\right)$ of water-RSIL lab code 489 , chap. C2 of Révész, Kinga, and Coplen, T.B., eds., Methods of the Reston Stable Isotope Laboratory: U.S. Geological Survey Techniques and Methods, book 10, sec. C, chap. 2, 28 p.

Révész, Kinga, and Coplen, T.B., 2008b, Determination of the $\delta\left({ }^{2} \mathrm{H} /{ }^{1} \mathrm{H}\right)$ of water-RSIL lab code 1574 , chap. $\mathrm{C} 1$ of Révész, Kinga, and Coplen, T.B., eds., Methods of the Reston Stable Isotope Laboratory: U.S. Geological Survey Techniques and Methods, book 10, sec. C, chap. 1, $27 \mathrm{p}$.

Rose, P.R., 1972, Edwards group, surface and subsurface, central Texas: Bureau of Economic Geology Report of Investigations, no. 74, 198 p.

Sauer, V.B., and Meyer, R.W., 1992, Determination of error in individual discharge measurements: U.S. Geological Survey Open-File Report 92-144, 21 p., accessed July 23, 2012, at http://pubs.usgs.gov/of/1992/ofr92-144/.

Sharp, J.M., Jr., and Banner, J.L., 1997, The Edwards aquifer-A resource in conflict: Geological Society of America Today, v. 7, p. 1-9.

Slade, R.M., Jr., Bentley, J.T., and Michaud, Dana, 2002, Results of streamflow gain-loss studies in Texas, with emphasis on gains from and losses to major and minor aquifers: U.S. Geological Survey Open-File Report 02-068, $131 \mathrm{p}$.

Trudgill, S.T., 1995, Solute modelling in catchment systems: West Sussex, England, Wiley, 472 p.

Turco, M.J., East, J.W., and Milburn, M.S., 2007, Base flow (1966-2005) and streamflow gain and loss (2006) of the Brazos River, McLennan County to Fort Bend County, Texas: U.S. Geological Survey Scientific Investigations Report 2007-5286, 27 p. 
Turnipseed, D.P., and Sauer, V.B., 2010, Discharge measurements at gaging stations: U.S. Geological Survey Techniques and Methods book 3, chap. A8, 87 p. (Also available at http://pubs.usgs.gov/tm/tm3-a8/.)

U.S. Geological Survey, variously dated, National field manual for the collection of water-quality data: U.S. Geological Survey Techniques of Water-Resources Investigations, book 9, chaps. A1-A9. (Also available at http://pubs.water. usgs.gov/twri9a.)
U.S. Geological Survey, 2012a, USGS water data for Texas: National Water Information System, accessed April 4, 2012, at http://waterdata.usgs.gov/tx/nwis/nwis.

U.S. Geological Survey, 2012b, National hydrography dataset: U.S. Geological Survey, accessed September 29, 2011, at http://nhd.usgs.gov/index.html. 
Appendixes 1-3 

Appendix 1. Quality-assurance hydrologic measurements during the July 2008, August 2009, and March 2010 surveys in the upper Nueces River Basin, south-central Texas. [ft³/s, cubic feet per second; Spring, springflow; --, not collected or not applicable; P, poor; RPD, relative percent difference; \%, percent; SW, surface-water streamflow; G, good; F, fair]

\begin{tabular}{|c|c|c|c|c|c|c|c|c|c|c|}
\hline \multirow{2}{*}{$\begin{array}{l}\text { Site } \\
\text { identifier } \\
\text { (fig. 1) }\end{array}$} & \multirow[b]{2}{*}{ Watershed } & \multirow[b]{2}{*}{$\begin{array}{l}\text { U.S. Geological } \\
\text { Survey station } \\
\text { number }\end{array}$} & \multirow[b]{2}{*}{$\begin{array}{l}\text { U.S. Geological Survey } \\
\text { station name }\end{array}$} & \multirow[b]{2}{*}{$\begin{array}{l}\text { Type } \\
\text { of data }\end{array}$} & \multicolumn{2}{|c|}{ July 21-23, 2008} & \multicolumn{2}{|c|}{ August 8-18, 2009} & \multicolumn{2}{|c|}{ March 22-24, 2010} \\
\hline & & & & & $\begin{array}{l}\text { Measure- } \\
\text { ment } \\
\left(\mathrm{ft}^{3} / \mathrm{s}\right)\end{array}$ & $\begin{array}{l}\text { Measure- } \\
\text { ment } \\
\text { rating }\end{array}$ & $\begin{array}{l}\text { Measure- } \\
\text { ment } \\
\left(\mathrm{ft}^{3} / \mathrm{s}\right)\end{array}$ & $\begin{array}{l}\text { Measure- } \\
\text { ment } \\
\text { rating }\end{array}$ & $\begin{array}{l}\text { Measure- } \\
\text { ment } \\
\left(\mathrm{ft}^{3} / \mathrm{s}\right)\end{array}$ & $\begin{array}{l}\text { Measure- } \\
\text { ment } \\
\text { rating }\end{array}$ \\
\hline \multirow[t]{3}{*}{6} & \multirow{3}{*}{$\begin{array}{l}\text { West Nueces } \\
\text { River }\end{array}$} & \multirow[t]{3}{*}{293913100230301} & \multirow[t]{3}{*}{ Spring 3 on West Nueces River ${ }^{1}$} & Spring & -- & -- & 0.01 & $\mathrm{P}$ & -- & -- \\
\hline & & & & Spring & -- & -- & 0.01 & $\mathrm{P}$ & -- & -- \\
\hline & & & & $R P D$ & & & $0 \%$ & & & \\
\hline \multirow[t]{3}{*}{13} & \multirow[t]{3}{*}{ Nueces River } & \multirow[t]{3}{*}{08189983} & \multirow{3}{*}{$\begin{array}{l}\text { Nueces River downstream from } \\
\text { Hackberry Creek near Vance, } \\
\text { Tex. }\end{array}$} & SW & -- & -- & 9.49 & $\mathrm{P}$ & -- & -- \\
\hline & & & & SW & -- & -- & 9.76 & $\mathrm{P}$ & -- & -- \\
\hline & & & & $R P D$ & & & $3 \%$ & & & \\
\hline \multirow[t]{3}{*}{19} & \multirow[t]{3}{*}{ Nueces River } & \multirow[t]{3}{*}{08189985} & \multirow[t]{3}{*}{ Nueces River at Vance, Tex. } & SW & -- & -- & 2.19 & G & -- & -- \\
\hline & & & & SW & -- & -- & 2.28 & $\mathrm{~F}$ & -- & -- \\
\hline & & & & $R P D$ & & & $4 \%$ & & & \\
\hline \multirow[t]{3}{*}{32} & \multirow[t]{3}{*}{ Nueces River } & \multirow[t]{3}{*}{08189998} & \multirow{3}{*}{$\begin{array}{l}\text { Nueces River at County Road } 414 \\
\text { at Montell, Tex. }\end{array}$} & SW & -- & -- & 0.72 & $\mathrm{~F}$ & -- & -- \\
\hline & & & & SW & -- & -- & 0.70 & $\mathrm{~F}$ & -- & -- \\
\hline & & & & $R P D$ & & & $3 \%$ & & & \\
\hline \multirow[t]{3}{*}{33} & \multirow[t]{3}{*}{ Nueces River } & \multirow[t]{3}{*}{08190000} & \multirow[t]{3}{*}{ Nueces River at Laguna, Tex. } & SW & -- & -- & 18.3 & $\mathrm{~F}$ & -- & -- \\
\hline & & & & SW & -- & -- & 18.5 & $\mathrm{~F}$ & -- & -- \\
\hline & & & & $R P D$ & & & $1 \%$ & & & \\
\hline \multirow[t]{3}{*}{50} & Frio River & 08194800 & East Frio River upstream of Indian & SW & 13.3 & G & -- & -- & -- & -- \\
\hline & & & Creek near Leakey, Tex. & SW & 12.4 & $\mathrm{~F}$ & -- & -- & -- & -- \\
\hline & & & & $R P D$ & $7 \%$ & & & & & \\
\hline 51 & Frio River & 08194840 & Frio River at Leakey, Tex. & SW & -- & -- & 1.03 & $\mathrm{~F}$ & -- & -- \\
\hline & & & & SW & -- & -- & 0.61 & $\mathrm{P}$ & -- & -- \\
\hline & & & & $R P D$ & & & $51 \%$ & & & \\
\hline 61 & Frio River & 08195000 & Frio River at Concan, Tex. & SW & -- & -- & 6.94 & $\mathrm{~F}$ & -- & -- \\
\hline & & & & SW & -- & -- & 6.95 & $\mathrm{~F}$ & -- & -- \\
\hline & & & & $R P D$ & & & $0 \%$ & & & \\
\hline 65 & Sabinal River & 08197910 & Sabinal River downstream from & SW & 2.45 & $\mathrm{~F}$ & -- & -- & -- & -- \\
\hline & & & Marler Creek near Vanderpool, & SW & 2.50 & $\mathrm{~F}$ & -- & -- & -- & -- \\
\hline & & & Tex. & $R P D$ & $2 \%$ & & & & & \\
\hline
\end{tabular}


Appendix 2. Water-quality data collected during the August 2009 and March 2010 surveys in the upper Nueces River Basin, south-central Texas.

$\left[{ }^{\circ} \mathrm{C}\right.$, degrees Celsius; na, not applicable; ENV, environmental sample; --, not analyzed; mg/L, milligrams per liter; LED, light-emitting diode; nm, nanometers; E, estimated; <, less than; $\mu \mathrm{g} / \mathrm{L}$, micrograms per liter]

\begin{tabular}{|c|c|c|c|c|c|c|c|c|c|c|c|}
\hline $\begin{array}{l}\text { Site } \\
\text { identi- } \\
\text { fier }\end{array}$ & Watershed & $\begin{array}{l}\text { U.S. Geological } \\
\text { Survey station } \\
\text { number }\end{array}$ & $\begin{array}{l}\text { State well } \\
\text { number }\end{array}$ & $\begin{array}{l}\text { U.S. Geological Survey } \\
\text { station name }\end{array}$ & Date & Time & $\begin{array}{c}\text { Sample } \\
\text { type }\end{array}$ & $\begin{array}{c}\text { Barometric } \\
\text { pressure, } \\
\text { millimeters } \\
\text { of mercury }\end{array}$ & $\begin{array}{l}\text { Temper- } \\
\text { ature, } \\
\text { air } \\
\left({ }^{\circ} \mathrm{C}\right)\end{array}$ & $\begin{array}{c}\text { Depth to } \\
\text { water level } \\
\text { (feet below } \\
\text { land surface) }\end{array}$ & $\begin{array}{l}\text { Discharge, } \\
\text { instantaneous } \\
\text { (cubic feet } \\
\text { per second) }\end{array}$ \\
\hline 4 & West Nueces River & 294529100234701 & na & Spring 1 on West Nueces River ${ }^{1}$ & $8 / 18 / 2009$ & 1600 & ENV & -- & -- & -- & -- \\
\hline 4 & West Nueces River & 294529100234701 & na & Spring 1 on West Nueces River ${ }^{1}$ & $3 / 23 / 2010$ & 920 & ENV & 717 & 13.4 & -- & -- \\
\hline 5 & West Nueces River & 294443100213701 & na & Spring 2 on West Nueces River ${ }^{1}$ & $8 / 18 / 2009$ & 1330 & ENV & -- & -- & -- & -- \\
\hline 6 & West Nueces River & 293913100230301 & na & Spring 3 on West Nueces River ${ }^{1}$ & $8 / 18 / 2009$ & 1045 & ENV & -- & -- & -- & -- \\
\hline 8 & Nueces River & 08189979 & na & $\begin{array}{l}\text { Hackberry Creek at Ranch Road } 335 \\
\text { near Vance, Tex. }\end{array}$ & $8 / 13 / 2009$ & 1430 & ENV & 715 & -- & -- & -- \\
\hline 8 & Nueces River & 08189979 & na & $\begin{array}{l}\text { Hackberry Creek at Ranch Road } 335 \\
\text { near Vance, Tex. }\end{array}$ & $3 / 23 / 2010$ & 1400 & ENV & 710 & 20.8 & -- & -- \\
\hline 9 & Nueces River & 0818998070 & na & $\begin{array}{l}\text { East Prong Nueces River upstream of } \\
\text { Eagle Ranch near Rocksprings, Tex. }\end{array}$ & $8 / 12 / 2009$ & 1730 & ENV & -- & -- & -- & -- \\
\hline 9 & Nueces River & 0818998070 & na & $\begin{array}{l}\text { East Prong Nueces River upstream of } \\
\text { Eagle Ranch near Rocksprings, Tex. }\end{array}$ & $3 / 23 / 2010$ & 1620 & ENV & 711 & 22.8 & -- & -- \\
\hline 10 & Nueces River & 295702099571801 & na & $\begin{array}{l}\text { Spring on East Prong Nueces River } \\
\text { near Vance, Tex. }{ }^{1}\end{array}$ & $8 / 12 / 2009$ & 1830 & ENV & -- & -- & -- & -- \\
\hline 22 & Nueces River & 0818999010 & na & Nueces River near Barksdale, Tex. & $3 / 23 / 2010$ & 1500 & ENV & 718 & 22.6 & -- & 46 \\
\hline 29 & Nueces River & 0818999640 & na & $\begin{array}{l}\text { Old Faithful Spring near Camp Wood, } \\
\text { Tex. }\end{array}$ & $3 / 24 / 2010$ & 0920 & ENV & 724 & 17.1 & -- & -- \\
\hline 39 & Frio River & 295454099473101 & na & Spring 1 on West Frio River, Tex. ${ }^{1}$ & $8 / 12 / 2009$ & 1630 & ENV & 713 & 22.7 & -- & -- \\
\hline 40 & Frio River & 295144099480201 & na & Spring 2 on West Frio River, Tex. ${ }^{1}$ & $8 / 12 / 2009$ & 1400 & ENV & -- & 28.0 & -- & -- \\
\hline 40 & Frio River & 295144099480201 & na & Spring 2 on West Frio River, Tex. ${ }^{1}$ & $3 / 22 / 2010$ & 1700 & ENV & 716 & -- & -- & -- \\
\hline 42 & Frio River & 08194690 & na & $\begin{array}{l}\text { West Frio River upstream of Kent } \\
\text { Creek near Leakey, Tex. }\end{array}$ & $3 / 22 / 2010$ & 1800 & ENV & 719 & -- & -- & 7.7 \\
\hline 46 & Frio River & 295100099403001 & na & Spring on East Frio River, Tex. ${ }^{1}$ & $8 / 13 / 2009$ & 1100 & ENV & 715 & 22.5 & -- & -- \\
\hline 53 & Frio River & 08194850 & na & Leakey Spring Run at Leakey, Tex. & $8 / 12 / 2009$ & 1430 & ENV & -- & 36.0 & -- & -- \\
\hline 53 & Frio River & 08194850 & na & Leakey Spring Run at Leakey, Tex. & $3 / 22 / 2010$ & 1430 & ENV & 727 & 21.2 & -- & -- \\
\hline 61 & Frio River & 08195000 & na & Frio River at Concan, Tex. & $3 / 22 / 2010$ & 1230 & ENV & 737 & 15.4 & -- & -- \\
\hline 62 & Sabinal River & 294950099353801 & na & AS-69-12-401 & $8 / 13 / 2009$ & 1615 & ENV & -- & -- & -- & -- \\
\hline 62 & Sabinal River & 294950099353801 & na & AS-69-12-401 & $3 / 24 / 2010$ & 1400 & ENV & 711 & -- & -- & -- \\
\hline 64 & Sabinal River & 08197907 & na & $\begin{array}{l}\text { Sabinal River downstream from } \\
\text { Wedgeworth Creek near Vander- } \\
\text { pool, Tex. }\end{array}$ & $8 / 14 / 2009$ & 1000 & ENV & 718 & 25.0 & -- & -- \\
\hline 64 & Sabinal River & 08197907 & na & $\begin{array}{l}\text { Sabinal River downstream from } \\
\text { Wedgeworth Creek near Vander- } \\
\text { pool, Tex. }\end{array}$ & $3 / 24 / 2010$ & 1300 & ENV & 717 & -- & -- & -- \\
\hline 72 & Sabinal River & 08197970 & na & Sabinal River at Utopia, Tex. & $3 / 24 / 2010$ & 1600 & ENV & 728 & 20.1 & -- & -- \\
\hline 75 & West Nueces River & 293551100281701 & RP-70-29-101 & RP-70-29-101 (Kickapoo Caverns) & $8 / 17 / 2009$ & 1540 & ENV & 722 & -- & 237.05 & -- \\
\hline 75 & West Nueces River & 293551100281701 & RP-70-29-101 & RP-70-29-101 (Kickapoo Caverns) & $3 / 23 / 2010$ & 1100 & ENV & -- & -- & 245.34 & -- \\
\hline 76 & Nueces River & 300259100030801 & JJ-55-64-503 & JJ-55-64-503 (Lazy H Ranch) & $8 / 13 / 2009$ & 1200 & ENV & 705 & -- & 300.35 & -- \\
\hline 77 & Sabinal River & 294815099343801 & AS-69-12-501 & AS-69-12-501 & $8 / 13 / 2009$ & 1530 & ENV & -- & -- & -- & -- \\
\hline
\end{tabular}


Appendix 2. Water-quality data collected during the August 2009 and March 2010 surveys in the upper Nueces River Basin, south-central Texas.-Continued

$\left[{ }^{\circ} \mathrm{C}\right.$, degrees Celsius; na, not applicable; ENV, environmental sample; --, not analyzed; mg/L, milligrams per liter; LED, light-emitting diode; nm, nanometers; E, estimated; <, less than; $\mu \mathrm{g} / \mathrm{L}$, micrograms per liter]

\begin{tabular}{|c|c|c|c|c|c|c|c|c|c|c|c|c|c|c|c|}
\hline $\begin{array}{l}\text { Site } \\
\text { identi- } \\
\text { fier }\end{array}$ & Date & $\begin{array}{c}\text { Dis- } \\
\text { solved } \\
\text { oxygen, } \\
\text { water, } \\
\text { unfil- } \\
\text { tered } \\
\text { (mg/L) }\end{array}$ & $\begin{array}{c}\text { Dissolved } \\
\text { oxygen, } \\
\text { water, } \\
\text { unfiltered } \\
\text { (percent of } \\
\text { saturation) }\end{array}$ & $\begin{array}{l}\text { pH, } \\
\text { water, } \\
\text { unfil- } \\
\text { tered, } \\
\text { field } \\
\text { (stan- } \\
\text { dard } \\
\text { units) }\end{array}$ & $\begin{array}{c}\text { pH, } \\
\text { water, } \\
\text { unfiltered, } \\
\text { labora- } \\
\text { tory } \\
\text { (standard } \\
\text { units) }\end{array}$ & $\begin{array}{c}\text { Specific } \\
\text { conduc- } \\
\text { tance, } \\
\text { water, } \\
\text { unfiltered, } \\
\text { laboratory } \\
\text { (microsie- } \\
\text { mens per } \\
\text { centimeter } \\
\text { at } 25^{\circ} \mathrm{C} \text { ) }\end{array}$ & $\begin{array}{l}\text { Specific } \\
\text { conduc- } \\
\text { tance, } \\
\text { water, } \\
\text { unfiltered } \\
\text { (microsie- } \\
\text { mens per } \\
\text { centimeter } \\
\text { at } 25^{\circ} \mathrm{C} \text { ) }\end{array}$ & $\begin{array}{c}\text { Temper- } \\
\text { ature, } \\
\text { water } \\
\left({ }^{\circ} \mathrm{C}\right)\end{array}$ & $\begin{array}{l}\text { Turbidity, water, } \\
\text { unfiltered, } \\
\text { monochrome } \\
\text { near infrared LED } \\
\text { light, 780-900 nm, } \\
\text { detection angle } \\
90+/-2.5 \text { degrees, } \\
\text { formazin nephelo- } \\
\text { metric units } \\
\text { (FNU) }\end{array}$ & $\begin{array}{c}\text { Dissolved } \\
\text { solids } \\
\text { dried at } \\
180^{\circ} \mathrm{C}, \\
\text { water, } \\
\text { filtered } \\
\text { (mg/L) }\end{array}$ & $\begin{array}{c}\text { Hardness, } \\
\text { water } \\
\text { (mg/L as } \\
\mathrm{CaCO}_{3} \text { ) }\end{array}$ & $\begin{array}{l}\text { Noncarbon- } \\
\text { ate hard- } \\
\text { ness, water, } \\
\text { filtered, field } \\
\text { (milligrams } \\
\text { per liter as } \\
\text { calcium } \\
\text { carbonate) }\end{array}$ & $\begin{array}{c}\text { Calcium, } \\
\text { water, } \\
\text { filtered } \\
\text { (mg/L) }\end{array}$ & $\begin{array}{l}\text { Magne- } \\
\text { sium, wa- } \\
\text { ter, filtered } \\
\text { (mg/L) }\end{array}$ & $\begin{array}{c}\text { Potassium, } \\
\text { water, } \\
\text { filtered } \\
\text { (mg/L) }\end{array}$ \\
\hline 4 & $8 / 18 / 2009$ & 7.8 & -- & E7.0 & 7.8 & 373 & 366 & 23.2 & 1.5 & 214 & 178 & 4 & 58.6 & 7.57 & 0.52 \\
\hline 4 & $3 / 23 / 2010$ & 8.5 & 100 & 7.9 & 7.9 & 360 & 379 & 20.4 & -- & 213 & 178 & -- & 59.1 & 7.34 & 0.50 \\
\hline 5 & $8 / 18 / 2009$ & 7.2 & -- & 7.2 & 7.6 & 504 & 499 & 21.7 & $<1.0$ & 282 & 264 & 21 & 74.8 & 18.7 & 0.40 \\
\hline 6 & $8 / 18 / 2009$ & -- & -- & -- & -- & -- & -- & -- & -- & -- & -- & -- & -- & -- & -- \\
\hline 8 & $8 / 13 / 2009$ & 6.6 & 80 & 6.9 & 7.6 & 411 & 410 & 21.4 & $<1.0$ & 236 & 209 & -- & 62.5 & 12.8 & 0.83 \\
\hline 8 & $3 / 23 / 2010$ & 7.6 & 90 & 7.4 & 7.7 & 394 & 405 & 20.4 & -- & 221 & 200 & -- & 58.7 & 12.9 & 0.85 \\
\hline 9 & $8 / 12 / 2009$ & 10.9 & -- & 7.8 & 8.0 & 398 & 400 & 25.0 & -- & 228 & 196 & 6 & 59.4 & 11.6 & 0.63 \\
\hline 9 & $3 / 23 / 2010$ & 11.3 & 138 & 8.4 & 8.2 & 372 & 383 & 21.8 & -- & 213 & 191 & -- & 57.0 & 11.8 & 0.66 \\
\hline 10 & $8 / 12 / 2009$ & 8.1 & -- & E7.4 & 7.7 & 428 & 420 & 20.5 & -- & 244 & 201 & -- & 59.3 & 12.8 & 0.61 \\
\hline 22 & $3 / 23 / 2010$ & 10.4 & 119 & 8.1 & 8.2 & 353 & 366 & 18.9 & -- & 206 & 178 & -- & 49.3 & 13.3 & 0.67 \\
\hline 29 & $3 / 24 / 2010$ & 8.2 & 91 & 7.5 & 7.9 & 375 & 375 & 18.1 & -- & 203 & 185 & -- & 54.6 & 11.7 & 0.55 \\
\hline 39 & $8 / 12 / 2009$ & 8.1 & 96 & E7.2 & 7.7 & 412 & 405 & 20.5 & $<1.0$ & 231 & 189 & -- & 61.3 & 8.75 & 0.44 \\
\hline 40 & $8 / 12 / 2009$ & 8.2 & -- & E7.3 & 7.8 & 427 & 421 & 21.0 & -- & 242 & 208 & 1 & 64.3 & 11.5 & 0.52 \\
\hline 40 & $3 / 22 / 2010$ & 7.9 & 93 & 7.8 & 7.9 & 401 & 424 & 20.0 & -- & 229 & 208 & -- & 63.5 & 12.1 & 0.53 \\
\hline 42 & $3 / 22 / 2010$ & 9.9 & 108 & 8.4 & 8.2 & 311 & 317 & 16.8 & -- & 182 & 156 & 6 & 41.3 & 12.9 & 0.65 \\
\hline 46 & $8 / 13 / 2009$ & 8.2 & 97 & 7.5 & 7.8 & 418 & 416 & 20.4 & 2.2 & 235 & 214 & 15 & 63.4 & 13.6 & 0.59 \\
\hline 52 & $8 / 12 / 2009$ & 7.3 & -- & 7.1 & 7.6 & 451 & 443 & 22.0 & $<1.0$ & 245 & 212 & -- & 63.4 & 12.9 & 0.77 \\
\hline 52 & $3 / 22 / 2010$ & 7.7 & 85 & 7.9 & 7.9 & 396 & 415 & 18.1 & -- & 219 & 205 & 22 & 60.3 & 13.1 & 0.79 \\
\hline 61 & $3 / 22 / 2010$ & 10.2 & 104 & 7.9 & 8.2 & 439 & 446 & 14.6 & -- & 265 & 215 & 31 & 61.4 & 14.8 & 0.77 \\
\hline 62 & $8 / 13 / 2009$ & 7.7 & -- & 7.1 & 7.7 & 466 & 467 & 19.9 & $<1.0$ & 267 & 228 & -- & 63.9 & 16.6 & 0.45 \\
\hline 62 & $3 / 24 / 2010$ & 9.0 & 99 & 7.5 & 7.7 & 462 & 480 & 16.7 & -- & 268 & 249 & -- & 70.6 & 17.5 & 0.55 \\
\hline 64 & $8 / 14 / 2009$ & 6.3 & 84 & 8.0 & 8.0 & 397 & 402 & 26.8 & $<1.0$ & 226 & 204 & 6 & 54.9 & 16.3 & 0.53 \\
\hline 64 & $3 / 24 / 2010$ & 9.2 & 99 & 7.9 & 8.2 & 421 & 437 & 16.4 & -- & 257 & 226 & -- & 59.2 & 18.9 & 0.60 \\
\hline 72 & $3 / 24 / 2010$ & 9.7 & 111 & 7.8 & 8.1 & 509 & 530 & 19.4 & -- & 283 & 273 & -- & 85.3 & 14.5 & 0.93 \\
\hline 75 & $8 / 17 / 2009$ & 6.7 & 84 & 7.3 & 7.6 & 384 & 383 & 23.8 & 5.3 & 226 & 187 & -- & 64.9 & 6.04 & 0.67 \\
\hline 75 & $3 / 23 / 2010$ & 7.1 & -- & 7.9 & 7.7 & 373 & 383 & 23.5 & -- & 209 & 188 & -- & 65.1 & 6.23 & 0.70 \\
\hline 76 & $8 / 13 / 2009$ & 6.7 & 85 & E7.3 & 7.8 & 410 & 413 & 23.2 & 2.0 & 231 & 199 & -- & 51.8 & 16.8 & 0.63 \\
\hline 77 & $8 / 13 / 2009$ & 0.2 & -- & E7.0 & 7.5 & 1,180 & 1,190 & 25.2 & -- & 808 & 515 & 242 & 95.1 & 65.3 & 20.3 \\
\hline
\end{tabular}


Appendix 2. Water-quality data collected during the August 2009 and March 2010 surveys in the upper Nueces River Basin, south-central Texas.-Continued

$\left[{ }^{\circ} \mathrm{C}\right.$, degrees Celsius; na, not applicable; ENV, environmental sample; --, not analyzed; mg/L, milligrams per liter; LED, light-emitting diode; nm, nanometers; E, estimated; <, less than; $\mu \mathrm{g} / \mathrm{L}$, micrograms per liter]

\begin{tabular}{|c|c|c|c|c|c|c|c|c|c|c|c|c|c|c|c|}
\hline $\begin{array}{l}\text { Site } \\
\text { iden- } \\
\text { tifier }\end{array}$ & Date & $\begin{array}{c}\text { Sodium } \\
\text { adsorp- } \\
\text { tion } \\
\text { ratio, } \\
\text { water, } \\
\text { number }\end{array}$ & $\begin{array}{l}\text { Sodium } \\
\text { fraction of } \\
\text { cations, } \\
\text { water, } \\
\text { percent in } \\
\text { equiva- } \\
\text { lents of } \\
\text { major } \\
\text { cations }\end{array}$ & $\begin{array}{c}\text { Sodium, } \\
\text { water, } \\
\text { filtered } \\
\text { (mg/L) }\end{array}$ & $\begin{array}{c}\text { Alkalinity, } \\
\text { water, } \\
\text { filtered, } \\
\text { fixed end- } \\
\text { point } \\
\text { (pH 4.5) } \\
\text { titration, } \\
\text { field } \\
\text { (mg/L as } \\
\mathrm{CaCO}_{3} \text { ) }\end{array}$ & $\begin{array}{c}\text { Alkalinity, } \\
\text { water, filtered, } \\
\text { inflection-point } \\
\text { titration method } \\
\text { (incremental } \\
\text { titration } \\
\text { method), field } \\
\text { (mg/L as } \mathrm{CaCO}_{3} \text { ) }\end{array}$ & $\begin{array}{c}\text { Bicarbonate, } \\
\text { water, filtered, } \\
\text { inflection- } \\
\text { point titration } \\
\text { method } \\
\text { (incremental } \\
\text { titration } \\
\text { method), field } \\
\text { (mg/L) }\end{array}$ & $\begin{array}{c}\text { Bro- } \\
\text { mide, } \\
\text { water, } \\
\text { filtered } \\
\text { (mg/L) }\end{array}$ & $\begin{array}{c}\text { Carbon } \\
\text { diox- } \\
\text { ide, } \\
\text { water, } \\
\text { unfil- } \\
\text { tered } \\
\text { (mg/L) }\end{array}$ & $\begin{array}{c}\text { Carbonate, } \\
\text { water, filtered, } \\
\text { inflection-point } \\
\text { titration method } \\
\text { (incremental } \\
\text { titration } \\
\text { method), } \\
\text { field } \\
\text { (mg/L) }\end{array}$ & $\begin{array}{c}\text { Chlo- } \\
\text { ride, } \\
\text { water, } \\
\text { filtered } \\
(\mathrm{mg} / \mathrm{L})\end{array}$ & $\begin{array}{c}\text { Fluo- } \\
\text { ride, } \\
\text { water, } \\
\text { filtered } \\
\text { (mg/L) }\end{array}$ & $\begin{array}{c}\text { Hydrogen } \\
\text { ion, water, } \\
\text { unfiltered, } \\
\text { calculated } \\
\text { (mg/L) }\end{array}$ & $\begin{array}{c}\text { Silica, } \\
\text { water, } \\
\text { filtered } \\
(\mathrm{mg} / \mathrm{L} \text { as } \\
\left.\mathrm{SiO}_{2}\right)\end{array}$ & $\begin{array}{c}\text { Sulfate, } \\
\text { water, } \\
\text { filtered } \\
\text { (mg/L) }\end{array}$ \\
\hline 4 & $8 / 18 / 2009$ & 0.17 & 6 & 5.26 & -- & 174 & 212 & 0.06 & E34 & $<1$ & 9.85 & 0.14 & E0.00010 & 11.5 & 5.47 \\
\hline 4 & $3 / 23 / 2010$ & 0.16 & 6 & 5.02 & -- & 210 & 255 & 0.06 & 5.7 & $<1$ & 10.5 & 0.19 & 0.00001 & 11.3 & 5.50 \\
\hline 5 & $8 / 18 / 2009$ & 0.15 & 4 & 5.52 & -- & 243 & 296 & 0.07 & 28 & $<1$ & 10.5 & E0.10 & 0.00006 & 11.1 & 7.16 \\
\hline 6 & $8 / 18 / 2009$ & -- & -- & -- & -- & -- & -- & -- & -- & -- & -- & -- & -- & -- & -- \\
\hline 8 & $8 / 13 / 2009$ & 0.15 & 5 & 4.91 & -- & -- & -- & 0.06 & -- & -- & 9.15 & 0.15 & 0.00012 & 11.8 & 5.61 \\
\hline 8 & $3 / 23 / 2010$ & 0.14 & 5 & 4.51 & 186 & -- & -- & 0.05 & 14 & -- & 9.50 & 0.18 & 0.00004 & 11.9 & 5.55 \\
\hline 9 & $8 / 12 / 2009$ & 0.17 & 6 & 5.57 & -- & 191 & 232 & 0.06 & 6.0 & $<1$ & 10.2 & 0.16 & 0.00002 & 11.0 & 6.05 \\
\hline 9 & $3 / 23 / 2010$ & 0.16 & 6 & 5.13 & 184 & -- & -- & 0.06 & 1.5 & -- & 9.99 & 0.19 & 0.00000 & 10.8 & 6.05 \\
\hline 10 & $8 / 12 / 2009$ & 0.15 & 5 & 4.79 & -- & 206 & 250 & 0.07 & E16 & $<1$ & 10.1 & 0.10 & E0.00004 & 11.7 & 5.97 \\
\hline 22 & $3 / 23 / 2010$ & 0.18 & 6 & 5.50 & 170 & -- & -- & 0.05 & 2.6 & -- & 12.1 & 0.18 & 0.00001 & 10.6 & 10.2 \\
\hline 29 & $3 / 24 / 2010$ & 0.18 & 6 & 5.47 & 177 & -- & -- & 0.06 & 10 & -- & 11.8 & 0.11 & 0.00003 & 10.7 & 8.11 \\
\hline 39 & $8 / 12 / 2009$ & 0.14 & 5 & 4.26 & -- & 192 & 233 & 0.07 & E27 & $<1$ & 9.34 & E.07 & E0.00007 & 10.2 & 5.99 \\
\hline 40 & $8 / 12 / 2009$ & 0.15 & 5 & 4.89 & -- & 208 & 252 & 0.07 & E18 & $<1$ & 10.6 & E.06 & E0.00004 & 11.0 & 5.89 \\
\hline 40 & $3 / 22 / 2010$ & 0.15 & 5 & 4.88 & -- & 210 & 255 & 0.06 & 7.3 & E1 & 11.2 & 0.15 & 0.00002 & 10.8 & 6.10 \\
\hline 42 & $3 / 22 / 2010$ & 0.18 & 7 & 5.05 & -- & 151 & 183 & 0.04 & 1.1 & $<1$ & 10.6 & 0.18 & 0.00000 & 9.16 & 7.90 \\
\hline 46 & $8 / 13 / 2009$ & 0.14 & 5 & 4.81 & -- & 201 & 244 & 0.07 & 13 & $<1$ & 9.07 & E0.09 & 0.00003 & 10.9 & 5.68 \\
\hline 52 & $8 / 12 / 2009$ & 0.17 & 6 & 5.68 & -- & 213 & 259 & 0.07 & 33 & $<1$ & 11.3 & E0.09 & 0.00008 & 11.2 & 10.3 \\
\hline 52 & $3 / 22 / 2010$ & 0.17 & 6 & 5.51 & -- & 184 & 224 & 0.05 & 5.0 & $<1$ & 12.3 & 0.16 & 0.00001 & 10.5 & 14.0 \\
\hline 61 & $3 / 22 / 2010$ & 0.19 & 6 & 6.49 & -- & 185 & 225 & 0.04 & 4.7 & $<1$ & 13.5 & 0.18 & 0.00001 & 10.4 & 28.5 \\
\hline 62 & $8 / 13 / 2009$ & 0.14 & 4 & 4.67 & -- & 261 & 318 & 0.06 & 38 & $<1$ & 9.33 & 0.12 & 0.00008 & 11.3 & 5.83 \\
\hline 62 & $3 / 24 / 2010$ & 0.14 & 4 & 4.92 & 226 & -- & -- & 0.05 & 15 & -- & 10.7 & 0.16 & 0.00003 & 11.2 & 6.74 \\
\hline 64 & $8 / 14 / 2009$ & 0.18 & 6 & 5.87 & -- & 198 & 239 & 0.08 & 4.3 & 1 & 10.5 & E0.09 & 0.00001 & 13.7 & 5.18 \\
\hline 64 & $3 / 24 / 2010$ & 0.17 & 5 & 5.83 & 215 & -- & -- & 0.03 & 5.8 & -- & 12.1 & 0.15 & 0.00001 & 9.78 & 10.9 \\
\hline 72 & $3 / 24 / 2010$ & 0.17 & 5 & 6.45 & 204 & -- & -- & 0.05 & 6.9 & -- & 13.0 & 0.19 & 0.00002 & 11.7 & 42.0 \\
\hline 75 & $8 / 17 / 2009$ & 0.15 & 5 & 4.55 & -- & 189 & 230 & 0.06 & 19 & $<1$ & 8.82 & E.08 & 0.00005 & 10.8 & 4.99 \\
\hline 75 & $3 / 23 / 2010$ & 0.15 & 5 & 4.60 & 174 & -- & -- & 0.06 & 4.1 & -- & 9.38 & 0.14 & 0.00001 & 11.3 & 5.26 \\
\hline 76 & $8 / 13 / 2009$ & 0.17 & 6 & 5.63 & -- & 201 & 244 & 0.06 & E20 & $<1$ & 10.8 & 0.21 & E0.00005 & 11.9 & 5.53 \\
\hline 77 & $8 / 13 / 2009$ & 1.04 & 18 & 53.9 & -- & 273 & 332 & 0.25 & E54 & $<1$ & 33.3 & 2.85 & E0.00010 & 11.3 & 326 \\
\hline
\end{tabular}


Appendix 2. Water-quality data collected during the August 2009 and March 2010 surveys in the upper Nueces River Basin, south-central Texas.-Continued

$\left[{ }^{\circ} \mathrm{C}\right.$, degrees Celsius; na, not applicable; ENV, environmental sample; --, not analyzed; mg/L, milligrams per liter; LED, light-emitting diode; nm, nanometers; E, estimated; <, less than; $\mu \mathrm{g} / \mathrm{L}$, micrograms per liter]

\begin{tabular}{|c|c|c|c|c|c|c|c|c|c|c|c|c|c|c|}
\hline $\begin{array}{l}\text { Site } \\
\text { iden- } \\
\text { tifier }\end{array}$ & Date & $\begin{array}{l}\text { Aluminum, } \\
\text { water, } \\
\text { filtered } \\
(\mu \mathrm{g} / \mathrm{L})\end{array}$ & $\begin{array}{c}\text { Barium, } \\
\text { water, } \\
\text { filtered } \\
(\mu \mathrm{g} / \mathrm{L})\end{array}$ & $\begin{array}{c}\text { Beryllium, } \\
\text { water, } \\
\text { filtered } \\
(\mu \mathrm{g} / \mathrm{L})\end{array}$ & $\begin{array}{c}\text { Cadmium, } \\
\text { water, } \\
\text { filtered } \\
\text { ( } \mu \mathrm{g} / \mathrm{L})\end{array}$ & $\begin{array}{c}\text { Chromium, } \\
\text { water, } \\
\text { filtered } \\
(\mu \mathrm{g} / \mathrm{L})\end{array}$ & $\begin{array}{c}\text { Cobalt, } \\
\text { water, } \\
\text { filtered } \\
(\mu \mathrm{g} / \mathrm{L})\end{array}$ & $\begin{array}{c}\text { Copper, } \\
\text { water, } \\
\text { filtered } \\
(\mu \mathrm{g} / \mathrm{L})\end{array}$ & $\begin{array}{c}\text { Iron, } \\
\text { water, } \\
\text { filtered } \\
(\mu \mathrm{g} / \mathrm{L})\end{array}$ & $\begin{array}{c}\text { Lead, } \\
\text { water, } \\
\text { filtered } \\
(\mu \mathrm{g} / \mathrm{L})\end{array}$ & $\begin{array}{c}\text { Lithium, } \\
\text { water, } \\
\text { filtered } \\
(\mu \mathrm{g} / \mathrm{L})\end{array}$ & $\begin{array}{c}\text { Manganese, } \\
\text { water, } \\
\text { filtered } \\
(\mu \mathrm{g} / \mathrm{L})\end{array}$ & $\begin{array}{l}\text { Molybdenum, } \\
\text { water, } \\
\text { filtered } \\
(\mu \mathrm{g} / \mathrm{L})\end{array}$ & $\begin{array}{c}\text { Nickel, } \\
\text { water, } \\
\text { filtered } \\
(\mu \mathrm{g} / \mathrm{L})\end{array}$ \\
\hline 4 & $8 / 18 / 2009$ & -- & -- & -- & -- & -- & -- & -- & $<4$ & -- & -- & E0.16 & -- & -- \\
\hline 4 & $3 / 23 / 2010$ & $<3.4$ & 54 & $<0.01$ & $<0.02$ & $<0.12$ & 0.37 & $<1$ & E3 & $<0.03$ & 1.3 & 0.9 & 0.5 & 0.38 \\
\hline 5 & $8 / 18 / 2009$ & -- & -- & -- & -- & -- & -- & -- & $<4$ & -- & -- & E.13 & -- & -- \\
\hline 6 & $8 / 18 / 2009$ & -- & -- & -- & -- & -- & -- & -- & -- & -- & -- & -- & -- & -- \\
\hline 8 & $8 / 13 / 2009$ & -- & -- & -- & -- & -- & -- & -- & 5 & -- & -- & 0.4 & -- & -- \\
\hline 8 & $3 / 23 / 2010$ & $<3.4$ & 108 & $<0.01$ & $<0.02$ & 0.12 & 0.42 & $<1$ & $<6$ & $<0.03$ & 1.9 & 0.8 & 0.5 & 0.42 \\
\hline 9 & $8 / 12 / 2009$ & -- & -- & -- & -- & -- & -- & -- & 4 & & & 2.0 & -- & -- \\
\hline 9 & $3 / 23 / 2010$ & $<3.4$ & 75 & $<0.01$ & $<0.02$ & 0.12 & 0.43 & $<1$ & $<6$ & $<0.03$ & 1.9 & 1.0 & 0.6 & 0.39 \\
\hline 10 & $8 / 12 / 2009$ & -- & -- & -- & -- & -- & -- & -- & E3 & -- & -- & 0.6 & -- & -- \\
\hline 22 & $3 / 23 / 2010$ & -- & -- & -- & -- & -- & -- & -- & $<6$ & -- & -- & 1.4 & -- & -- \\
\hline 29 & $3 / 24 / 2010$ & $<3.4$ & 33 & $<0.01$ & $<0.02$ & $<0.12$ & 0.25 & $<1$ & $<6$ & $<0.03$ & 1.3 & 0.5 & 0.5 & 0.34 \\
\hline 39 & $8 / 12 / 2009$ & -- & -- & -- & -- & -- & -- & -- & E2 & -- & -- & 0.4 & -- & -- \\
\hline 40 & $8 / 12 / 2009$ & -- & -- & -- & -- & -- & -- & -- & E2 & -- & -- & E0.19 & -- & -- \\
\hline 40 & $3 / 22 / 2010$ & $<3.4$ & 27 & $<0.01$ & $<0.02$ & $<0.12$ & 0.48 & $<1$ & $<6$ & $<0.03$ & 1.3 & 0.8 & 0.5 & 0.48 \\
\hline 42 & $3 / 22 / 2010$ & $<3.4$ & 27 & $<0.01$ & E0.01 & $<0.12$ & 0.35 & $<1$ & E4 & $<0.03$ & 1.5 & 1.2 & 0.7 & 0.36 \\
\hline 46 & $8 / 13 / 2009$ & -- & -- & -- & -- & -- & -- & -- & $<4$ & -- & -- & 0.3 & -- & -- \\
\hline 52 & $8 / 12 / 2009$ & -- & -- & -- & -- & -- & -- & -- & E3 & -- & -- & 0.8 & -- & -- \\
\hline 52 & $3 / 22 / 2010$ & E2.2 & 29 & $<0.01$ & $<0.02$ & $<0.12$ & 0.34 & E0.59 & $<6$ & $<0.03$ & 1.6 & 0.7 & 0.8 & 0.39 \\
\hline 61 & $3 / 22 / 2010$ & $<3.4$ & 33 & $<0.01$ & $<0.02$ & $<0.12$ & 0.32 & $<1$ & $<6$ & $<0.03$ & 2.2 & 0.8 & 0.7 & 0.42 \\
\hline 62 & $8 / 13 / 2009$ & -- & -- & -- & -- & -- & -- & -- & E2 & -- & -- & 0.5 & -- & -- \\
\hline 62 & $3 / 24 / 2010$ & E3.1 & 25 & $<0.01$ & $<0.02$ & $<0.12$ & 0.16 & $<1$ & $<6$ & $<0.03$ & 1.1 & E0.23 & 0.4 & 0.42 \\
\hline 64 & $8 / 14 / 2009$ & -- & -- & -- & -- & -- & -- & -- & 13 & -- & -- & 8.5 & -- & -- \\
\hline 64 & $3 / 24 / 2010$ & -- & -- & -- & -- & -- & -- & -- & $<6$ & -- & -- & 0.8 & -- & -- \\
\hline 72 & $3 / 24 / 2010$ & $<3.4$ & 38 & $<0.01$ & $<0.02$ & $<0.12$ & 0.32 & $<1$ & $<6$ & $<0.03$ & 2.3 & 1.8 & 0.7 & 0.49 \\
\hline 75 & $8 / 17 / 2009$ & -- & -- & -- & -- & -- & -- & -- & 4 & -- & -- & 1.4 & -- & -- \\
\hline 75 & $3 / 23 / 2010$ & E2.3 & 124 & $<0.01$ & 0.07 & 0.13 & 1.2 & 0.95 & 10 & 0.06 & 1.5 & 3.8 & 0.5 & 0.56 \\
\hline 76 & $8 / 13 / 2009$ & -- & -- & -- & -- & -- & -- & -- & 5 & -- & -- & 0.8 & -- & -- \\
\hline 77 & $8 / 13 / 2009$ & -- & -- & -- & -- & -- & -- & -- & 729 & -- & -- & 31.1 & -- & -- \\
\hline
\end{tabular}


Appendix 2. Water-quality data collected during the August 2009 and March 2010 surveys in the upper Nueces River Basin, south-central Texas.—Continued

$\left[{ }^{\circ} \mathrm{C}\right.$, degrees Celsius; na, not applicable; ENV, environmental sample; --, not analyzed; mg/L, milligrams per liter; LED, light-emitting diode; nm, nanometers; E, estimated; <, less than; $\mu \mathrm{g} / \mathrm{L}$, micrograms per liter]

\begin{tabular}{|c|c|c|c|c|c|c|c|c|c|c|c|c|c|c|}
\hline $\begin{array}{l}\text { Site } \\
\text { identi- } \\
\text { fier }\end{array}$ & Date & $\begin{array}{c}\text { Silver, } \\
\text { water, } \\
\text { filtered } \\
(\mu \mathrm{g} / \mathrm{L})\end{array}$ & $\begin{array}{c}\text { Strontium, } \\
\text { water, } \\
\text { filtered } \\
(\mu \mathrm{g} / \mathrm{L})\end{array}$ & $\begin{array}{c}\text { Thallium, } \\
\text { water, } \\
\text { filtered } \\
(\mu \mathrm{g} / \mathrm{L})\end{array}$ & $\begin{array}{c}\text { Vanadium, } \\
\text { water, } \\
\text { filtered } \\
(\mu \mathrm{g} / \mathrm{L})\end{array}$ & $\begin{array}{c}\text { Zinc, } \\
\text { water, } \\
\text { filtered } \\
(\mu \mathrm{g} / \mathrm{L})\end{array}$ & $\begin{array}{c}\text { Antimony, } \\
\text { water, } \\
\text { filtered } \\
(\mu \mathrm{g} / \mathrm{L})\end{array}$ & $\begin{array}{c}\text { Arsenic, } \\
\text { water, } \\
\text { filtered } \\
(\mu \mathrm{g} / \mathrm{L})\end{array}$ & $\begin{array}{c}\text { Boron, } \\
\text { water, } \\
\text { filtered } \\
(\mu \mathrm{g} / \mathrm{L})\end{array}$ & $\begin{array}{c}\text { Selenium, } \\
\text { water, } \\
\text { filtered } \\
(\mu \mathrm{g} / \mathrm{L})\end{array}$ & $\begin{array}{c}\text { Uranium } \\
\text { (natural), } \\
\text { water, } \\
\text { filtered } \\
(\mu \mathrm{g} / \mathrm{L})\end{array}$ & $\begin{array}{c}\text { Deuterium/ } \\
\text { protium } \\
\text { ratio, } \\
\text { water, } \\
\text { unfiltered } \\
\text { (per mil) }\end{array}$ & $\begin{array}{c}{ }^{18} 0 /{ }^{16} 0 \\
\text { ratio, } \\
\text { water, } \\
\text { unfiltered } \\
\text { (per mil) }\end{array}$ & $\begin{array}{c}{ }^{87} \mathrm{Sr} /{ }^{86} \mathrm{Sr} \\
\text { ratio, } \\
\text { water, } \\
\text { filtered } \\
\text { (dimen- } \\
\text { sionless) }\end{array}$ \\
\hline 4 & $8 / 18 / 2009$ & -- & 83.0 & -- & -- & -- & -- & -- & -- & -- & -- & -- & -- & 0.70847 \\
\hline 4 & $3 / 23 / 2010$ & $<0.01$ & 78.0 & $<0.02$ & 3.9 & $<2.8$ & 0.10 & 0.49 & 30 & 0.49 & 0.59 & -29.10 & -4.95 & 0.70852 \\
\hline 5 & $8 / 18 / 2009$ & -- & 66.0 & -- & -- & -- & -- & -- & -- & -- & -- & -- & -- & 0.70850 \\
\hline 6 & $8 / 18 / 2009$ & -- & 73.0 & -- & -- & -- & -- & -- & -- & -- & -- & -- & -- & 0.70843 \\
\hline 8 & $8 / 13 / 2009$ & -- & 159 & -- & -- & -- & -- & -- & -- & -- & -- & -- & -- & 0.70813 \\
\hline 8 & $3 / 23 / 2010$ & $<0.01$ & 140 & E0.01 & 4.5 & $<2.8$ & 0.12 & 0.60 & 32 & 0.41 & 0.60 & -30.70 & -5.29 & 0.70819 \\
\hline 9 & $8 / 12 / 2009$ & -- & 224 & -- & -- & -- & -- & -- & -- & -- & -- & -- & -- & 0.70810 \\
\hline 9 & $3 / 23 / 2010$ & $<0.01$ & 192 & $<0.02$ & 4.6 & $<2.8$ & 0.16 & 0.48 & 31 & 0.41 & 0.63 & -29.70 & -5.13 & 0.70808 \\
\hline 10 & $8 / 12 / 2009$ & -- & 188 & -- & -- & -- & -- & -- & -- & -- & -- & -- & -- & 0.70809 \\
\hline 22 & $3 / 23 / 2010$ & -- & -- & -- & -- & -- & -- & -- & -- & -- & -- & -24.80 & -4.16 & 0.70801 \\
\hline 29 & $3 / 24 / 2010$ & $<0.01$ & 155 & $<0.02$ & 2.5 & $<2.8$ & 0.09 & 0.45 & 34 & 0.52 & 0.57 & -26.50 & -4.65 & 0.70795 \\
\hline 39 & $8 / 12 / 2009$ & -- & 103 & -- & -- & -- & -- & -- & -- & -- & -- & -- & -- & 0.70822 \\
\hline 40 & $8 / 12 / 2009$ & -- & 95.0 & -- & -- & -- & -- & -- & -- & -- & -- & -- & -- & 0.70820 \\
\hline 40 & $3 / 22 / 2010$ & $<0.01$ & 88.0 & $<0.02$ & 3.3 & 3.6 & 0.13 & 0.37 & 34 & 0.52 & 0.63 & -29.10 & -5.08 & 0.70817 \\
\hline 42 & $3 / 22 / 2010$ & $<0.01$ & 165 & $<0.02$ & 2.8 & E2.0 & 0.12 & 0.48 & 31 & 0.41 & 0.70 & -24.70 & -4.09 & 0.70795 \\
\hline 46 & $8 / 13 / 2009$ & -- & 110 & -- & -- & -- & -- & -- & -- & -- & -- & -- & -- & 0.70812 \\
\hline 52 & $8 / 12 / 2009$ & -- & 236 & -- & -- & -- & -- & -- & -- & -- & -- & -- & -- & 0.70780 \\
\hline 52 & $3 / 22 / 2010$ & $<0.01$ & 191 & $<0.02$ & 2.2 & E1.8 & 0.10 & 0.33 & 31 & 0.71 & 0.56 & -25.00 & -4.19 & 0.70784 \\
\hline 61 & $3 / 22 / 2010$ & $<0.01$ & 296 & $<0.02$ & 1.8 & 3.5 & 0.07 & 0.33 & 39 & 0.55 & 0.69 & -23.60 & -3.89 & 0.70778 \\
\hline 62 & $8 / 13 / 2009$ & -- & 113 & -- & -- & -- & -- & -- & -- & -- & -- & -- & -- & 0.70814 \\
\hline 62 & $3 / 24 / 2010$ & $<0.01$ & 89.0 & $<0.02$ & 2.5 & $<2.8$ & 0.08 & 0.31 & 33 & 0.51 & 0.68 & -27.90 & -4.89 & 0.70817 \\
\hline 64 & $8 / 14 / 2009$ & -- & 158 & -- & -- & -- & -- & -- & -- & -- & -- & -- & -- & 0.70787 \\
\hline 64 & $3 / 24 / 2010$ & -- & -- & -- & -- & -- & -- & -- & -- & -- & -- & -25.30 & -4.64 & 0.70774 \\
\hline 72 & $3 / 24 / 2010$ & $<0.01$ & 336 & $<0.02$ & 1.6 & E1.6 & 0.12 & 0.22 & 45 & 0.86 & 0.82 & -22.10 & -3.89 & 0.70774 \\
\hline 75 & $8 / 17 / 2009$ & -- & 82.0 & -- & -- & -- & -- & -- & -- & -- & -- & -- & -- & 0.70855 \\
\hline 75 & $3 / 23 / 2010$ & $<0.01$ & 76.4 & E0.01 & 3.5 & 116 & 0.10 & 0.42 & 35 & 0.46 & 0.64 & -28.40 & -4.95 & 0.70879 \\
\hline 76 & $8 / 13 / 2009$ & -- & 436 & -- & -- & -- & -- & -- & -- & -- & -- & -- & -- & 0.70788 \\
\hline 77 & $8 / 13 / 2009$ & -- & 7,290 & -- & -- & -- & -- & -- & -- & -- & -- & -- & -- & 0.70799 \\
\hline
\end{tabular}

${ }^{1}$ Official U.S. Geological Survey station name not used here per landowner privacy request. 
Appendix 3. Quality-assurance water-quality data during the August 2009 and March 2010 surveys in the upper Nueces River Basin, south-central Texas.

$\left[{ }^{\circ} \mathrm{C}\right.$, degrees Celsius; mg/L, milligrams per liter; na, not applicable; EB, equipment blank sample; <, less than; FB, field blank sample; E, estimated; ENV, environmental sample; REP, sequential replicate quality-assurance sample; RPD, relative percent difference in percent; \%, percent; --, not analyzed; nc, not calculated; $\mu \mathrm{g} / \mathrm{L}$, micrograms per liter]

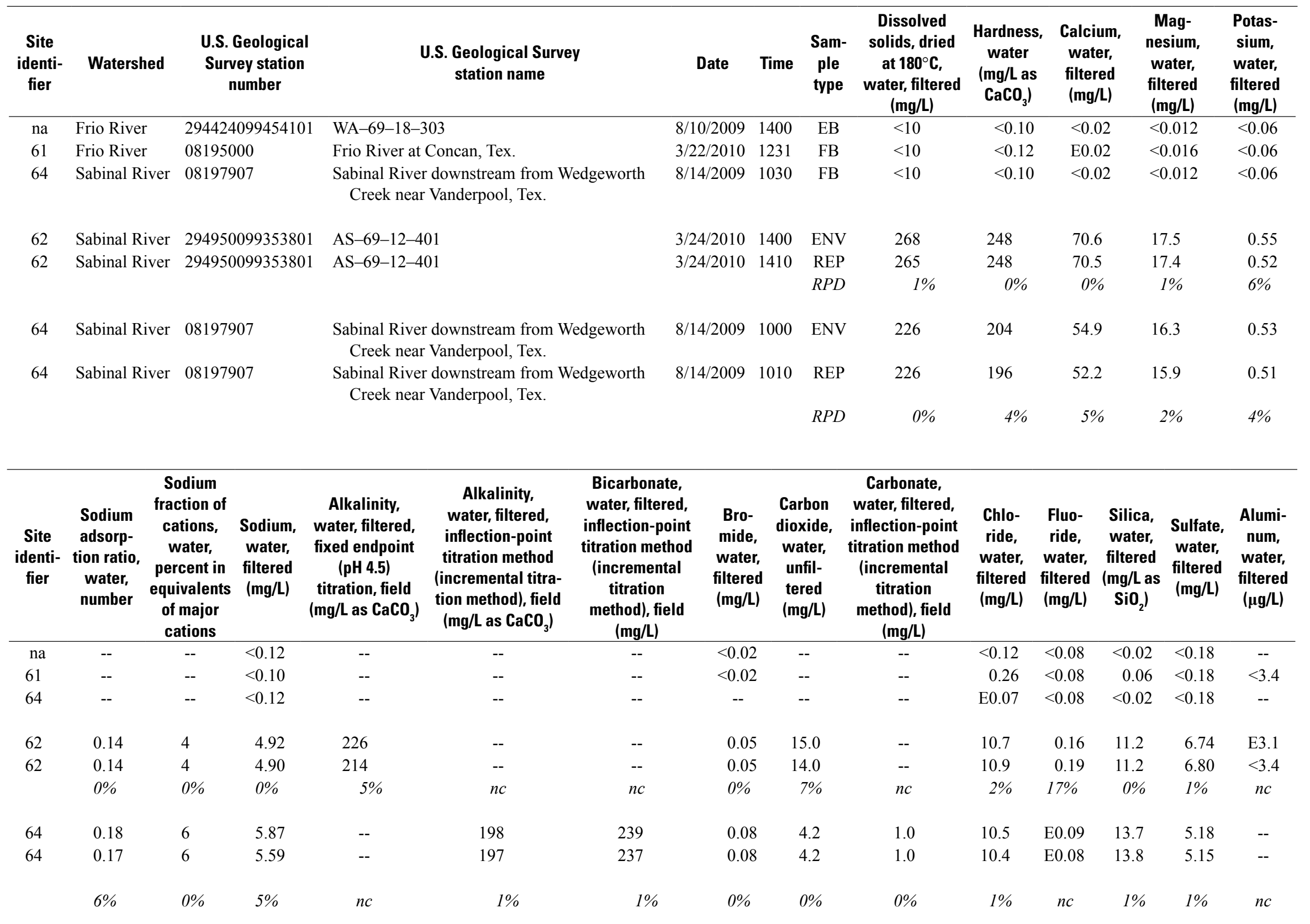


Appendix 3. Quality-assurance water-quality data during the August 2009 and March 2010 surveys in the upper Nueces River Basin, south-central Texas.-Continued

$\left[{ }^{\circ} \mathrm{C}\right.$, degrees Celsius; mg/L, milligrams per liter; na, not applicable; EB, equipment blank sample; <, less than; FB, field blank sample; E, estimated; ENV, environmental sample; REP, sequential replicate quality-assurance sample; RPD, relative percent difference in percent; \%, percent; --, not analyzed; nc, not calculated; $\mu \mathrm{g} / \mathrm{L}$, micrograms per liter]

\begin{tabular}{|c|c|c|c|c|c|c|c|c|c|c|c|c|c|}
\hline $\begin{array}{c}\text { Site } \\
\text { identifier }\end{array}$ & $\begin{array}{c}\text { Barium, } \\
\text { water } \\
\text { ( } \mu \mathrm{g} / \mathrm{L})\end{array}$ & $\begin{array}{c}\text { Beryllium, } \\
\text { water, } \\
\text { filtered } \\
(\mu \mathrm{g} / \mathrm{L})\end{array}$ & $\begin{array}{c}\text { Cadmium, } \\
\text { water, } \\
\text { filtered } \\
(\mu \mathrm{g} / \mathrm{L})\end{array}$ & $\begin{array}{l}\text { Chromium, } \\
\text { water, } \\
\text { filtered } \\
(\mu \mathrm{g} / \mathrm{L})\end{array}$ & $\begin{array}{c}\text { Cobalt, } \\
\text { water, } \\
\text { filtered } \\
(\mu \mathrm{g} / \mathrm{L})\end{array}$ & $\begin{array}{c}\text { Copper, } \\
\text { water, } \\
\text { filtered } \\
(\mu \mathrm{g} / \mathrm{L})\end{array}$ & $\begin{array}{c}\text { Iron, } \\
\text { water, } \\
\text { filtered } \\
(\mu \mathrm{g} / \mathrm{L})\end{array}$ & $\begin{array}{c}\text { Lead, } \\
\text { water, } \\
\text { filtered } \\
(\mu \mathrm{g} / \mathrm{L})\end{array}$ & $\begin{array}{c}\text { Lithium, } \\
\text { water, } \\
\text { filtered } \\
\text { ( } \mu \mathrm{g} / \mathrm{L})\end{array}$ & $\begin{array}{c}\text { Manganese, } \\
\text { water, } \\
\text { filtered } \\
(\mu \mathrm{g} / \mathrm{L})\end{array}$ & $\begin{array}{c}\text { Molybdenum, } \\
\text { water, } \\
\text { filtered } \\
(\mu \mathrm{g} / \mathrm{L})\end{array}$ & $\begin{array}{c}\text { Nickel, } \\
\text { water, } \\
\text { filtered } \\
(\mu \mathrm{g} / \mathrm{L})\end{array}$ & $\begin{array}{c}\text { Silver, } \\
\text { water, } \\
\text { filtered } \\
(\mu \mathrm{g} / \mathrm{L})\end{array}$ \\
\hline na & -- & -- & -- & -- & -- & -- & $<4$ & -- & -- & E0.2 & -- & -- & -- \\
\hline 61 & $<0.14$ & $<0.01$ & $<0.02$ & $<0.12$ & 0.51 & $<1.0$ & $<6$ & $<0.03$ & $<0.4$ & 1.0 & $<0.03$ & E0.11 & $<0.01$ \\
\hline 64 & -- & -- & -- & -- & -- & -- & $<4$ & -- & -- & $<0.2$ & -- & -- & -- \\
\hline 62 & 25 & $<0.01$ & $<0.02$ & $<0.12$ & 0.16 & $<1.0$ & $<6$ & $<0.03$ & 1.10 & E0.2 & 0.43 & 0.42 & $<0.01$ \\
\hline \multirow[t]{2}{*}{62} & 25 & $<0.01$ & $<0.02$ & $<0.12$ & 0.13 & $<1.0$ & $<6$ & $<0.03$ & 1.10 & E0.2 & 0.41 & 0.47 & $<0.01$ \\
\hline & $0 \%$ & $n c$ & $n c$ & $n c$ & $21 \%$ & $n c$ & $n c$ & $n c$ & $0 \%$ & $n c$ & $5 \%$ & $11 \%$ & $n c$ \\
\hline 64 & -- & -- & -- & -- & -- & -- & -- & -- & -- & 8.5 & -- & -- & -- \\
\hline \multirow[t]{2}{*}{64} & -- & -- & -- & -- & -- & -- & -- & -- & -- & 8.0 & -- & -- & -- \\
\hline & $n c$ & $n c$ & $n c$ & $n c$ & $n c$ & $n c$ & $n c$ & $n c$ & $n c$ & $6 \%$ & $n c$ & $n c$ & $n c$ \\
\hline $\begin{array}{c}\text { Site } \\
\text { identi- } \\
\text { fier }\end{array}$ & $\begin{array}{c}\text { Strontium, } \\
\text { water, } \\
\text { filtered } \\
\text { ( } \mu \mathrm{g} / \mathrm{L})\end{array}$ & $\begin{array}{c}\text { Thallium, } \\
\text { water, } \\
\text { filtered } \\
\text { ( } \mu \mathrm{g} / \mathrm{L})\end{array}$ & $\begin{array}{c}\text { Vanadium, } \\
\text { water, } \\
\text { filtered } \\
(\mu \mathrm{g} / \mathrm{L})\end{array}$ & $\begin{array}{c}\text { Zinc, } \\
\text { water, } \\
\text { filtered } \\
(\mu \mathrm{g} / \mathrm{L})\end{array}$ & $\begin{array}{c}\text { Antimony, } \\
\text { water, } \\
\text { filtered } \\
(\mu \mathrm{g} / \mathrm{L})\end{array}$ & $\begin{array}{c}\text { Arsenic, } \\
\text { water, } \\
\text { filtered } \\
(\mu \mathrm{g} / \mathrm{L})\end{array}$ & & $\begin{array}{c}\text { Boron, } \\
\text { water, } \\
\text { filtered } \\
(\mu \mathrm{g} / \mathrm{L})\end{array}$ & $\begin{array}{c}\text { Selenium, } \\
\text { water, } \\
\text { filtered } \\
(\mu \mathrm{g} / \mathrm{L})\end{array}$ & $\begin{array}{c}\text { Uranium } \\
\text { (natural), } \\
\text { water, } \\
\text { filtered } \\
(\mu \mathrm{g} / \mathrm{L})\end{array}$ & $\begin{array}{c}\text { Deuterium/ } \\
\text { protium } \\
\text { ratio, } \\
\text { water, } \\
\text { unfiltered } \\
\text { (per mil) }\end{array}$ & $\begin{array}{c}{ }^{18} 0 /{ }^{16} 0 \\
\text { ratio, water, } \\
\text { unfiltered } \\
\text { (per mil) }\end{array}$ & $\begin{array}{l}{ }^{87} \mathrm{Sr} /{ }^{86} \mathrm{Sr} \\
\text { ratio, water, } \\
\text { filtered } \\
\text { (dimension- } \\
\text { less) }\end{array}$ \\
\hline na & -- & -- & -- & -- & -- & -- & & -- & -- & -- & -- & -- & -- \\
\hline 61 & $<0.40$ & $<0.02$ & $<0.16$ & 12.5 & 0.06 & $<0.04$ & & $<2.8$ & $<0.04$ & $<0.01$ & -- & -- & -- \\
\hline 64 & -- & -- & -- & -- & -- & -- & & -- & -- & -- & -- & -- & -- \\
\hline 62 & 89.0 & $<0.02$ & 2.5 & $<3$ & 0.08 & 0.31 & & 33 & 0.51 & 0.68 & -27.90 & -4.89 & 0.70817 \\
\hline \multirow[t]{2}{*}{62} & 88.2 & $<0.02$ & 2.5 & E1.4 & 0.08 & 0.31 & & 32 & 0.66 & 0.68 & -27.00 & -4.90 & 0.70818 \\
\hline & $1 \%$ & $n c$ & $0 \%$ & $n c$ & $0 \%$ & $0 \%$ & & $3 \%$ & $26 \%$ & $0 \%$ & $3 \%$ & $0 \%$ & $0 \%$ \\
\hline 64 & 158 & -- & -- & -- & -- & -- & & -- & -- & -- & -- & -- & 0.70787 \\
\hline \multirow[t]{2}{*}{64} & 159 & -- & -- & -- & -- & -- & & -- & -- & -- & -- & -- & 0.70786 \\
\hline & $1 \%$ & $n c$ & $n c$ & $n c$ & $n c$ & $n c$ & & $n c$ & $n c$ & $n c$ & $n c$ & $n c$ & $0 \%$ \\
\hline
\end{tabular}


Publishing support provided by Lafayette Publishing Service Center

Information regarding water resources in Texas is available at http://tx.usgs.gov/ 
$=-\infty$
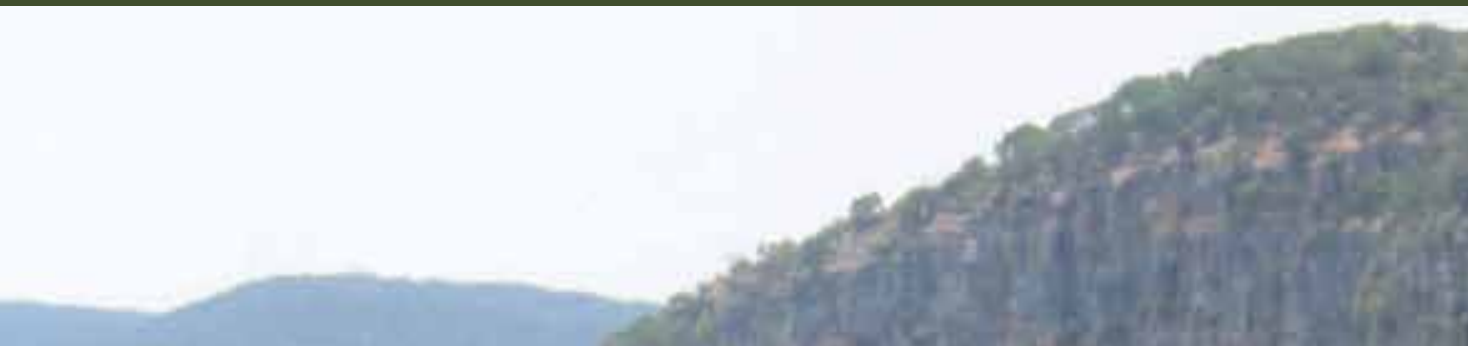

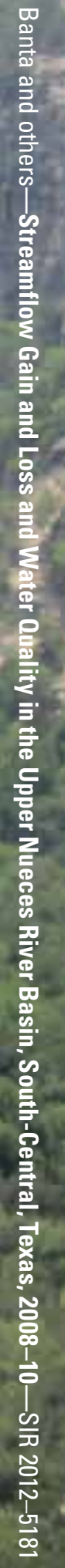

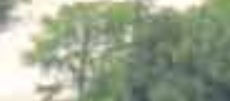

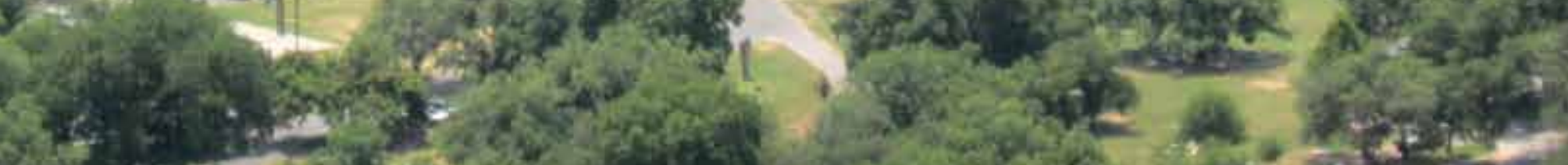

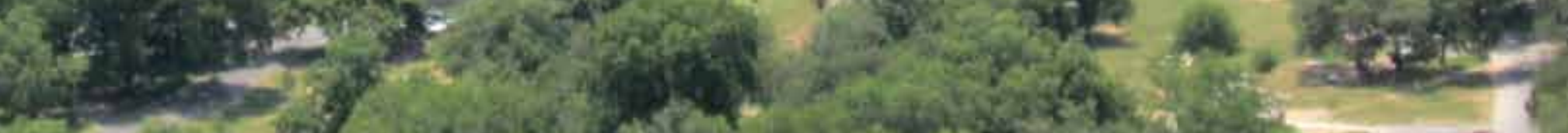
-

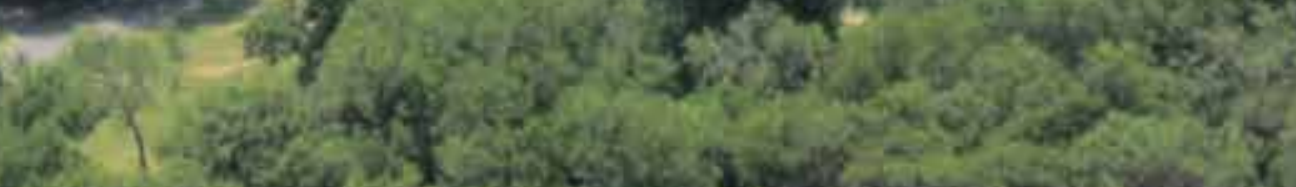

